\title{
Constitutive relations and their time integration for anisotropic elasto-plastic porous materials
}

\author{
S. Kweon ${ }^{1}$, B. Sagsoy ${ }^{1}$ and A. A. Benzerga ${ }^{2,3}$ \\ ${ }^{1}$ Mechanical Engineering, Southern Illinois University, Edwardsville, IL 62026, United States \\ ${ }^{2}$ Department of Aerospace Engineering, Texas A\&M University \\ College Station, TX 77843-3141, United States \\ ${ }^{2}$ Department of Materials Science and Engineering, Texas A\&M University \\ College Station, TX 77843-3141, United States
}

June 2, 2016

\begin{abstract}
Finite deformation constitutive relations are developed for a class of plastically anisotropic porous solids with an underlying evolving microstructure. They are based on a model obtained by homogenization for rigid-perfectly plastic materials containing non-spherical voids. To facilitate numerical implementation, heuristic extensions are proposed to incorporate weak elasticity, strain hardening and accurate void shape evolution. A semi-implicit time integration scheme is used along with the NewtonRaphson method to solve the system of equations resulting from the discretization of the constitutive equations. The procedure to calculate the consistent tangent matrix, which is needed to solve the global force-displacement matrix equation is summarized. The framework is used to illustrate the predictive capabilities of the model, first under conditions previously assessed against finite element cell model calculations, then under conditions heretofore not examined. The latter include situations of initial anisotropy as well as situations involving significant void distortions, not only in terms of void enlargement or shape change, but also in terms of void rotations. In particular, various combinations of stress triaxiality, initial void shape, void orientation, matrix orthotropy properties and loading directions are simulated. In addition, the finite element implementation of the model is addressed and illustrated for simple cases.
\end{abstract}

Keywords: plastic anisotropy; microstructural spin; consistent tangent; finite elements; ductile fracture

\section{Introduction}

The damage mechanics of ductile materials has been widely studied both experimentally and computationally. The most widely used model of ductile damage was developed by Gurson [1] who derived a plastic yield function through the homogenization of a representative 
volume element in the form of a spherical shell. The model has been improved for computational purposes by Tvergaard and Needleman [2] and further extensions have been proposed since [3]. Other ductile damage models have been developed, e.g. within the framework of continuum thermomechanics [4-6] (see [7] for a review) or homogenization [8-11] and used in various simulations of damage and metal forming processes [12-14].

Recent progress has been either on computational methods, often implemented with relatively simple constitutive relations, or on micromechanical analyses often aimed at assessing advanced constitutive relations. An example of the first type of investigations concerns the development of nonlocal formulations suitable for numerical analysis. Because the various damage mechanisms represented in the available models cause softening, they eventually lead to ill-posedness of the incremental problem. One manifestation of the latter is the pathological mesh dependence in the computed solution. To address this, weakly nonlocal models have been introduced in simulations of ductile damage [15-17]. The mesh sensitivity is prevented under certain circumstances using an integral condition on the increment of the damage quantity $[11,16,18]$. Furthemore, gradient enhanced models were developed to suppress mesh-sensitive localization [19,20]. Similarly, a strain-gradient based model was used by Lorentz et al. [21] who also employed a mixed finite element formulation to avoid volumetric locking in near-incompressible plastic flow. More recently, a second-gradient model that possesses the same number of degrees of freedom per node as those of first-gradient models was proposed and used to remove mesh sensitivity and improve convergence [20]. Ductile damage accumulation eventually causes materials to develop cracks and fracture. In order to seamlessly represent damage to cracking transitions, attempts have been made to integrate the computational scheme for crack tip discontinuity and ductile damage formulations. Crack propagation can thus be simulated by combining the GTN (Gurson-Tvergaard-Needleman) model [2] and extended finite element method [22]. Another approach in this regard is to combine ductile damage and a cohesive zone model to alleviate difficulties associated with crack tip discontinuity. A variant of this consists of using a finite-thickness band (nonlocal type) formulation [23].

Another direction in ductile damage investigations has focused on the anisotropy in damage evolution. Physically, the anisotropy can be rooted in that of the undamaged material (referred to here as plastic anisotropy) or manifests through the evolution of the shape and orientation of voids and microcracks; this type of anisotropy is referred to as morphological. Both the plastic and morphological anisotropies can be initial or induced, although the former is generally initial and the latter essentially induced. There is a great deal of interest in developing accurate yield functions for anisotropic materials in the absence of damage [24-26]. The use of advanced anisotropic yield functions may have significant implications when ductile fracture follows from the onset of plastic instabilities [27]. However, these models did not set out to incorporate the effects of damage, except in uncoupled ways [28]. In some formulations based on continuum thermomechanics, the damage variable (with an appropriate tensorial representation as discussed in [29]) is taken to evolve dependent upon stress (or strain) directions [30,31]. One advantage of this approach is the relative simplicity of the constitutive relations, including for damage evolution. On the other hand, they are generally semi-coupled in that the influence of field variables describing damage and plastic flow enter two separate thermodynamic potentials. By way of contrast, the coupling between damage and plasticity is seamlessly realized in the homogenization based models $[8,10,32-37]$ that 
are built on earlier isotropic formulations [1,2]. In particular, fully coupled models that account for both plastic and morphological anisotropies were independently developed by Keralavarma and Benzerga [38,39] and Monchiet et al. [40] who considered rigid-perfectly plastic materials containing spheroidal voids. In addition, in [39] evolution equations were developed for the internal variables. The constitutive relations and associated evolution equations for damage related entities are quite complex owing to the mathematical nature of scale transitions.

While computational methods for implementing isotropic dilatant plasticity models are now well established, e.g., $[7,17,41]$ little is available on anisotropic models where the source of anisotropy is microstructural and hence evolving. A particularly challenging problem consists of formulating robust, accurate and fast-converging methods for dealing with microstructural spins. Benzerga et al. [42,43] first proposed a finite-element implementation of homogenization-based anisotropic porous metal plasticity. In their formulation, the voids were taken to rotate with the material, following [32,34]. Aravas and Ponte Castaneda [44] proposed an alternative method which accounts for the change in the orientation of axes for ellipsoidal cavities. They used explicit integration on several internal state variables, including the void orientation. Here, an efficient semi-implicit time-integration algorithm for dealing with finite-element implementation of anisotropic porous metal plasticity is proposed, which enables in principle large time-step computations. Heuristic extensions of the model in [39] are proposed to incorporate weak elasticity and accurate void shape evolution, and enable computational analyses of boundary-value problems, which are still lacking for this class of models. The complete constitutive model is presented in Section 2. The time integration scheme and the finite-element implementation are provided in detail in Section 3. In Section 4, simulation results are compiled which cover various combinations of void shapes and orientations, plastic anisotropy, and loading directions. The notation used in this paper is as follows. First order tensors, i.e. vectors, are denoted using a bar underneath. Second and fourth order tensors are denoted by bold and double-stroke letters, respectively. For example, $\mathbb{I}$ and $\boldsymbol{I}$ are the fourth and second order identity tensors, respectively. Tensor operations are defined as follows, $\boldsymbol{A} \underline{b}=A_{i j} b_{j}, \boldsymbol{A} \boldsymbol{B}=A_{i j} B_{j k}, \boldsymbol{A}: \boldsymbol{B}=A_{i j} B_{i j}$, and $(\underline{a} \otimes \underline{b})_{i j}=a_{i} b_{j}$ for the dyadic product, with the summation convention used over repeated indices. The roman subscript $\mathrm{m}$ indicates the average of the diagonal components of a second order tensor: $A_{\mathrm{m}} \equiv A_{k k} / 3$.

\section{Constitutive Relations}

The constitutive framework is that for a porous plastic solid made up of a plastically anisotropic matrix containing aligned nonspherical voids with arbitrary initial orientations [39]; see Fig. 1. To enable numerical implementation and application to engineering materials, their formulation is extended as follows ${ }^{1}$. The rate of deformation tensor $\boldsymbol{D}$ is additively decomposed into:

$$
\boldsymbol{D}=\boldsymbol{D}^{\mathrm{e}}+\boldsymbol{D}^{\mathrm{p}}
$$

\footnotetext{
${ }^{1}$ The model may be implemented following various strategies within either a hypoelastic or hyperelastic formulation. The choice made here is set by the structure of the user-defined routine of the commercial code Abaqus.
} 
where the elastic part is given by:

$$
D^{\mathrm{e}}=\mathbb{L}^{-1}: \stackrel{\nabla}{\boldsymbol{\sigma}}
$$

with $\mathbb{L}$ the isotropic tensor of elastic moduli and $\stackrel{\nabla}{\boldsymbol{\sigma}}$ the Jaumann stress rate defined by:

$$
\stackrel{\nabla}{\sigma}=\dot{\sigma}+\sigma \Omega-\Omega \sigma
$$

where $\boldsymbol{\Omega}$ is the skew symmetric part of the velocity gradient. The plastic part of $\boldsymbol{D}$ is given by normality to a yield surface $^{2} \mathcal{F}(\boldsymbol{\sigma})=0$

$$
\boldsymbol{D}^{\mathrm{p}}=\Lambda \frac{\partial \mathcal{F}}{\partial \boldsymbol{\sigma}}(\boldsymbol{\sigma})
$$

where $\Lambda$ is the plastic multiplier and

$$
\mathcal{F}(\boldsymbol{\sigma})=C \frac{\sigma_{\text {eq }}^{2}}{\bar{\sigma}^{2}}+2(g+1)(g+f) \cosh \left(\kappa \frac{\boldsymbol{\sigma}: \boldsymbol{X}}{\bar{\sigma}}\right)-(g+1)^{2}-(g+f)^{2}
$$

where $\sigma_{\text {eq }}$ is an equivalent stress that could be based on advanced anisotropic plasticity in the absence of porosity $[26,36]$. In the original model [39], yielding in the matrix is taken to obey Hill's criterion [45] and $\sigma_{\text {eq }}$ is defined as:

$$
\sigma_{\mathrm{eq}}^{2}=\frac{3}{2} \boldsymbol{\sigma}: \mathbb{H}: \boldsymbol{\sigma}
$$

where $\mathbb{H}$ is related to Hill's anisotropy tensor $\mathbb{p}$ through:

$$
\mathbb{H} \equiv \mathbb{p}+\eta(\boldsymbol{X} \otimes \boldsymbol{Q}+\boldsymbol{Q} \otimes \boldsymbol{X}), \quad \mathbb{p} \equiv \mathbb{J}: \mathbb{h}: \mathbb{J}, \quad \mathbb{J} \equiv \mathbb{I}-\frac{1}{3} \boldsymbol{I} \otimes \boldsymbol{I}
$$

Here, $\boldsymbol{Q}$ is a constant tensor and $\boldsymbol{X}$ a void-shape dependent tensor, which are both transversely isotropic in the frame associated with the void (Fig. 1), and are given by:

$$
\begin{gathered}
\boldsymbol{Q} \equiv-\frac{1}{2}\left(\underline{n}_{1} \otimes \underline{n}_{1}+\underline{n}_{2} \otimes \underline{n}_{2}\right)+\underline{n}_{3} \otimes \underline{n}_{3}, \\
\boldsymbol{X} \equiv \alpha_{2}\left(\underline{n}_{1} \otimes \underline{n}_{1}+\underline{n}_{2} \otimes \underline{n}_{2}\right)+\left(1-2 \alpha_{2}\right) \underline{n}_{3} \otimes \underline{n}_{3}
\end{gathered}
$$

where $\underline{n}_{3}$ is the void axis and $\underline{n}_{1}$ and $\underline{n}_{2}$ are orthogonal base vectors arbitrarily chosen in the transverse plane. Also, $\bar{\sigma}$ in $(5)$ is the flow strength of the matrix material in a reference direction. In practice, $\bar{\sigma}$ is selected as the yield strength in one of the principal directions of orthotropy and the components of the anisotropy tensor $\mathbb{p}$ are scaled accordingly. In addition, $f$ denotes the porosity and the criterion parameters $C, g$ and $\kappa$ in (5) as well as $\eta$ in (7) and $\alpha_{2}$ in (9) are functions of $f$ and eventually the void aspect ratio $w$ and the components of $\mathbb{p}$. Their expressions are provided in Appendix A. In (7) $\mathbb{I}$ denotes the deviatoric projection operator, i.e., $J_{i j k l}=\frac{1}{2}\left(\delta_{i k} \delta_{j l}+\delta_{i l} \delta_{j k}\right)-\frac{1}{3} \delta_{i j} \delta_{k l}$, and $\mathbb{h}$ denotes the anisotropy tensor in the space of deviatoric stresses.

Microstructural changes are specified in terms of evolution equations of the porosity, $f$, the void aspect ratio, $w$, and the void axis, $\underline{n}_{3}$, which constitute the internal state variables.

\footnotetext{
${ }^{2}$ In a rate dependent formulation, $\mathcal{F}(\boldsymbol{\sigma})=0$ may be used as a gauge surface as in [2] and [43].
} 
The orientation of the principal axes of orthotropy, denoted by $\underline{e}_{L}, \underline{e}_{T}$ and $\underline{e}_{S}$ (Fig. 1), is taken to rotate with the material. Note that the current matrix anisotropy basis and the basis tied to the voids do not necessarily coincide, even when they do initially. Also, in general the principal loading directions are not aligned with either $\left(\underline{e}_{L}, \underline{e}_{T}, \underline{e}_{S}\right)$ or $\left(\underline{n}_{1}, \underline{n}_{2}, \underline{n}_{3}\right)$; see Fig. 1. The evolution of porosity is governed by plastic incompressibility such that:

$$
\dot{f}=(1-f) D_{k k}^{\mathrm{p}}=(1-f) \Lambda \frac{\partial \mathcal{F}}{\partial \sigma_{\mathrm{m}}}
$$

with due account of the hydrostatic part in the flow rule (4). The void aspect ratio is defined such that $w>1$ corresponds to prolate voids. It is evolved according to (with $S \equiv \ln (w))$ :

$$
\dot{S}=\boldsymbol{Q}:\left[\left(1+k_{\mathrm{w}} k_{\mathrm{f}} k_{\mathcal{T}}\right) \boldsymbol{D}^{\mathrm{p}}+3\left(\frac{1}{f} \boldsymbol{X}^{v}-\boldsymbol{X}\right) D_{\mathrm{m}}^{\mathrm{p}}\right]
$$

where $\boldsymbol{X}^{v}$ is defined similar to $\boldsymbol{X}$ in (9) with $\alpha_{2}$ replaced by $\alpha_{1}$, a function of $f$ and $w$ defined in Appendix A. In the original model [39] $k_{\mathrm{e}}=k_{\mathrm{f}}=k_{\mathcal{T}}=0$ but here, following [34], they are given by

$$
\begin{aligned}
& k_{\mathrm{w}}(w)=\frac{9}{2} \frac{\alpha_{1}-\alpha_{1}^{\mathrm{Gar}}}{1-3 \alpha_{1}}, \\
& k_{\mathrm{f}}(f)=(1-\sqrt{f})^{2} \\
& k_{\mathcal{T}}(\mathcal{T}, \epsilon)=\left\{\begin{array}{l}
1-\frac{\mathcal{T}^{2}+\mathcal{T}^{4}}{9} \text { for } \quad \epsilon=+1 \\
1-\frac{\mathcal{T}^{2}+\mathcal{T}^{4}}{18} \text { for } \quad \epsilon=-1
\end{array}, \quad \epsilon \equiv \operatorname{sgn}\left(\sigma_{\mathrm{m}} \sigma_{33}^{\prime}\right)\right. \\
& \mathcal{T}=\frac{\sigma_{k k} / 3}{\sqrt{\frac{3}{2} \boldsymbol{\sigma}^{\prime}: \boldsymbol{\sigma}^{\prime}}}
\end{aligned}
$$

where $\mathcal{T}$ is the stress triaxiality and $\epsilon$ is related to the third stress invariant; also see [43]. In the above equations the function $\alpha_{1}^{\mathrm{Gar}}(f, w)$ is given in Appendix A. Heuristic functions $k_{\mathrm{w}}, k_{\mathrm{f}}$ and $k_{\mathcal{T}}$ correct the mismatch due to void shape, porosity and stress triaxiality, respectively, between unit cell simulation results and model predictions. We shall use the shorthand notation $k=1+k_{\mathrm{w}} k_{\mathrm{f}} k_{\mathcal{T}}$ wherever appropriate. Note that the evolution equation of $w$ includes an implicit dependence upon matrix anisotropy through the macroscopic rate of plastic deformation, $\boldsymbol{D}^{\mathrm{p}}$, which is derived from the yield criterion (5) by normality.

The evolution of void orientation is obtained using the following kinematical relation

$$
\underline{\dot{n}}_{3}=\boldsymbol{\omega} \cdot \underline{n}_{3}, \quad \boldsymbol{\omega}=\boldsymbol{\Omega}^{v}+\boldsymbol{\Omega}^{l}
$$

where the rotation tensor $\boldsymbol{\omega}$ accounts for the macroscopic spin of the material and the local plastic distortion following Kailasam and Ponte Castaneda [46]. Specifically, the void spin tensor $\Omega^{v}$ is related to the continuum spin tensor $\Omega$ via:

$$
\Omega^{v}=\Omega-\mathbb{C}: D^{\mathrm{p}}
$$

where $\mathbb{C}$ is the fourth order spin concentration tensor given by

$$
\mathbb{C}=-(1-f) \mathbb{P}: \mathbb{A}, \quad \mathbb{A}=[\mathbb{I}-(1-f) \mathbb{S}]^{-1}
$$


where $\mathbb{A}$ is the strain concentration tensor and $\mathbb{P}$ and $\mathbb{S}$ are the Eshelby tensors [47] for a spheroidal inclusion of zero stiffness in an incompressible linear viscous matrix. Also, in (16) $\Omega^{l}$ is an additional contribution to the effective void rotation that comes from mere distortion of void boundaries. The components of this antisymmetric tensor in the frame tied to the voids are given by:

$$
\Omega_{12}^{l}=0, \quad \Omega_{i 3}^{l}=\frac{w^{2}+1}{w^{2}-1} D_{i 3}^{v} \quad(i=1,2, \quad w \neq 1)
$$

with $\boldsymbol{D}^{v}$ representing the distortion of the void, which in [33] is related to the plastic rate of deformation through

$$
\boldsymbol{D}^{v}=\mathbb{A}: \boldsymbol{D}^{\mathrm{p}}
$$

where the concentration tensor $\mathbb{A}$ is given by (18) 2 . Since $\mathbb{A}$ is based on Eshelby's tensor, it inevitably leads to inaccurate predictions in the nonlinear case [48]. The following form is therefore preferred:

$$
\boldsymbol{D}^{v}=k \boldsymbol{D}^{\mathrm{p}}+3\left(\frac{1}{f} \boldsymbol{X}^{v}-\boldsymbol{X}\right) D_{\mathrm{m}}^{\mathrm{p}}
$$

which underlies the evolution of void shape, equation (11), with the provision of the heuristic function $k$. Defining the plastic spin tensor as $\Omega^{\mathrm{p}}=\boldsymbol{\Omega}-\boldsymbol{\omega}$ one obtains [46]

$$
\boldsymbol{\Omega}^{\mathrm{p}}=\mathbb{C}: \boldsymbol{D}^{\mathrm{p}}-\frac{1}{2} \sum_{i \neq j, w_{i} \neq w_{j}} \frac{w_{i}^{2}+w_{j}^{2}}{w_{i}^{2}-w_{j}^{2}}\left[\left(\underline{n}_{i} \otimes \underline{n}_{j}+\underline{n}_{j} \otimes \underline{n}_{i}\right): \mathbb{A}: \boldsymbol{D}^{\mathrm{p}}\right] \underline{n}_{i} \otimes \underline{n}_{j}
$$

by combining Eq. (17) and (16) $)_{2}$ and adopting the notation $w_{1}=w_{2}=w$ and $w_{3}=1$. Writing the above equation in direct form follows the formulation in [44]. It enables straighforward evaluation of the components of $\boldsymbol{\omega}$ with respect to a laboratory frame. The case $w=1$ requires a careful treatment, see Section 3.1. It is worth mentioning that more recent developments within an "iterative" approach [49] alleviate some of the inaccuracies of estimate (20). No attempt has been made here to compare heuristics (21) with such new estimates. Also, as noted in [39], the concentration tensors used in (17)-(20) are in all rigor limited to isotropic matrices. Only numerical estimates are available for anisotropic matrices [50]. Nevertheless, as in Eq. (11) for the void shape evolution, Eq. (17) includes an implicit (approximate) dependence of $\Omega^{v}$ on material anisotropy through the macroscopic rate of deformation.

Finally, isotropic strain hardening of the matrix material is incorporated as in the GTN model. The effective stress $\bar{\sigma}$ is taken to depend on some effective plastic strain $\bar{\epsilon}$. Here, a power law of the form:

$$
\bar{\sigma}=\sigma_{\mathrm{S}}\left(1+\bar{\epsilon} / \epsilon_{0}\right)^{N}
$$

is used. The evolution of $\bar{\epsilon}$ is obtained considering the plastic work equivalence between the macroscopic homogeneous material and the matrix:

$$
\boldsymbol{\sigma}: \boldsymbol{D}^{\mathrm{p}}=(1-f) \bar{\sigma} \dot{\bar{\epsilon}}
$$




\section{Numerical Implementation}

\subsection{Corotational Formulation}

The anisotropic ductile damage model presented in the previous section is formulated within a corotational framework [51]. The constitutive relations are expressed in an intermediate configuration, which is rotated from the current one by $\boldsymbol{R}^{*}$, the rotation tensor that results from the polar decomposition of the incremental deformation gradient:

$$
\Delta \boldsymbol{F}=\boldsymbol{R}^{*} \boldsymbol{U}^{*}
$$

Incremental objectivity is preserved by using the Jaumann rate (3) in (2). Quantities defined in the rotated configuration are indicated by the tilde $\left(^{\sim}\right)$ symbol. Constitutive descriptions need to be written only for the stretch part of deformations since the rotation part is taken care of by rotating quantities to the intermediate configuration. Standard transformation rules apply, e.g., $\tilde{\boldsymbol{A}}=\boldsymbol{R}^{* T} \boldsymbol{A} \boldsymbol{R}^{*}$. The constitutive equations are then rewritten in the rotated configuration. For example, the hypoelastic law, which is written in the current configuration in Eq. (2), is converted to

$$
\tilde{\boldsymbol{D}}^{\mathrm{e}}=\mathbb{L}^{-1}: \dot{\tilde{\boldsymbol{\sigma}}}
$$

in the rotated configuration. Note that $\tilde{\mathbb{L}}=\mathbb{L}$ because of the assumed isotropic elasticity. The objective rate $\stackrel{\boldsymbol{\sigma}}{\text { is }}$ replaced by $\dot{\tilde{\boldsymbol{\sigma}}}$, which is the main advantage of utilizing the corotational formulation. In other words, the constitutive updating is done without calculating the objective rate. The yield criterion, eq. (5), is simply re-written as $\mathcal{F}(\tilde{\boldsymbol{\sigma}})=0$ with tensors $\mathbb{H}$, $\boldsymbol{X}$ and $\boldsymbol{Q}$ replaced with $\tilde{\mathbb{H}}, \tilde{\boldsymbol{X}}$ and $\tilde{\boldsymbol{Q}}$, respectively. The flow rule (4) corresponding to yield criterion (5) writes:

$$
\tilde{\boldsymbol{D}}^{\mathrm{p}}=\Lambda\left[3 C \frac{\tilde{\mathbb{H}}: \tilde{\boldsymbol{\sigma}}}{\bar{\sigma}^{2}}+2(g+1)(g+f) \kappa \sinh \left(\kappa \frac{\tilde{\boldsymbol{\sigma}}: \tilde{\boldsymbol{X}}}{\bar{\sigma}}\right) \frac{\tilde{\boldsymbol{X}}}{\bar{\sigma}}\right]
$$

Combining (26) and (27) the additive decomposition (1) leads to:

$$
\mathbb{L}^{-1}: \dot{\tilde{\boldsymbol{\sigma}}}+\Lambda\left[3 C \frac{\tilde{\mathbb{H}}: \tilde{\boldsymbol{\sigma}}}{\bar{\sigma}^{2}}+2(g+1)(g+f) \kappa \sinh \left(\kappa \frac{\tilde{\boldsymbol{\sigma}}: \tilde{\boldsymbol{X}}}{\bar{\sigma}}\right) \frac{\tilde{\boldsymbol{X}}}{\bar{\sigma}}\right]=\tilde{\boldsymbol{D}}
$$

Using (27) again, the equations (10) and (11), respectively giving the evolution of the void volume fraction and void shape, are recast into

$$
\dot{f}=(1-f) \Lambda\left[3 C \frac{\boldsymbol{I}: \tilde{\mathbb{H}}: \tilde{\boldsymbol{\sigma}}}{\bar{\sigma}^{2}}+2(g+1)(g+f) \kappa \sinh \left(\kappa \frac{\tilde{\boldsymbol{\sigma}}: \tilde{\boldsymbol{X}}}{\bar{\sigma}}\right) \frac{\boldsymbol{I}: \tilde{\boldsymbol{X}}}{\bar{\sigma}}\right]
$$

and

$$
\begin{aligned}
\dot{S}= & \Lambda k \tilde{\boldsymbol{Q}}:\left[3 C \frac{\tilde{\mathbb{H}}: \tilde{\boldsymbol{\sigma}}}{\bar{\sigma}^{2}}+2(g+1)(g+f) \kappa \sinh \left(\kappa \frac{\tilde{\boldsymbol{\sigma}}: \tilde{\boldsymbol{X}}}{\bar{\sigma}}\right) \frac{\tilde{\boldsymbol{X}}}{\bar{\sigma}}\right] \\
& +\Lambda \tilde{\boldsymbol{Q}}:\left(\frac{1}{f} \tilde{\boldsymbol{X}}^{v}-\tilde{\boldsymbol{X}}\right)\left[3 C \frac{\boldsymbol{I}: \tilde{\mathbb{H}}: \tilde{\boldsymbol{\sigma}}}{\bar{\sigma}^{2}}+2(g+1)(g+f) \kappa \sinh \left(\kappa \frac{\tilde{\boldsymbol{\sigma}}: \tilde{\boldsymbol{X}}}{\bar{\sigma}}\right) \frac{\boldsymbol{I}: \tilde{\boldsymbol{X}}}{\bar{\sigma}}\right]
\end{aligned}
$$


and that giving the evolution of the matrix plastic strain, eq. (24), into

$$
\dot{\bar{\epsilon}}=\frac{1}{1-f} \Lambda\left[3 C \frac{\tilde{\boldsymbol{\sigma}}: \tilde{\mathbb{H}}: \tilde{\boldsymbol{\sigma}}}{\bar{\sigma}^{3}}+2(g+1)(g+f) \kappa \sinh \left(\kappa \frac{\tilde{\boldsymbol{\sigma}}: \tilde{\boldsymbol{X}}}{\bar{\sigma}}\right) \frac{\tilde{\boldsymbol{\sigma}}: \tilde{\boldsymbol{X}}}{\bar{\sigma}^{2}}\right]
$$

The equation for the evolution of void orientation, (16), is transformed into

$$
\underline{\tilde{\tilde{n}}}_{3}=-\tilde{\Omega}^{\mathrm{p}} \underline{\tilde{n}}_{3}
$$

where $-\tilde{\boldsymbol{\Omega}}^{p}$ formally replaces $\boldsymbol{\omega}$ of (16) due to the kinematical relationship between the rotated and current configurations. Based on Eq. (22), $\tilde{\Omega}^{\mathrm{p}}$ is given by

$$
\tilde{\boldsymbol{\Omega}}^{\mathrm{p}}=\boldsymbol{R}^{* T} \boldsymbol{\Omega}^{\mathrm{p}} \boldsymbol{R}^{*}=\tilde{\mathbb{C}}: \tilde{\boldsymbol{D}}^{\mathrm{p}}-\frac{1}{2} \sum_{i \neq j, w_{i} \neq w_{j}} \frac{w_{i}^{2}+w_{j}^{2}}{w_{i}^{2}-w_{j}^{2}}\left(\underline{\tilde{n}}_{i} \otimes \underline{\tilde{n}}_{j}+\underline{\tilde{n}}_{j} \otimes \underline{\tilde{n}}_{i}\right): \tilde{\mathbb{A}}: \tilde{\boldsymbol{D}}^{\mathrm{p}} \underline{\tilde{n}}_{i} \otimes \underline{\tilde{n}}_{j}
$$

For a spherical void $(w=1)$, the void axis $\underline{n}_{3}$ is a priori ill-defined. It is determined as the direction of maximum "stretching of the void", i.e. based on the diagonalization of the void distortion tensor $\boldsymbol{D}^{v}$ given by (21) above. This is clearly an approximation since for general strain states, the void shape actually becomes ellipsoidal $[10,46,48]$. More generally, passage through $w=1$ requires additional care in evaluating functions $k_{\mathrm{w}}$ (Eq.(12)), $e_{2}$ (Eq.(A.2) 2 ), $C$ (Eq.(A.8) $)_{2}$ ) and $\alpha_{1}$ (Eq.(A.10)). All these functions admit finite asymptotic values in the limit $w \rightarrow 0$. In the computations, whenever the void aspect ratio is close to 1 , say $0.99 \leq w \leq 1.01$, the above functions are replaced with their asymptotic values, see e.g., [3]

\subsection{Time Integration Method}

To integrate the above constitutive equations, a semi-implicit integration algorithm is employed. The state variables in the rotated configuration are assembled in the vector set

$$
[\boldsymbol{V}]^{T}=\left[\tilde{\boldsymbol{\sigma}}^{\prime}, \tilde{\sigma}_{\mathrm{m}}, f, S, \bar{\epsilon}, \Lambda\right]
$$

where the deviatoric-volumetric decomposition [52] is applied to the Cauchy stress $\boldsymbol{\sigma}$ to facilitate the convergence in the Newton-Raphson procedure employed below. The void orientation, $\underline{n}_{3}$, is an additional state variable for which an explicit update is justified below, hence the semi-implicit character of the proposed scheme. The (differential) equations for the above variables are (28), (29), (30), (31), (23), and (5), respectively. This coupled system is solved using an implicit method following a backward Euler scheme. The residuals are collected in the following vector set

$$
[\boldsymbol{R}]^{T}=\left[\boldsymbol{R}_{\tilde{\boldsymbol{\sigma}}^{\prime}}, R_{\tilde{\sigma}_{\mathrm{m}}}, R_{f}, R_{S}, R_{\bar{\epsilon}}, R_{\Lambda}\right] .
$$

and their expressions are as follows:

$$
\begin{aligned}
\boldsymbol{R}_{\tilde{\boldsymbol{\sigma}}^{\prime}}= & \frac{1}{2 \mu}\left(\frac{\tilde{\boldsymbol{\sigma}}^{\prime}-\tilde{\boldsymbol{\sigma}}_{0}^{\prime}}{\Delta t}\right) \\
& +\Lambda\left[3 C \frac{\mathbb{\mathbb { J }}: \tilde{\mathbb{H}}: \tilde{\boldsymbol{\sigma}}}{\bar{\sigma}^{2}}+2(g+1)(g+f) \kappa \sinh \left(\kappa \frac{\tilde{\boldsymbol{\sigma}}: \tilde{\boldsymbol{X}}}{\bar{\sigma}}\right) \frac{\mathbb{J}: \tilde{\boldsymbol{X}}}{\bar{\sigma}}\right]-\tilde{\boldsymbol{D}}^{\prime}
\end{aligned}
$$




$$
\begin{aligned}
& R_{\tilde{\sigma}_{\mathrm{m}}}=\frac{1}{K \Delta t}\left[\tilde{\sigma}_{\mathrm{m}}-\left(\tilde{\sigma}_{\mathrm{m}}\right)_{0}\right] \\
& +\Lambda\left[3 C \frac{\boldsymbol{I}: \tilde{\mathbb{H}}: \tilde{\boldsymbol{\sigma}}}{\bar{\sigma}^{2}}+2(g+1)(g+f) \kappa \sinh \left(\kappa \frac{\tilde{\boldsymbol{\sigma}}: \tilde{\boldsymbol{X}}}{\bar{\sigma}}\right) \frac{\boldsymbol{I}: \tilde{\boldsymbol{X}}}{\bar{\sigma}}\right]-\tilde{D}_{k k} \\
& R_{f}=\frac{f-f_{0}}{\Delta t} \\
& -(1-f) \Lambda\left[3 C \frac{\boldsymbol{I}: \tilde{\mathbb{H}}: \tilde{\boldsymbol{\sigma}}}{\bar{\sigma}^{2}}+2(g+1)(g+f) \kappa \sinh \left(\kappa \frac{\tilde{\boldsymbol{\sigma}}: \tilde{\boldsymbol{X}}}{\bar{\sigma}}\right) \frac{\boldsymbol{I}: \tilde{\boldsymbol{X}}}{\bar{\sigma}}\right] \\
& R_{S}=\frac{S-S_{0}}{\Delta t}-\Lambda k \tilde{\boldsymbol{Q}}:\left[3 C \frac{\tilde{\mathbb{H}}: \tilde{\boldsymbol{\sigma}}}{\bar{\sigma}^{2}}+2(g+1)(g+f) \kappa \sinh \left(\kappa \frac{\tilde{\boldsymbol{\sigma}}: \tilde{\boldsymbol{X}}}{\bar{\sigma}}\right) \frac{\tilde{\boldsymbol{X}}}{\bar{\sigma}}\right] \\
& -\Lambda \tilde{\boldsymbol{Q}}:\left(\frac{1}{f} \tilde{\boldsymbol{X}}^{v}-\tilde{\boldsymbol{X}}\right)\left[3 C \frac{\boldsymbol{I}: \tilde{\mathbb{H}}: \tilde{\boldsymbol{\sigma}}}{\bar{\sigma}^{2}}+2(g+1)(g+f) \kappa \sinh \left(\kappa \frac{\tilde{\boldsymbol{\sigma}}: \tilde{\boldsymbol{X}}}{\bar{\sigma}}\right) \frac{\boldsymbol{I}: \tilde{\boldsymbol{X}}}{\bar{\sigma}}\right] \\
& R_{\bar{\epsilon}}=\frac{\bar{\epsilon}-\bar{\epsilon}_{0}}{\Delta t} \\
& -\frac{1}{1-f} \Lambda\left[3 C \frac{\tilde{\boldsymbol{\sigma}}: \tilde{\mathbb{H}}: \tilde{\boldsymbol{\sigma}}}{\bar{\sigma}^{3}}+2(g+1)(g+f) \kappa \sinh \left(\kappa \frac{\tilde{\boldsymbol{\sigma}}: \tilde{\boldsymbol{X}}}{\bar{\sigma}}\right) \frac{\tilde{\boldsymbol{\sigma}}: \tilde{\boldsymbol{X}}}{\bar{\sigma}^{2}}\right] \\
& R_{\Lambda}=C \frac{3}{2} \frac{\tilde{\boldsymbol{\sigma}}: \tilde{\mathbb{H}}: \tilde{\boldsymbol{\sigma}}}{\bar{\sigma}^{2}}+2(g+1)(g+f) \cosh \left(\kappa \frac{\tilde{\boldsymbol{\sigma}}: \tilde{\boldsymbol{X}}}{\bar{\sigma}}\right)-(g+1)^{2}-(g+f)^{2}
\end{aligned}
$$

where $\boldsymbol{V}_{0}$ and $\boldsymbol{V}$ respectively denote the state variables at the beginning and at the end of the increment $\Delta t$. Also, $K$ and $\mu$ are the shear and bulk moduli, respectively. Note that the residual on the plastic multiplier, Eq. (41), is written in terms of imposing the yield criterion at the end of each increment, which corresponds to the consistency condition.

An iterative Newton-Raphson procedure is employed to solve the above equation set $[\boldsymbol{R}]^{T}=\mathbf{0}$

$$
[\boldsymbol{V}]_{i+1}=[\boldsymbol{V}]_{i}-\left[\frac{\partial[\boldsymbol{R}]}{\partial[\boldsymbol{V}]_{i}}\right]^{-1}[\boldsymbol{R}]
$$

where the Jacobian matrix $\partial[\boldsymbol{R}] / \partial[\boldsymbol{V}]$ is to be evaluated and the subscript $i$ represents iteration number. To ensure fast convergence a consistent tangent matrix (denoted by $\mathbb{L}^{\tan }$ ) is calculated and used in subsequent global equilibrium steps. Computing $\mathbb{L}^{\text {tan }}$ involves the following steps

$$
\left[\frac{\partial \boldsymbol{V}}{\partial \tilde{\boldsymbol{D}}}\right]=-\left[\frac{\partial[\boldsymbol{R}]}{\partial[\boldsymbol{V}]}\right]^{-1}\left[\frac{\partial \boldsymbol{R}}{\partial \tilde{\boldsymbol{D}}}\right]
$$

where

$$
\left[\frac{\partial \boldsymbol{V}}{\partial \tilde{\boldsymbol{D}}}\right]^{T}=\left[\frac{\partial \tilde{\boldsymbol{\sigma}}^{\prime}}{\partial \tilde{\boldsymbol{D}}}, \frac{\partial \tilde{\sigma}_{\mathrm{m}}}{\partial \tilde{\boldsymbol{D}}}, \frac{\partial f}{\partial \tilde{\boldsymbol{D}}}, \frac{\partial S}{\partial \tilde{\boldsymbol{D}}}, \frac{\partial \bar{\epsilon}}{\partial \tilde{\boldsymbol{D}}}, \frac{\partial \Lambda}{\partial \tilde{\boldsymbol{D}}}\right]
$$

and

$$
\left[\frac{\partial \boldsymbol{R}}{\partial \tilde{\boldsymbol{D}}}\right]^{T}=\left[\frac{\partial \boldsymbol{R}_{\tilde{\boldsymbol{\sigma}}^{\prime}}}{\partial \tilde{\boldsymbol{D}}}, \frac{\partial R_{\tilde{\sigma}_{\mathrm{m}}}}{\partial \tilde{\boldsymbol{D}}}, \frac{\partial R_{f}}{\partial \tilde{\boldsymbol{D}}}, \frac{\partial R_{S}}{\partial \tilde{\boldsymbol{D}}}, \frac{\partial R_{\bar{\epsilon}}}{\partial \tilde{\boldsymbol{D}}}, \frac{\partial R_{\Lambda}}{\partial \tilde{\boldsymbol{D}}}\right]
$$


Note that the Jacobian in Eq. (42) is used again in Eq. (43). Then $\mathbb{L}^{\text {tan }}$ is obtained from (43) as:

$$
\mathbb{L}^{\tan }=\frac{1}{\Delta t}\left(\frac{\partial \tilde{\boldsymbol{\sigma}}^{\prime}}{\partial \tilde{\boldsymbol{D}}}+\boldsymbol{I} \otimes \frac{\partial \tilde{\sigma}_{\mathrm{m}}}{\partial \tilde{\boldsymbol{D}}}\right)
$$

For completeness, the components of the Jacobian and other terms are provided in Appendix B.

On the other hand, the equation for the evolution of void orientation, (32), is integrated using an explicit scheme and an exponential map. Thus, the void axis $\underline{\tilde{n}}_{3}$ is updated following:

$$
\underline{\tilde{n}}_{3}=\operatorname{expm}\left(-\tilde{\Omega}^{\mathrm{p}} \Delta t\right)\left(\underline{\tilde{n}}_{3}\right)_{0}
$$

An implicit update of the void orientation is not used because the corresponding gradient of the exponential map function is quite stiff. Preliminary attempts in this direction have led to overly stiff jacobians, very small time steps and convergence issues. It should be noted that use of explicit updates on internal state parameters is not uncommon $[17,44]$. The algorithm used in the implementation is summarized in Fig. 2.

\section{Illustrations}

Simulation results are shown for two types of calculations. In the first, the constitutive relations are integrated for a material point homogeneously deformed and subjected to various proportional stressing histories. In the second, illustrative boundary-value problems are solved in Abaqus using the developed UMAT both in two and three dimensions. Given the extent of the results, no direct comparisons with voided cell model calculations or experiments are attempted. However, in a subset of calculations, the parameters and loading conditions correspond to situations analyzed in the literature by means of the cell model. This subset is used for benchmarking the model and its implementation. Then, more general situations are considered, which to the authors' knowledge have not yet been considered in cell models, although some situations begin to be simulated in some detail for isotropic matrices [53-55]. Here, considered are situations where the initial orientation of elongated or flat voids is misaligned with either the principal loading axes, the principal directions of orthotropy of the matrix material, or both. Simpler aligned configurations are also considered where the exact void shape evolution is known to depart from spheroidal. The results of these calculations are critically assessed toward employing the constitutive framework, for example in modeling the initial anisotropies that are characteristic of many engineering alloys.

In all calculations, the parameters that remain fixed are: the elastic constants $E=210$ GPa and $\nu=0.3$; the yield stress in some principal direction, say $\underline{e}_{\mathrm{S}}, \sigma_{\mathrm{S}}=420 \mathrm{MPa}$, the hardening exponent $N=0.1$ (unless otherwise noted) and $\epsilon_{0}=0.002$. The parameters that are varied are: the initial void volume fraction, $f_{0}$, the initial void aspect ratio, $w_{0}$, the initial void orientation, $\left(\underline{n}_{3}\right)_{0}$, the anisotropy coefficients of the matrix, $h_{i}$, and where appropriate the stress triaxiality ratio, $\mathcal{T}$. In the finite element calculations of Section 4.8 triaxiality variations are an outcome of the calculations and therefore the effect of some parameters, namely the $h_{i}$ 's, on $\mathcal{T}$ are quantified. In the "material point" calculations, the results are 
presented in terms of an equivalent stress defined as

$$
\sigma_{\mathrm{eqv}}=\sqrt{\frac{3}{2} \boldsymbol{\sigma}^{\prime}: \boldsymbol{\sigma}^{\prime}}
$$

versus an equivalent strain defined as

$$
\varepsilon_{\mathrm{eqv}}=\sqrt{\frac{2}{3} \varepsilon^{\prime}: \varepsilon^{\prime}}, \quad \varepsilon=\int_{0}^{t} \boldsymbol{D} d t
$$

\subsection{Spherical voids in isotropic matrix}

In order to benchmark the code against the Gurson model implementation in the ABAQUS standard library, a version of the code was used where void shape evolution is precluded ( $\dot{S}=\dot{w}=0$ instead of evolution equation (11)). Also, the matrix material is taken to be isotropic and nonhardening $(N=0)$. A biaxial tension simulation was performed in the $x_{1}-x_{2}$ plane so that $\boldsymbol{\sigma}=\sigma \underline{e}_{1} \otimes \underline{e}_{1}+\sigma \underline{e}_{2} \otimes \underline{e}_{2}$ with $\sigma>0$, keeping the stress triaxiality as $\mathcal{T}=2 / 3$.

Fig. 3 shows the comparison between the two implementations in terms of stress-strain curves and porosity evolution. This result shows that when the initial void shape is spherical and constrained to remain so, the model prediction matches that of the Gurson model from the standard library. This and further direct assessments for other conditions provide an effective way for code verification.

\subsection{Spheroidal voids in isotropic matrix: aligned configuration}

Results are here shown for an isotropic von Mises matrix containing spheroidal voids whose common main axis $\underline{n}_{3}$ is aligned with the principal loading direction $\underline{e}_{3}$. The loading is taken to be axisymmetric about $\underline{e}_{3}$. In other words, this is the configuration for which the model rigorously applies since under any other circumstances, the void shape would depart from spheroidal. The effect of void shape is studied by analyzing the cases $w_{0}=1 / 6, w_{0}=1$ and $w_{0}=6$ for various triaxialities, $\mathcal{T}=1 / 3,1$ and 3 , and for two values of the initial porosity $f_{0}=0.0001$ and $f_{0}=0.01$. Similar conditions have been studied in detail by means of cell models [56].

Fig. 4 shows results for a low stress triaxiality, $\mathcal{T}=1 / 3$ (uniaxial loading), up to an equivalent strain of 2.5. It is often reported that void shape effects are important at low triaxialities. This is true but the case $\mathcal{T}=1 / 3$ is quite peculiar. Although large shape changes are induced (Fig. 4e,f), the effect of void shape on the stress-strain curve is negligible (Fig. 4a,b). The small effect seen in the case of a large initial porosity (Fig. 4b) is attributed to a reduction in effective flow strength, roughly by a factor $1-f$. As shown in Figs. 4c,d, the porosity first increases then saturates. This behavior has been discussed before, e.g., $[3,32,34]$. In essence, as the voids elongate their growth rate becomes insensitive to the axial stress; the growth of cylindrical voids is driven, not by mean total stress, but by the mean lateral stress, which is zero in uniaxial loading. The saturation value of $f$ increases with decreasing void aspect ratio, as the void sectional area normal to the loading direction is larger for the oblate void. Also, note that the initial rate of change in the void aspect ratio 
is higher than in steady state: in fact the void initially elongates roughly twice as fast as the "specimen" then elongates roughly at the same rate as the specimen.

Fig. 5 shows the results for a moderate stress triaxiality of $\mathcal{T}=1$. The main difference from the $\mathcal{T}=1 / 3$ case is the porosity-induced softening, notably for $f_{0}=0.01$ (Fig. $5 \mathrm{~b}$ ). In the $f_{0}=0.0001$ case, the softening is not observed until a strain above 1 for $w_{0}=1 / 6$ and even beyond for the spherical or prolate voids (Fig. 5a) despite the two-orders of magnitude increase in porosity (Fig. 5c). The porosity versus strain curves is exponential as would arise from integrating the evolution equation, Eq.(29). They also embody an exponential dependence upon stress triaxiality for $\mathcal{T}$ strictly greater than $1 / 3$, as is well known. Note that the void aspect ratio changes nonlinearly with $\varepsilon_{\text {eqv }}$. The interaction between porosity and the void aspect ratio is highly nonlinear. What is of particulat importance here is that for sufficiently large porosities, oblate voids induce a softening effect that is stronger than a simple $(1-f) \bar{\sigma}$ reduction.

Fig. 6 shows the results for a high triaxiality value, $\mathcal{T}=3$. The overall trend is similar to the $\mathcal{T}=1$ case but here the softening is weakly sensitive to initial void shape. Note that in $(\mathrm{d})$, the oblate $w_{0}=1 / 6$ case does not show the fastest increase anymore; instead it is the prolate $w_{0}=6$ case that has the highest porosity growth, which may appear as counterintuitive. This behavior is explained by the effect of lateral stresses, which are larger in magnitude at high triaxiality, on the growth of prolate voids. Observe the flattening of initially spherical or prolate voids in (e) and (f) despite the fact the major normal stress is along the void axis. The oblate voids, on the other hand, elongate at the beginning then flatten slowly as the plastic strain increases. The results of this section are consistent with previously published results, e.g. $[3,56]$ and demonstrate proper implementation of the model.

\subsection{Spheroidal voids in isotropic matrix: loading perpendicular to the void axis}

Consider yet again some axisymmetric loading about $\underline{e}_{3}$ but now the major stress is perpendicular to the void axis, i.e., $\underline{e}_{3} \perp \underline{n}_{3}$. The axial symmetry of the global problem is now broken but the current void shape is still approximated by the closest spheroidal shape at the same porosity. As in the case of parallel loading, uniaxial loading $(\mathcal{T}=1 / 3)$ is analyzed first. The results are only shown for the $f_{0}=0.0001$ case for brevity, Fig. 7 . The results of parallel loading from Fig. 4 are included for comparison purposes. For the low porosity considered, the load orientation does not affect the stress-strain response, although it significantly affects the evolution of porosity and void shape, as shown in (b) and (c). For the $w_{0}=6$ prolate void, perpendicular loading leads to a greater increase in porosity but saturation occurs after sufficient straining. The void aspect ratio decreases (Fig. 7c), as expected, until it becomes equivalent to a spherical void. At this point, the void axis is determined on the basis of the maximum stretch, which here is along $\underline{e}_{3}$, as explained at the end of Section 3.1. From then on, the void is approximated as a prolate void with an axis $\underline{n}_{3}=\underline{e}_{3}$. Under the type of loading considered, the prolate void elongates. This explains the apparent slope discontinuity in Fig. 7c. For the $w_{0}=1 / 6$ oblate void, perpendicular loading leads to a steady increase in porosity, in contrast with the case of parallel loading. Also, the void 
develops an effectively more oblate shape as $S$ decreases, consistent with physical intuition. The results in Fig. 7b are qualitatively rationalized in terms of effective void cross-sectional area normal to the main loading.

Next, consider the case of higher triaxialities. Only the case of $\mathcal{T}=1$ is shown for brevity. Comparison between the parallel and perpendicular loadings is shown in Fig. 8. Observe the marked difference in equivalent stress among the various cases. In increasing order, the rate of growth of porosity follows the pattern $\left(w_{0}=1 / 6\right.$, perpendicular $),\left(w_{0}=1 / 6\right.$, parallel $)$, $\left(w_{0}=6\right.$, perpendicular $),\left(w_{0}=1\right)$ and $\left(w_{0}=6\right.$, parallel $)$, which is almost the same as the order of the void sectional area normal to the major load direction, the exception being the first two. In fact, the initial rates of $f$ follow the above order. Although not clearly seen in Fig. $8 \mathrm{~b}$, the porosity curves of the $\left(w_{0}=1 / 6\right.$, perpendicular) and $\left(w_{0}=1 / 6\right.$, parallel) cases cross each other at $\varepsilon_{\text {eqv }} \approx 0.25$. This trend for $w_{0}=1 / 6$ can be rationalized on the basis of void shape evolution, Fig. 8c. In the parallel loading case, the void opens up and eventually becomes prolate. By way of contrast, in the perpendicular loading case, the initially oblate void remains oblate (in the sense of an effective shape). The observed trend is then explained by the growth rate of flat voids being faster than that of prolate voids of comparable volume. For the initially prolate void $\left(w_{0}=1 / 6\right)$ loaded perpendicular to its axis, the reversal in void shape evolution after passing through $w=1$ (sphere) is due to a change in the void axis (see $\mathcal{T}=1 / 3$ case above).

\subsection{Spheroidal voids in isotropic matrix: misaligned loadings}

Consider now loadings that are neither parallel nor perpendicular to the common axis of voids. Let $\theta$ be the current angle ${ }^{3}$ between the loading axis $\underline{e}_{3}$ and the void axis $\underline{n}_{3}$ with $\theta_{0}$ denoting its initial value.

Fig. 9 shows results obtained for an initially prolate void with a triaxiality $\mathcal{T}=1$. Three initial void orientations are considered, $\pi / 8, \pi / 4$ and $3 \pi / 8$. In addition to the stress-strain curves, porosity evolution and void shape change, the evolution of void orientation $\theta$ is also shown in part $(d)$. Altough not included in the figure, the results previously shown for the parallel and perpendicular loading cases encompass the three orientations shown in terms of rate of growth of porosity. The evolution of void shape for the slightly misaligned void $\left(\theta_{0}=\pi / 8\right)$ is closest to the parallel loading case of Fig. 8 , which corresponds to $\theta_{0}=0$ but the rate of elongation is slower. On the other hand, the evolution of void shape for the strongly misaligned void $\left(\theta_{0}=3 \pi / 8\right)$ is closest to the perpendicular loading case of Fig. 8, which corresponds to $\theta_{0}=\pi / 2$, but here a non-monotone evolution of void shape is recorded without the void going through a spherical shape or abrubtly changing its void axis. The latter behavior is explained by examining the changes in void orientation, Fig. 9d. Misaligned prolate voids tend to rotate so as to align their axis with the major normal stress $(\theta \rightarrow 0)$. This is seen in the asymptotic behavior in Fig. 9d, irrespective of the initial orientation. In the early stages of deformation, when the void is sufficiently inclined $\left(\theta_{0}=3 \pi / 8\right)$, its shape evolves toward spherical as the void opens up. This explains the initial decrease in void aspect ratio, Fig. 9c. However, when the void has aligned itself with the major stress it

\footnotetext{
${ }^{3}$ For a fixed global axis $\underline{e}_{3}$, the void axis may lie on any generatrice of the cone defined by solid angle $\theta$. For an isotropic matrix the specific choice of $\underline{n}_{3}$ does not matter.
} 
elongates, which is the expected trend for a prolate void deformed at a triaxiality of $\mathcal{T}=1$. This explains the non-monotone evolution of $S$ in Fig. 9c for the strongly misaligned void. More generally, the evolution of void shape for misaligned prolate voids results from the competition between the tendency to become spherical due to the effective void-sectional area beging larger and the tendency to elongate subsequent to alignment with the major stress. Note that the reversal from void flattening to elongation in Fig. 9c begins before the void has completely oriented itself along the major stress. Typically, the reversal seems to occur when the voids are about $\sim 25-35^{\circ}$ from the loading axis.

Results for an initially misoriented oblate void are gathered in Fig.10, for the same triaxiality $\mathcal{T}=1$. As shown in Fig.10d, the angle $\theta$ evolves toward $90^{\circ}$, irrespective of initial orientation $0<\theta_{0}<90^{\circ}$. Unlike prolate voids, oblate ones rotate to align their $\underline{n}_{3}$ axis perpendicular to the major stress. What is common to both cases is that misoriented voids align themselves to minimize the sectional area normal to the major loading direction. The evolution of the void aspect ratio (Fig.10c) is rationalized on the basis of previous results for parallel and perpendicular loading and the evolution of void orientation. The shape parameter $S$ gets closer to zero at the beginning of the simulations, i.e., the void tend to becoming spheres due to the triaxial loading that expands the void in all directions. Once the void passes a certain orientation, which seems to be within 30 degrees away from the asymptotic direction, $S$ evolves in the opposite direction, i.e., gets away from zero (sphere).

The larger the initial misalignement, the faster the porosity growth (Fig. 9b and 10b). The effect of misalignment is smaller in the $w_{0}=1 / 6$ case. However, when the extreme cases of parallel and perpendicular loading are included the effects are comparable over the full range of orientations. This illustrates once again that the interactions between porosity growth, void shape changes and void reorientations are strongly nonlinear.

\subsection{Spherical void in anisotropic matrix}

The effect of plastic anisotropy of the matrix is first investigated for the case of spherical voids embedded therein. Focus is on the case $\mathcal{T}=1$ and $f_{0}=0.001$. One of the principal directions of orthotropy, here $\underline{e}_{S}$, is taken along the main loading axis $\underline{e}_{3}$. Simulations were carried out for three anisotropic matrices, see Table 1. Previous cell model calculations [35] assuming similar initial parameters were performed for the transversely isotropic matrices (MAT1 and MAT2 in the table, the notation being taken from [39]). Here, in addition, a 3D anisotropic case is considered as MAT3. In this case, the idealization of the void shape as spheroidal is an approximation: even under axially symmetric loading the void would develop into an ellipsoid (more generally a 3D void) because of matrix anisotropy. The stress versus strain curves and corresponding porosity evolution curves are shown in Fig. 11. The trends obtained for materials MAT1 and MAT2 are similar to the figure 13 in [39], which enables further verification of the proposed implementation. The new results for MAT3 are intermediate between the reference isotropic matrix and MAT1.

The interpretation of the observed strong effect of matrix anisotropy on void growth follows the rationale developed by Benzerga and Besson [35] and further expanded in [57]. It is emphasized here for completeness along with other details. First, note that a large value of a Hill coefficient in a given direction means that the material is weak/soft in that direction. The isotropic matrix, MAT1 and MAT2 have the same Hill parameters $h_{\mathrm{L}}, h_{\mathrm{T}}$ and $h_{\mathrm{S}}$ in 
the three principal directions, L, T, and $\mathrm{S}$ (Table 1). The triaxial loading in the simulations does not involve any shear stress, i.e., $\sigma_{12}=\sigma_{13}=\sigma_{23}=0$. Yet, the responses of the three materials are different, Fig. 11. The reason for this is that plastic flow in the absence of voids, i.e., $f=0$, exhibits the same response for the isotropic, MAT1 and MAT2 matrices in case of shear-free loadings. However, with the voids present the microscopic stress state within the RVE (Representative Volume Element which contains a void and the matrix) involves non-zero shear stress components even when the macroscopic stress state does not involve any. The effect of the inhomogeneous stress state including shear stress components within the RVE is taken into account by the second term in the yield criterion Eq.(5), which writes:

$$
2(g+1)(g+f) \cosh \left(\kappa \frac{\boldsymbol{\sigma}: \boldsymbol{X}}{\bar{\sigma}}\right)
$$

The interaction between plastic anisotropy and porosity is taken into account through the parameter $\kappa$, whose full expression is given in Appendix A. In the case of a spherical void, $\kappa$ reduces to the following simplified form first derived in [35]:

$$
\kappa^{\mathrm{BB}}=\frac{3}{2}\left[\frac{2}{5} \frac{h_{\mathrm{L}}+h_{\mathrm{T}}+h_{\mathrm{S}}}{h_{\mathrm{L}} h_{\mathrm{T}}+h_{\mathrm{T}} h_{\mathrm{S}}+h_{\mathrm{S}} h_{\mathrm{L}}}+\frac{1}{5}\left(\frac{1}{h_{\mathrm{TS}}}+\frac{1}{h_{\mathrm{SL}}}+\frac{1}{h_{\mathrm{LT}}}\right)\right]^{-1 / 2}
$$

The above equation indicates that $\kappa$ is nonlinearly related to the $h_{i}$ 's. As shown in Eq. (29), the rate of porosity evolution increases exponentially with $\kappa$. Therefore, large values of $h_{\mathrm{LT}}, h_{\mathrm{TS}}$ and $h_{\mathrm{SL}}$ can induce large porosity-induced softening, a behavior that has been checked using cell model calculations [35,57]. Interestingly, although Eq.(51) is nonlinear in the Hill coefficients, the overall trend can be explained by simply summing up all the Hill coefficients. With this rationale, MAT1 is the softest (weakest) material and MAT2 is the hardest (strongest) material despite the same normal Hill coefficients $\left(h_{\mathrm{L}}, h_{\mathrm{T}}\right.$ and $\left.h_{\mathrm{S}}\right)$; The order of the sum of the Hill coefficients is as follows, 8.666 (MAT1), 7.269 (MAT3), 6 (Isotropic) and 5 (MAT2). The order of porosity is the same as the order of the sum of the Hill coefficients and the order of strength is opposite to the order of the sum of the Hill coefficients.

\subsection{Spheroidal voids in anisotropic matrix: parallel loading}

With the above results in mind for the net effect of plastic anisotropy on void growth, the combined effects of the two sources of macroscopic anisotropy (void shape and matrix anisotropy) are now considered. As in the previous section the loading is parallel to principal direction $\mathrm{S}\left(\underline{e}_{3}=\underline{e}_{\mathrm{S}}\right)$. In addition, the major applied load is parallel to the void axis $\left(\underline{e}_{3}=\underline{n}_{3}\right)$, as in Section 4.2 , Here again, $\mathcal{T}=1$ and $f_{0}=0.001$.

Fig.12 shows the results corresponding to MAT1, MAT2 and the isotropic matrix. The overall trend is similar to the figure 13 in [39], which provides further verification of the implementation regarding the anisotropy part. In MAT2, the strongest matrix, the effect of the initial void shape is small as indicated by the small difference in porosity evolution. On the other hand, in MAT1, the weakest matrix, the effect of the initial void shape is significant. Porosity evolution is faster in the oblate case $\left(w_{0}=1 / 2\right)$ than the prolate case

$\left(w_{0}=2\right)$, as in the isotropic matrix case, Fig.8. Observe the faster increase of $w$ in MAT2 
than MAT1, Fig.12c. This means that void becomes prolate faster in the stronger matrix (MAT2) than the weaker matrix (MAT1). The stronger matrix squeezes void faster by its stronger shear strength $\left(h_{\mathrm{TS}}\right.$ and $\left.h_{\mathrm{SL}}\right)$. This leads to a nontrivial coupling between void shape and plastic anisotropy effects.

Fig.13 shows the results for MAT3. MAT3 is weaker than the isotropic matrix, consistent with the results obtained for spherical voids, Fig.11. The sum of the Hill coefficients of MAT3 (7.269) is larger than that of the isotropic matrix (6), which evolves porosity faster and thus softens the material faster in MAT3. As shown in Fig.4 to Fig.8, porosity evolution is faster in the oblate case $\left(w_{0}=1 / 2\right)$ than the prolate case $\left(w_{0}=2\right)$. Note that the void shape becomes prolate faster in the isotropic matrix than MAT3; the stronger matrix (the isotropic matrix) squeezes void faster than the weaker matrix (MAT3) by its stronger shear strength $\left(h_{\mathrm{TS}}\right.$ and $\left.h_{\mathrm{SL}}\right)$.

\subsection{Spheroidal voids in anisotropic matrix: misaligned loadings}

Here, the effect of matrix anisotropy on the evolution of void shape and orientation under misaligned loading conditions is investigated using $\mathcal{T}=1, f_{0}=0.0001$ and $\underline{e}_{3}=\underline{e}_{\mathrm{S}}$. However, the void axis makes an angle $\theta_{0}$ with the major applied load, as in Section 4.4. The strongest matrix (MAT2) and the weakest (MAT1) and two different initial void aspect ratios, $w_{0}=6$ and $w_{0}=1 / 6$ are employed for comparison. Three different initial misaligned angles of $\theta_{0}=\pi / 8, \theta_{0}=\pi / 4$, and $\theta_{0}=3 \pi / 8$ are simulated.

The results are shown in Figs.14-17. Regardless of material type and initial orientation $\left(\theta_{0} \neq \pi / 2\right)$, the final orientation of the void axis $\underline{n}_{3}$ rotates to the main loading axis in the case of the $w_{0}=6$ prolate voids and to a direction perpendicular to the main loading axis in the case of the oblate $w_{0}=1 / 6$. This trend is consistent with that obtained for the isotropic matrix, Figs.9 and 10. However, the extent of the effect of initial misalignment on damage growth and the stress-strain response depends on both the matrix and initial void shape. Indeed, the influence of $\theta_{0}$ is strong for the MAT1 matrix with prolate voids (Fig.14), small for the same matrix with oblate voids (Fig.15), negligible for the MAT2 matrix with prolate voids (Fig.16), and small again for the same matrix with oblate voids (Fig.17). These results illustrate nontrivial coupling between matrix anisotropy, void shape and void orientation effects.

\subsection{Finite element simulations}

A series of calculations were carried out using tensile specimens under axisymmetric, plane strain and fully 3D conditions. The parameter space investigated is not as wide as for single element computations. The aim here is to demonstrate boundary-value problem solutions using the proposed constitutive framework. The specimen geometry is shown in Fig.18 and corresponds to an ASTM standard with relative dimensions $L_{0}=57, W_{0}=19, t_{0}=3.2$, $b w=29$ and $R=76, t_{0}$ denoting the thickness where relevant. To reduce the computational cost, only a small portion of the grip section is modeled such that $l_{0}=136$.

For the 3D simulations, a quarter of the specimen is used as shown in Fig. 19a utilizing 
the symmetry with respect to the planes $x_{1}=0$ and $x_{3}=0$. The boundary conditions are:

$$
\begin{gathered}
T_{1}\left(x_{1}, \frac{l_{0}}{2}, x_{3}\right)=0, \quad \dot{u}_{2}\left(x_{1}, \frac{l_{0}}{2}, x_{3}\right)=\dot{U}, \quad T_{3}\left(x_{1}, \frac{l_{0}}{2}, x_{3}\right)=0 \\
T_{1}\left(x_{1},-\frac{l_{0}}{2}, x_{3}\right)=0, \quad \dot{u}_{2}\left(x_{1},-\frac{l_{0}}{2}, x_{3}\right)=0, \quad T_{3}\left(x_{1},-\frac{l_{0}}{2}, x_{3}\right)=0 . \\
\dot{u}_{1}\left(0, x_{2}, x_{3}\right)=0, \quad T_{2}\left(0, x_{2}, x_{3}\right)=0, \quad T_{3}\left(0, x_{2}, x_{3}\right)=0 \\
T_{1}\left(x_{1}, x_{2}, 0\right)=0, \quad T_{2}\left(x_{1}, x_{2}, 0\right)=0, \quad \dot{u}_{3}\left(x_{1}, x_{2}, 0\right)=0 .
\end{gathered}
$$

where $T_{i}$ denote the traction components and $u_{i}$ the displacement components. Also, $\dot{U}$ is a prescribed displacement rate. A uniform grid is used in the gauge section, Fig. 19a, using 14160 eight-noded brick elements (C3D8).

For the plane strain and axisymmetric tensile simulations, the in-plane dimensions are the same as those of the 3D standard specimen, Fig. 18. Only one half of the plane strain specimen is analyzed utilizing the symmetry with respect to the $x_{1}=0$ plane. For both cases, the main boundary conditions are as follows

$$
\begin{gathered}
T_{1}\left(x_{1}, \frac{l_{0}}{2}\right)=0, \quad \dot{u}_{2}\left(x_{1}, \frac{l_{0}}{2}\right)=\dot{U} \\
T_{1}\left(x_{1},-\frac{l_{0}}{2}\right)=0, \quad \dot{u}_{2}\left(x_{1},-\frac{l_{0}}{2}\right)=0 . \\
\dot{u}_{1}\left(0, x_{2}\right)=0, \quad T_{2}\left(0, x_{2}\right)=0 .
\end{gathered}
$$

Fig. 19b shows the mesh employed in the 2D simulations. A total of 4720 four-noded CAX4 and CPE4 elements are used for the axisymmetric and plane strain simulations, respectively.

In all simulations, the initial porosity value employed is $f_{0}=0.001$ and the initial void aspect ratio is set to $w_{0}=1$. The elastic and flow parameters are those specified at the beginning of Section 4. Two matrix materials are used: the isotropic material, for reference, and MAT3; see Table 1. For the anisotropic material MAT3, the principal directions of orthotropy, $\mathrm{L}, \mathrm{T}$ and $\mathrm{S}$, are set to align with the $x_{1}, x_{2}$ and $x_{3}$ directions, respectively. Clearly, an axisymmetric calculation for MAT3 would be irrelevant. However, a plane strain calculation is carried out with the $x_{1}-x_{2}$ plane of deformation being identified with the $\mathrm{L}-\mathrm{T}$ plane.

Fig. 20 shows the global responses in terms of normalized load versus nominal strain measure $E^{\text {nom }}=U_{g} / L_{0}$, where $U_{g}$ is the displacement of the gauge section having length $L_{0}$ (see Fig. 18). For the isotropic material, the maximum load is higher in plane strain than under axisymmetric tension by roughly a factor of 1.15 at initial yield and 1.173 subsequently, up to necking (Fig. 20a). This is due to the triaxial state of stress in plane strain. The initial load drop is due to necking but subsequent softening occurs faster in plane strain. This is a signature of the higher triaxiality effect on damage growth, which will be analyzed further below. As a result, the responses for the plane strain and axisymmetric specimens intersect after sufficient damage-induced softening.

The plane strain response of the anisotropic material MAT3 is softer than that of the reference isotropic material, Fig. 20a. At first sight, this is surprising since MAT3 is stronger along the $\mathrm{T}$ direction $\left(h_{T}=0.778\right)$, which is the loading direction, $x_{2}$. The trend is actually 
reversed in the 3D thin specimen, as shown in Fig. 20b. In general, however, the yield strength of an anisotropic material loaded along a principal direction depends on all three principal Hill coefficients, not only $h_{T}=0.778$. The weight of each coefficient depends on the stress state. For instance, in axisymmetric tension it is the combination $4 h_{T}+h_{L}+h_{S}$ that matters, whereas in plane stress the $h_{S}$ coefficient does not affect yielding.

To gain insight into the differences observed among all specimens, spatial distributions of key internal variables are now examined. Fig. 21 shows contours of porosity $f$ and void aspect ratio $S=\ln w$ for the plane strain tensile specimens at $E \equiv \ln \left(A_{0} / A\right)=1.0$. Here, $A$ and $A_{0}$ respectively denote the current and initial areas at the neck. The development of porosity damage occurs much faster in the isotropic material than in material MAT3 (compare (a) and (c)). This is opposite to what was observed in Fig. 11. This hints at a nontrivial coupling between plastic constraint and plastic anisotropy effects on ductile damage evolution. This issue will be analyzed more quantitatively below. From the contours of void aspect ratio, (b) and (d), it is clear that voids elongate more at the center of the specimen when the anisotropic material MAT3 is used. Note that node-interpolated values are shown for the porosity while element-averaged values are shown for the void aspect ratio to display the results in various ways.

Fig. 22 shows contours of porosity and void aspect ratio for the axisymmetric tensile specimen at the same value of logarithmic strain as in Fig. 21, i.e. $E=1$. Recall that only the isotropic matrix is employed in the axisymmetric simulations. At the same overall strain level, the porosity levels are lower in the axisymmetric specimen compared with the plane strain specimen, which is expected based on triaxiality levels. For the same reason, void elongation is more significant in the axisymmetric specimen.

Fig. 23 shows contours of porosity for the 3D tensile specimen simulation, again at $E=1$ as above, but also at $E^{\text {nom }}=0.15$. The contours of the void aspect ratio are not shown for brevity since the patterns are similar to those of the axisymmetric case. Unlike in the plane strain specimens, porosity evolution is faster in MAT3, a trend which is consistent with the material point simulations in Fig. 11. This trend is already evident at incipient necking $\left(E^{\text {nom }}=0.15\right)$ and further develops at large strains $(E=1)$. For both materials, necking occurs almost at the same time in-plane and out-of-plane, with the latter being more intense.

In order to quantify further the above trends, Fig. 24 shows the evolution of porosity $f$, stress triaxiality $\mathcal{T}$, and void aspect ratio $S=\ln w$ at the center of each specimen with the logarithmic strain $E$. First, consider the case of the isotropic material. Prior to necking, there is little porosity growth (Fig. 24a), in fact none in the axisymmetric and 3D specimens, although the voids elongate in all cases (Fig. 24c). In plane strain, stress triaxiality is about 0.4875 , from the elastic analytic expression $\mathcal{T}=(1+\nu) /\left(3 \sqrt{\nu^{2}-\nu+1}\right)$ for $\nu=0.3$. In 3D and axisymmetry, stress triaxiality starts at $1 / 3$, which is the value for homogeneous uniaxial tension. Post-necking, the higher triaxiality prevailing in the plane strain specimen (Fig. 24b) causes a much greater porosity growth. The 3D and axisymmetric curves are close to each other. Upon continued straining, stress triaxiality becomes higher in the axisymmetric specimen than in $3 \mathrm{D}$, the out-of-plane stress, $\sigma_{33}$, being smaller in $3 \mathrm{D}$ specimen due to the small thickness.

With the reference isotropic material in mind, consider next the simulations involving material MAT3. The expectation based on homogeneous axisymmetric deformation results (Fig. 11) is that MAT3 be less resistant to void growth than the isotropic material. This is 
exactly what is observed in the 3D specimens. However, in plane strain the trend is reversed and the rate of porosity growth is much slower in MAT3. This behavior is explained in part by the lower triaxiality prevailing in the MAT3 plane strain specimen (Fig. 24b). Unlike in the material point simulations of Fig. 11 where the triaxiality was held constant, here spatial and temporal variations of $\mathcal{T}$ are the outcome of the boundary-value problem solution as affected by plastic flow anisotropy. Thus, plastic anisotropy effects on void growth manifest in quite different ways depending on the level (and direction) of plastic constraint.

Prior to necking, the evolution of void aspect ratio (at the center) is the same for all specimens, Fig. 24c. Referring to evolution equation (11), it is noted that the contribution of the term proportional to the mean plastic dilatation $D_{\mathrm{m}}^{\mathrm{p}}$ is either nil (zero porosity growth in the 3D and axisymmetric specimens) or negligibly small (nonzero but small porosity growth in the plane strain bar due to $\mathcal{T}>1 / 3$ ). Also, the contribution of the triaxiality-dependent heuristic function $k_{\mathcal{T}}$ in the deviatoric term is small for $\mathcal{T}$ below, say 0.5 . Since prior to necking the logarithmic strain $E$ is a measure of true strain, it is expected that the rate of void shape change be the same in all uniaxial specimens, irrespective of constraint. Beyond the onset of necking, two factors contribute to the differences seen among all specimens. As the triaxiality rises above 0.5 in all specimens, the contribution of heuristic function $k_{\mathcal{T}}$ increases. Most importantly, the increase in porosity induced by the rising triaxiality affects void shape in two ways: both through the $D_{\mathrm{m}}^{\mathrm{p}}$ and heuristic function $k_{\mathrm{f}}$ in (11). In plane strain, the void aspect ratio is the smallest because of the higher triaxiality prevailing therein. The effect of plastic anisotropy on the evolution of $w$ is (for the loading paths considered and probably most others) either small in the 3D thin specimen or moderate in the plane strain specimen. Such trends should be compared more carefully with unit cell calculations.

\section{Discussion}

Constitutive relations have been presented and implemented to model damage accumulation in ductile materials. They extend the well known Gurson model to a wider space of damagerelated microstructural parameters. Models with the capability of representing void shape changes and reorientations had been developed in [32,34,58,59], [8] and [10,46,49] and applied in e.g. $[13,42-44,60]$. The model used here captures these as well as matrix anisotropy effects, which strongly couple with void shape effects. The plastic anisotropy parameters may be viewed as plasticity-related microstructural parameters, although here they were taken as non-evolving. Because the model is derived from homogenization theory, the mathematical expressions of the microstructure-dependent functions entering the model are quite involved (see Appendix). However, the model involves a priori no adjustable parameter and, since all functions are analytic, there is no difficulty in implementing the model.

The anisotropy considered in this paper is limited to the quadratic Hill model. There are other important models that deal with plastic anisotropy in different ways. It is straightforward to replace the quadratic term of the new potential with other forms representing approximately the plastic anisotropy of the fully dense matrix, as in previous implementations in an object-oriented code [42]. One difficulty, however, would be to obtain from first principles the functional dependence of the criterion parameters, such as $\kappa$, upon the anisotropy parameters. In addition, the anisotropy axes of the matrix material were taken 
to rotate with the material for simplicity. In many instances, not only the axes can rotate independent of the material, as a result of say grain rotations in polycrystals, the magnitudes of the anisotropy coefficients evolve due for example to an evolving slip system acitivity [61]. Crystal plasticity based damage models naturally take into account the evolution of (matrix) anisotropy, e.g., [37]. However, their use in large-scale simulations of ductile fracture remains to be demonstrated. Alternatively, coupled plasticity and damage formulations accounting for the plastic spin have recently been proposed [62].

While the model has been verified to some extent against cell model calculations, e.g., [57,63], a quantitative assessment of the model predictive abilities over the full range of internal paramaters is a daunting task, especially for misaligned orientations. Therefore, emphasis was laid here on trends obtained for loading cases and parameter values heretofore not considered in the literature. Note that in practice, the model is expected to be employed in situations where the underlying assumptions, e.g. a spheroidal void shape, are known to break down ${ }^{4}$. Under such circumstances, it was important to critically assess the predictions made using the model in the absence of exact solutions. Along these lines, it is important to note that the results shown in Fig. 5 for void shape effects in the absence of plastic anisotropy can be further improved by introducing a void-shape dependent Tvergaard parameter, $q_{w}$, as suggested in [64]. Indeed, the results in Fig. 5 suggest a much smaller difference between the $w_{0}=1$ and $w_{0}=6$ cases than inferred from cell model calculations [56]. Heuristic parameter $q_{w}$ is key to obtaining improved results. It has not been used here because there is a priori no basis for assessing its potential dependence upon the plastic anisotropy coefficients or loading orientation. Following [42] it can be considered as independent of these parameters; hence using:

$$
q_{w}=1+(q-1) / \cosh S
$$

where $q$ is the value taken by $q_{w}$ for a spherical void.

The model can be used to describe damage processes involving significant void rotations (e.g., Fig. 14), as would prevail under shear-dominated loadings, or represent anisotropic damage evolution depending on the main loading direction (e.g., Fig. 8). The latter capability was used by Benzerga et al. [43] to model the anisotropic fracture of steel for situations where the principal loading directions were aligned with the principal directions of the microstructure. The formulation presented here proves promising to extend the modeling range to off-axes loadings where shear loads are induced and void rotations may play a prominent role. This aspect of the model was illustrated here for homogeneously deformed material points assuming proportional stressing histories. It should be emphasized that in the finite element simulations of Section 4.8, the necking induced surface curvatures lead to void rotations near the surfaces, consistent with experimental observations [65]. However, these are not as important as the void rotations expected under off-axes loadings. It remains to be seen how the model can predict damage accumulation under such circumstances.

Finally, the constitutive relations presented focus on plasticity and damage by void distortion (growth/shrinkage and rotation) but do not deal with the initial and final stages of damage. The effect of progressive cavitation through void nucleation rules may be incorporated with no difficulty, by amending equation (10) with a nucleation term, for example

\footnotetext{
${ }^{4}$ The use of the Gurson model is no different in this regard. One can hardly think of any practical loading where spherical voids would retain their initial shape.
} 
following [66]. Most importantly, the model should be supplemented with a void coalescence model using either the phenomenological approach in [2] or micromechanical models that include evolution equations for the internal variables, as initiated in [67] and recently further developed on more rigorous grounds $[68,69]$ including for combined tension and shear loading [70]. Here, without such models, the loss of stress carrying capacity follows directly from damage induced softening. In the full boundary-value problem solutions of Section 4.8, the large strains attained at maximum-damage locations lead to significant element distortion. The use of void coalescence models would enable a faster drop in load bearing capacity, in keeping with physical experiments, hence alleviating some of the numerical difficulties associated with modeling damage to fracture initiation transitions.

\section{Conclusion}

A computational procedure was developed to implement a recent anisotropic model of porous material plasticity that takes into account the concurrent effects of void shape and matrix orthotropy [39] in a finite deformation setting. The procedure is based upon the corotational formulation of the constitutive relations. A semi-implicit scheme was employed to integrate the latter (explicit only in the void rotation) and the Newton-Raphson method was used to obtain the converged state variables and the consistent tangent matrix needed to solve the global force-displacement matrix equation in nonlinear boundary value problems. Using the proposed procedure, implementation of the model enabled a series of simulations for various combinations of stress triaxiality, void aspect ratio, porosity, loading directions and Hill anisotropy parameters to be performed. In addition, using a concurrent finite-element implementation a series of preliminary calculations were carried out to investigate the effect of stress and strain state in uniaxial bars on damage accumulation up to large strains. The main findings are summarized as follows.

- Code verification was addressed by comparing computation results with those obtained using the Gurson model of the standard library of Abaqus as well as available results from the literature. Then, model predictions for various loading orientations and plastic anisotropies were assessed.

- Under uniaxial axisymmetric loading, voids elongate indefinitely with no incidence on the stress-strain curve unless (i) the initial porosity is sufficiently large; (ii) the initial void shape is suffiently oblate; or (iii) the loading direction is normal to the void axis for oblate voids. In practice, uniaxial bars of ductile materials are rarely observed to fail prior to necking. The results obtained for perpendicular loading rationalize the possibility of fracture under pure tension, for example if void nucleation occurs by particle cracking and the cracks are closely spaced.

- For moderate to high triaxialities, damage accumulation occurs at a faster rate for perpendicular loading (i.e. when the major normal stress is perpendicular to the void axis) irrespective of void aspect ratio.

- Under off-axes loadings, prolate voids rotate so as to align with the major load direction whereas oblate voids rotate to align perpendicular to the major load. The rate of 
realignment depends on the initial orientation and stress triaxiality, and to a lesser extent, on plastic anisotropy and porosity.

- Plastic anisotropy has a large effect on the net rate of void growth, irrespective of load orientation and stress triaxiality. This result is a major finding due to homogenization theory and scale transition. In particular, it could not be arrived at by phenomenolgocal models of continuum thermomechanics as discussed in [35].

- Concurrent effects of plastic constraint and anisotropy lead to non trivial couplings. While the scalar invariant (51) of the anisotropy tensor provides a simple means of quantifying the effect of anisotropy on porosity growth rates, in general the effect of plastic anisotropy also manifests through (i) different evolution of stress triaxiality, which ultimately strengthens or counteracts the effect of $\kappa^{\mathrm{BB}}$; and (ii) the interaction between plastic constraint and anisotropy, as illustrated by the finite element simulations.

- In order to be used for ductile fracture simulations, the model should be supplemented with a void coalescence model. This would in turn limit the strains at which complete loss of load carrying capacity occurs at the level of an integration point.

- As it stands, the formulation lacks a length scale. In the absence of strong macroscopic gradients of mechanical fields, as in the simulations presented here, the results are not so much mesh-sensitive. More generally, enhanced formulations of the constitutive relations are needed to address the ill-posedness of the incremental problem when softening occurs.

\section{Acknowledgments}

SK acknowledges support from the STEP (Seed grants for Transitional and Exploratory Projects) grant at Southern Illinois University Edwardsville. AAB acknowledges support from the Lawrence Livermore National Security, LLC under Master Task Agreements No. B599687 and B602391 and from the National Science Foundation under grant CMMI-1563580. 


\section{Appendix A. Criterion Parameters}

The parameters that appear in criterion (5) are functions of porosity $f$, void aspect ratio $w$ and the Hill anisotropy coefficients. They are $g, \kappa, \alpha_{2}, \eta, C$, and $\alpha_{1}$. The "secondary porosity" $g$ is non-zero for oblate voids only:

$$
g=0 \quad(\mathrm{p}) ; \quad g=\frac{e_{2}^{3}}{\sqrt{1-e_{2}^{2}}}=f \frac{e_{1}^{3}}{\sqrt{1-e_{1}^{2}}}=f \frac{\left(1-w^{2}\right)^{\frac{3}{2}}}{w}
$$

where (p) and (o) stand for "prolate" and "oblate", respectively. Also, $e_{1}$ and $e_{2}$ are respectively the eccentricities of the void and the outer boundary of the spheroidal RVE used in deriving the model. Both are implicit functions of $f$ and $w$ :

$$
e_{1}^{2}=\left\{\begin{array}{ll}
1-\frac{1}{w_{2}^{2}} & (\mathrm{p}) \\
1-w^{2} & (\mathrm{o})
\end{array}, \quad \frac{\left(1-e_{2}^{2}\right)^{n}}{e_{2}^{3}}=\frac{1}{f} \frac{\left(1-e_{1}^{2}\right)^{n}}{e_{1}^{3}}, \quad n= \begin{cases}1 & (\mathrm{p}) \\
1 / 2 & (\mathrm{o})\end{cases}\right.
$$

Also,

$$
\kappa=\left\{\begin{array}{l}
\frac{3}{h}\left\{1+\frac{h_{t}}{h^{2} \ln f} \ln \frac{1-e_{2}^{2}}{1-e_{1}^{2}}\right\}^{-1 / 2} \\
\frac{3}{h}\left\{1+\frac{\left(g_{f}-g_{1}\right)+\frac{4}{5}\left(g_{f}^{5 / 2}-g_{1}^{5 / 2}\right)-\frac{3}{5}\left(g_{f}^{5}-g_{1}^{5}\right)}{\ln \frac{g_{f}}{g_{1}}}\right\}^{-1}
\end{array}\right.
$$

where

$$
g_{f} \equiv \frac{g}{g+f}, \quad g_{1} \equiv \frac{g}{g+1}
$$

and the anisotropy factors $h$ and $h_{t}$ are defined by

$$
\begin{gathered}
h=2\left[\frac{2\left(\hat{h}_{11}+\hat{h}_{22}+\hat{h}_{33}-\hat{h}_{23}-\hat{h}_{31}-\hat{h}_{12}\right)+3\left(\hat{h}_{44}+\hat{h}_{55}+\hat{h}_{66}\right)}{15}\right]^{1 / 2} \\
h_{t} \equiv h^{2}-\frac{3}{4}\left(\hat{h}_{11}+\hat{h}_{22}+2 \hat{h}_{66}-2 \hat{h}_{12}\right)
\end{gathered}
$$

where $\hat{h}_{i j}$ denote the components of the fourth order tensor $\hat{\mathrm{h}}$, formal inverse of $\mathbb{h}[35]$

$$
\hat{\mathbb{P}} \equiv \mathbb{J}: \hat{\mathbb{h}}: \mathbb{J}, \quad \mathbb{p}: \hat{\mathbb{p}}=\hat{\mathbb{P}}: \mathbb{p}=\mathbb{J}
$$

expressed in Voigt notation, in the frame $\left(\underline{n}_{1}, \underline{n}_{2}, \underline{n}_{3}\right)$ of Fig. 1 . Tensors $\mathbb{h}$ and $\hat{\mathrm{h}}$ are symmetric positive definite, i.e. $h_{i j k l}=h_{j i k l}=h_{i j l k}=h_{k l i j}$ and $\forall \boldsymbol{\sigma} \neq \mathbf{0}, \boldsymbol{\sigma}: \mathbb{h}: \boldsymbol{\sigma}>0$. In the frame $\left(\underline{e}_{\mathrm{L}}, \underline{e}_{\mathrm{T}}, \underline{e}_{\mathrm{S}}\right)$ the six Hill coefficients are denoted as $h_{\mathrm{L}}, h_{\mathrm{T}}, h_{\mathrm{S}}, h_{\mathrm{TS}}, h_{\mathrm{SL}}$ and $h_{\mathrm{LT}}$. Next,

$$
\alpha_{2}=\left\{\begin{array}{c}
\frac{\left(1+e_{2}^{2}\right)}{\left(1+e_{2}^{2}\right)^{2}+2\left(1-e_{2}^{2}\right)} \\
\frac{\left(1-e_{2}^{2}\right)\left(1-2 e_{2}^{2}\right)}{\left(1-2 e_{2}^{2}\right)^{2}+2\left(1-e_{2}^{2}\right)}
\end{array}\right.
$$




$$
\begin{gathered}
\eta=-\frac{2}{3 h_{q}} \frac{\kappa Q^{*}(g+1)(g+f) \mathrm{sh}}{(g+1)^{2}+(g+f)^{2}+(g+1)(g+f)\left[\kappa H^{*} \mathrm{sh}-2 \mathrm{ch}\right]}, \\
C=-\frac{2}{3} \frac{\kappa(g+1)(g+f) \mathrm{sh}}{\left(Q^{*}+\frac{3}{2} h_{q} \eta H^{*}\right) \eta}, \quad \operatorname{sh} \equiv \sinh \left(\kappa H^{*}\right), \quad \operatorname{ch} \equiv \cosh \left(\kappa H^{*}\right)
\end{gathered}
$$

where $H^{*} \equiv 2 \sqrt{h_{q}}\left(\alpha_{1}-\alpha_{2}\right), Q^{*} \equiv \sqrt{h_{q}}(1-f)$ and $h_{q}$ is given by

$$
h_{q} \equiv \frac{2}{3} \boldsymbol{Q}: \hat{\mathrm{h}}: \boldsymbol{Q}=\frac{\hat{h}_{11}+\hat{h}_{22}+4 \hat{h}_{33}-4 \hat{h}_{23}-4 \hat{h}_{31}+2 \hat{h}_{12}}{6}
$$

Above, $H^{*}$ and $Q^{*}$ depend on an additional parameter $\alpha_{1}$, given by

$$
\alpha_{1}= \begin{cases}{\left[e_{1}-\left(1-e_{1}^{2}\right) \tanh ^{-1} e_{1}\right] /\left(2 e_{1}^{3}\right)} & (\mathrm{p}) \\ {\left[-e_{1}\left(1-e_{1}^{2}\right)+\sqrt{1-e_{1}^{2}} \sin ^{-1} e_{1}\right] /\left(2 e_{1}^{3}\right)} & (\mathrm{o})\end{cases}
$$

Note that $\alpha_{2}$ and $\alpha_{1}$ are identical to those given by [34] for isotropic matrices.

$$
\alpha_{1}^{\text {Gar }}= \begin{cases}\frac{1}{3-e_{1}^{2}} & (\mathrm{p}) \\ \frac{1-e_{1}^{2}}{3-2 e_{1}^{2}} & (\mathrm{o})\end{cases}
$$

Equation (A.8) was mistyped in Ref. [63]. The correct expressions given here correspond to those originally published in [39].

\section{Appendix B. Jacobian Matrix}

The components of the Jacobian matrix are given as follows, with $R_{A, B}=\frac{\partial R_{A}}{\partial B}$ and $\mathbf{0}$ is the second-rank zero tensor.

$$
\begin{aligned}
& \boldsymbol{R}_{\tilde{\boldsymbol{\sigma}}^{\prime}, \tilde{\boldsymbol{\sigma}}^{\prime}}= \frac{1}{2 \mu} \frac{1}{\Delta t} \mathbb{J} \\
&+\Lambda\left[3 C \frac{\mathbb{J}: \tilde{\mathbb{H}}: \mathbb{J}}{\bar{\sigma}^{2}}+2(g+1)(g+f) \frac{\kappa^{2}}{\bar{\sigma}^{2}} \cosh \left(\kappa \frac{\tilde{\boldsymbol{\sigma}}: \tilde{\boldsymbol{X}}}{\bar{\sigma}}\right)(\mathbb{J}: \tilde{\boldsymbol{X}}) \otimes(\mathbb{J}: \tilde{\boldsymbol{X}})\right] \\
& \boldsymbol{R}_{\tilde{\boldsymbol{\sigma}}^{\prime}, \tilde{\sigma}_{\mathrm{m}}}=\Lambda\left[3 C \frac{\mathbb{J}: \tilde{\mathbb{H}}: \boldsymbol{I}}{\bar{\sigma}^{2}}+2(g+1)(g+f) \frac{\kappa^{2}}{\bar{\sigma}^{2}} \cosh \left(\kappa \frac{\tilde{\boldsymbol{\sigma}}: \tilde{\boldsymbol{X}}}{\bar{\sigma}}\right)(\boldsymbol{I}: \tilde{\boldsymbol{X}})(\mathbb{J}: \tilde{\boldsymbol{X}})\right] \\
& \boldsymbol{R}_{\tilde{\boldsymbol{\sigma}}^{\prime}, f}=\Lambda\left[2(g+1) \kappa \sinh \left(\kappa \frac{\tilde{\boldsymbol{\sigma}}: \tilde{\boldsymbol{X}}}{\bar{\sigma}}\right) \frac{(\mathbb{J}: \tilde{\boldsymbol{X}})}{\bar{\sigma}}\right]
\end{aligned}
$$




$$
\begin{aligned}
& \boldsymbol{R}_{\tilde{\boldsymbol{\sigma}}^{\prime}, S}=\mathbf{0} \\
& \boldsymbol{R}_{\tilde{\boldsymbol{\sigma}}_{,}^{\prime}, \bar{\epsilon}}=\Lambda\left[3 C \mathbb{\mathbb { J }}: \tilde{\mathbb{H}}: \tilde{\boldsymbol{\sigma}}(-2) \bar{\sigma}^{-3} \frac{\partial \bar{\sigma}}{\partial \bar{\epsilon}}\right] \\
& -\Lambda\left[2(g+1)(g+f) \frac{\kappa^{2}}{\bar{\sigma}^{3}} \cosh \left(\kappa \frac{\tilde{\boldsymbol{\sigma}}: \tilde{\boldsymbol{X}}}{\bar{\sigma}}\right)(\tilde{\boldsymbol{\sigma}}: \tilde{\boldsymbol{X}}) \frac{\partial \bar{\sigma}}{\partial \bar{\epsilon}}(\mathbb{J}: \tilde{\boldsymbol{X}})\right] \\
& -\Lambda\left[2(g+1)(g+f) \frac{\kappa}{\bar{\sigma}^{2}} \sinh \left(\kappa \frac{\tilde{\boldsymbol{\sigma}}: \tilde{\boldsymbol{X}}}{\bar{\sigma}}\right) \frac{\partial \bar{\sigma}}{\partial \bar{\epsilon}}(\mathbb{\mathbb { J }}: \tilde{\boldsymbol{X}})\right], \\
& \text { where } \frac{\partial \sigma}{\partial \bar{\epsilon}}=\sigma_{\mathrm{S}} N\left(1+\frac{\bar{\epsilon}}{\epsilon_{0}}\right)^{N-1} \frac{1}{\epsilon_{0}} \\
& \boldsymbol{R}_{\tilde{\boldsymbol{\sigma}}^{\prime}, \Lambda}=\left[3 C \frac{\mathbb{\mathbb { J } :}: \tilde{\mathbb{H}}: \tilde{\boldsymbol{\sigma}}}{\bar{\sigma}^{2}}+2(g+1)(g+f) \kappa \sinh \left(\kappa \frac{\tilde{\boldsymbol{\sigma}}: \tilde{\boldsymbol{X}}}{\bar{\sigma}}\right) \frac{\mathbb{J}: \tilde{\boldsymbol{X}}}{\bar{\sigma}}\right] \\
& R_{\tilde{\sigma}_{\mathrm{m}}, \tilde{\boldsymbol{\sigma}}^{\prime}}=\Lambda\left[3 C \frac{\boldsymbol{I}: \tilde{\mathbb{H}}: \mathbb{I}}{\bar{\sigma}^{2}}+2(g+1)(g+f) \frac{\kappa^{2}}{\bar{\sigma}^{2}} \cosh \left(\kappa \frac{\tilde{\boldsymbol{\sigma}}: \tilde{\boldsymbol{X}}}{\bar{\sigma}}\right)(\boldsymbol{I}: \tilde{\boldsymbol{X}})(\mathbb{J}: \tilde{\boldsymbol{X}})\right] \\
& R_{\tilde{\sigma}_{\mathrm{m}}, \tilde{\sigma}_{\mathrm{m}}}=\frac{1}{K} \frac{1}{\Delta t}+\Lambda\left[3 C \frac{\boldsymbol{I}: \tilde{\mathbb{H}}: \boldsymbol{I}}{\bar{\sigma}^{2}}+2(g+1)(g+f) \frac{\kappa^{2}}{\bar{\sigma}^{2}} \cosh \left(\kappa \frac{\tilde{\boldsymbol{\sigma}}: \tilde{\boldsymbol{X}}}{\bar{\sigma}}\right)(\boldsymbol{I}: \tilde{\boldsymbol{X}})(\boldsymbol{I}: \tilde{\boldsymbol{X}})\right] \\
& R_{\tilde{\sigma}_{\mathrm{m}}, f}=\Lambda\left[2(g+1) \kappa \sinh \left(\kappa \frac{\tilde{\boldsymbol{\sigma}}: \tilde{\boldsymbol{X}}}{\bar{\sigma}}\right) \frac{(\boldsymbol{I}: \tilde{\boldsymbol{X}})}{\bar{\sigma}}\right] \\
& R_{\tilde{\sigma}_{\mathrm{m}}, S}=0 \\
& R_{\tilde{\sigma}_{\mathrm{m}}, \bar{\epsilon}}=\Lambda\left[3 C \boldsymbol{I}: \tilde{\mathbb{H}}: \tilde{\boldsymbol{\sigma}}(-2) \bar{\sigma}^{-3} \frac{\partial \bar{\sigma}}{\partial \bar{\epsilon}}\right] \\
& -\Lambda\left[2(g+1)(g+f) \frac{\kappa^{2}}{\bar{\sigma}^{3}} \cosh \left(\kappa \frac{\tilde{\boldsymbol{\sigma}}: \tilde{\boldsymbol{X}}}{\bar{\sigma}}\right)(\tilde{\boldsymbol{\sigma}}: \tilde{\boldsymbol{X}}) \frac{\partial \bar{\sigma}}{\partial \bar{\epsilon}}(\boldsymbol{I}: \tilde{\boldsymbol{X}})\right] \\
& -\Lambda\left[2(g+1)(g+f) \frac{\kappa}{\bar{\sigma}^{2}} \sinh \left(\kappa \frac{\tilde{\boldsymbol{\sigma}}: \tilde{\boldsymbol{X}}}{\bar{\sigma}}\right) \frac{\partial \bar{\sigma}}{\partial \bar{\epsilon}}(\boldsymbol{I}: \tilde{\boldsymbol{X}})\right] \\
& R_{\tilde{\sigma}_{\mathrm{m}}, \Lambda}=\left[3 C \frac{\boldsymbol{I}: \tilde{\mathbb{H}}: \tilde{\boldsymbol{\sigma}}}{\bar{\sigma}^{2}}+2(g+1)(g+f) \kappa \sinh \left(\kappa \frac{\tilde{\boldsymbol{\sigma}}: \tilde{\boldsymbol{X}}}{\bar{\sigma}}\right) \frac{\boldsymbol{I}: \tilde{\boldsymbol{X}}}{\bar{\sigma}}\right]
\end{aligned}
$$




$$
\begin{aligned}
& R_{f, \tilde{\boldsymbol{\sigma}}^{\prime}}=-(1-f) \Lambda\left[3 C \frac{\boldsymbol{I}: \tilde{\mathbb{H}}: \mathbb{\mathbb { I }}}{\bar{\sigma}^{2}}+2(g+1)(g+f) \frac{\kappa^{2}}{\bar{\sigma}^{2}} \cosh \left(\kappa \frac{\tilde{\boldsymbol{\sigma}}: \tilde{\boldsymbol{X}}}{\bar{\sigma}}\right)(\boldsymbol{I}: \tilde{\boldsymbol{X}})(\mathbb{I}: \tilde{\boldsymbol{X}})\right] \\
& R_{f, \tilde{\sigma}_{\mathrm{m}}}=-(1-f) \Lambda\left[3 C \frac{\boldsymbol{I}: \tilde{\mathbb{H}}: \boldsymbol{I}}{\bar{\sigma}^{2}}+2(g+1)(g+f) \frac{\kappa^{2}}{\bar{\sigma}^{2}} \cosh \left(\kappa \frac{\tilde{\boldsymbol{\sigma}}: \tilde{\boldsymbol{X}}}{\bar{\sigma}}\right)(\boldsymbol{I}: \tilde{\boldsymbol{X}})(\boldsymbol{I}: \tilde{\boldsymbol{X}})\right] \\
& R_{f, f}=\frac{1}{\Delta t}+\Lambda\left[3 C \frac{\boldsymbol{I}: \tilde{\mathbb{H}}: \tilde{\boldsymbol{\sigma}}}{\bar{\sigma}^{2}}+2(g+1)(g+f) \kappa \sinh \left(\kappa \frac{\tilde{\boldsymbol{\sigma}}: \tilde{\boldsymbol{X}}}{\bar{\sigma}}\right) \frac{\boldsymbol{I}: \tilde{\boldsymbol{X}}}{\bar{\sigma}}\right] \\
& -(1-f) \Lambda\left[2(g+1) \kappa \sinh \left(\kappa \frac{\tilde{\boldsymbol{\sigma}}: \tilde{\boldsymbol{X}}}{\bar{\sigma}}\right) \frac{(\boldsymbol{I}: \tilde{\boldsymbol{X}})}{\bar{\sigma}}\right] \\
& R_{f, S}=0 \\
& R_{f, \bar{\epsilon}}=-(1-f) \Lambda\left[3 C \boldsymbol{I}: \tilde{\mathbb{H}}: \tilde{\boldsymbol{\sigma}}(-2) \bar{\sigma}^{-3} \frac{\partial \bar{\sigma}}{\partial \bar{\epsilon}}\right] \\
& +(1-f) \Lambda\left[2(g+1)(g+f) \frac{\kappa^{2}}{\bar{\sigma}^{3}} \cosh \left(\kappa \frac{\tilde{\boldsymbol{\sigma}}: \tilde{\boldsymbol{X}}}{\bar{\sigma}}\right)(\tilde{\boldsymbol{\sigma}}: \tilde{\boldsymbol{X}}) \frac{\partial \bar{\sigma}}{\partial \bar{\epsilon}}(\boldsymbol{I}: \tilde{\boldsymbol{X}})\right] \\
& +(1-f) \Lambda\left[2(g+1)(g+f) \frac{\kappa}{\bar{\sigma}^{2}} \sinh \left(\kappa \frac{\tilde{\boldsymbol{\sigma}}: \tilde{\boldsymbol{X}}}{\bar{\sigma}}\right) \frac{\partial \bar{\sigma}}{\partial \bar{\epsilon}}(\boldsymbol{I}: \tilde{\boldsymbol{X}})\right] \\
& R_{f, \Lambda}=-(1-f)\left[3 C \frac{\boldsymbol{I}: \tilde{\mathbb{H}}: \tilde{\boldsymbol{\sigma}}}{\bar{\sigma}^{2}}+2(g+1)(g+f) \kappa \sinh \left(\kappa \frac{\tilde{\boldsymbol{\sigma}}: \tilde{\boldsymbol{X}}}{\bar{\sigma}}\right) \frac{\boldsymbol{I}: \tilde{\boldsymbol{X}}}{\bar{\sigma}}\right]
\end{aligned}
$$




$$
\begin{aligned}
& R_{S, \tilde{\boldsymbol{\sigma}}^{\prime}}=-3 k \Lambda C \frac{\tilde{\boldsymbol{Q}}: \tilde{\mathbb{H}}: \mathbb{\mathbb { I }}}{\bar{\sigma}^{2}}-2 k \Lambda(g+1)(g+f) \frac{\kappa^{2}}{\bar{\sigma}^{2}} \cosh \left(\kappa \frac{\tilde{\boldsymbol{\sigma}}: \tilde{\boldsymbol{X}}}{\bar{\sigma}}\right)(\tilde{\boldsymbol{Q}}: \tilde{\boldsymbol{X}})(\mathbb{\mathbb { J }}: \tilde{\boldsymbol{X}}) \\
& -\Lambda \tilde{\boldsymbol{Q}}:\left(\frac{1}{f} \tilde{\boldsymbol{X}}^{v}-\tilde{\boldsymbol{X}}\right)\left[3 C \frac{\boldsymbol{I}: \tilde{\mathbb{H}}: \mathbb{J}}{\bar{\sigma}^{2}}+2(g+1)(g+f) \frac{\kappa^{2}}{\bar{\sigma}^{2}} \cosh \left(\kappa \frac{\tilde{\boldsymbol{\sigma}}: \tilde{\boldsymbol{X}}}{\bar{\sigma}}\right)(\boldsymbol{I}: \tilde{\boldsymbol{X}})(\mathbb{\mathbb { J }}: \tilde{\boldsymbol{X}})\right] \\
& -\Lambda \tilde{\boldsymbol{Q}}:\left[3 C \frac{\tilde{\mathbb{H}}: \tilde{\boldsymbol{\sigma}}}{\bar{\sigma}^{2}}+2(g+1)(g+f) \kappa \sinh \left(\kappa \frac{\tilde{\boldsymbol{\sigma}}: \tilde{\boldsymbol{X}}}{\bar{\sigma}}\right) \frac{\tilde{\boldsymbol{X}}}{\bar{\sigma}}\right] \frac{\partial k}{\partial \tilde{\boldsymbol{\sigma}}^{\prime}} \\
& \text { where } \frac{\partial k}{\partial \tilde{\boldsymbol{\sigma}}^{\prime}}=\frac{9}{2} \frac{\left(\alpha_{1}-\alpha_{1}^{\mathrm{Gar}}\right)}{\left(1-3 \alpha_{1}\right)}(1-\sqrt{f})^{2} \frac{\partial k_{\mathcal{T}}}{\partial \tilde{\boldsymbol{\sigma}}^{\prime}}, \\
& \frac{\partial k_{\mathcal{T}}(\mathcal{T}, \epsilon)}{\partial \tilde{\boldsymbol{\sigma}}^{\prime}}=\left\{\begin{array}{l}
-\frac{\left(2 \mathcal{T}+4 \mathcal{T}^{3}\right)}{9} \frac{\partial \mathcal{T}}{\partial \tilde{\boldsymbol{\sigma}}^{\prime}} \text { for } \quad \epsilon=+1 \\
-\frac{\left(2 \mathcal{T}+4 \mathcal{T}^{3}\right)}{18} \frac{\partial \mathcal{T}}{\partial \tilde{\boldsymbol{\sigma}}^{\prime}} \text { for } \quad \epsilon=-1
\end{array}, \quad \epsilon \equiv \operatorname{sgn}\left(\tilde{\sigma}_{\mathrm{m}} \tilde{\sigma}_{33}^{\prime}\right)\right. \\
& \frac{\partial \mathcal{T}}{\partial \tilde{\boldsymbol{\sigma}}^{\prime}}=-\frac{3}{2} \tilde{\sigma}_{\mathrm{m}} \frac{\tilde{\boldsymbol{\sigma}}^{\prime}}{\left(\frac{3}{2} \tilde{\boldsymbol{\sigma}}^{\prime}: \tilde{\boldsymbol{\sigma}}^{\prime}\right)^{\frac{3}{2}}} \\
& R_{S, \tilde{\sigma}_{\mathrm{m}}}=-3 k \Lambda C \frac{\tilde{\boldsymbol{Q}}: \tilde{\mathbb{H}}: \boldsymbol{I}}{\bar{\sigma}^{2}}-2 k \Lambda(g+1)(g+f) \frac{\kappa^{2}}{\bar{\sigma}^{2}} \cosh \left(\kappa \frac{\tilde{\boldsymbol{\sigma}}: \tilde{\boldsymbol{X}}}{\bar{\sigma}}\right)(\tilde{\boldsymbol{Q}}: \tilde{\boldsymbol{X}})(\boldsymbol{I}: \tilde{\boldsymbol{X}}) \\
& -\Lambda \tilde{\boldsymbol{Q}}:\left(\frac{1}{f} \tilde{\boldsymbol{X}}^{v}-\tilde{\boldsymbol{X}}\right)\left[3 C \frac{\boldsymbol{I}: \tilde{\mathbb{H}}: \boldsymbol{I}}{\bar{\sigma}^{2}}+2(g+1)(g+f) \frac{\kappa^{2}}{\bar{\sigma}^{2}} \cosh \left(\kappa \frac{\tilde{\boldsymbol{\sigma}}: \tilde{\boldsymbol{X}}}{\bar{\sigma}}\right)(\boldsymbol{I}: \tilde{\boldsymbol{X}})(\boldsymbol{I}: \tilde{\boldsymbol{X}})\right] \\
& -\Lambda \tilde{\boldsymbol{Q}}:\left[3 C \frac{\tilde{\mathbb{H}}: \tilde{\boldsymbol{\sigma}}}{\bar{\sigma}^{2}}+2(g+1)(g+f) \kappa \sinh \left(\kappa \frac{\tilde{\boldsymbol{\sigma}}: \tilde{\boldsymbol{X}}}{\bar{\sigma}}\right) \frac{\tilde{\boldsymbol{X}}}{\bar{\sigma}}\right] \frac{\partial k}{\partial \tilde{\sigma}_{\mathrm{m}}} \\
& \text { where } \frac{\partial k}{\partial \tilde{\sigma}_{\mathrm{m}}}=\frac{9}{2} \frac{\left(\alpha_{1}-\alpha_{1}^{\mathrm{Gar}}\right)}{\left(1-3 \alpha_{1}\right)}(1-\sqrt{f})^{2} \frac{\partial k_{\mathcal{T}}}{\partial \tilde{\sigma}_{\mathrm{m}}}, \\
& \frac{\partial k_{\mathcal{T}}(\mathcal{T}, \epsilon)}{\partial \tilde{\sigma}_{\mathrm{m}}}=\left\{\begin{array}{l}
-\frac{\left(2 \mathcal{T}+4 \mathcal{T}^{3}\right)}{9} \frac{\partial \mathcal{T}}{\partial \tilde{\sigma}_{\mathrm{m}}} \text { for } \quad \epsilon=+1 \\
-\frac{\left(2 \mathcal{T}+4 \mathcal{T}^{3}\right)}{18} \frac{\partial \mathcal{T}}{\partial \tilde{\sigma}_{\mathrm{m}}} \text { for } \quad \epsilon=-1
\end{array}, \quad \epsilon \equiv \operatorname{sgn}\left(\tilde{\sigma}_{\mathrm{m}} \tilde{\sigma}_{33}^{\prime}\right)\right. \\
& \frac{\partial \mathcal{T}}{\partial \tilde{\sigma}_{\mathrm{m}}}=\left(\frac{3}{2} \tilde{\boldsymbol{\sigma}}^{\prime}: \tilde{\boldsymbol{\sigma}}^{\prime}\right)^{-\frac{1}{2}}
\end{aligned}
$$




$$
\begin{aligned}
& R_{S, f}=-2 k \Lambda(g+1) \kappa \sinh \left(\kappa \frac{\tilde{\boldsymbol{\sigma}}: \tilde{\boldsymbol{X}}}{\bar{\sigma}}\right)\left(\frac{\tilde{\boldsymbol{Q}}: \tilde{\boldsymbol{X}}}{\bar{\sigma}}\right) \\
& -\Lambda \tilde{\boldsymbol{Q}}:\left((-1) f^{-2} \tilde{\boldsymbol{X}}^{v}\right)\left[3 C \frac{\boldsymbol{I}: \tilde{\mathbb{H}}: \tilde{\boldsymbol{\sigma}}}{\bar{\sigma}^{2}}+2(g+1)(g+f) \kappa \sinh \left(\kappa \frac{\tilde{\boldsymbol{\sigma}}: \tilde{\boldsymbol{X}}}{\bar{\sigma}}\right) \frac{\boldsymbol{I}: \tilde{\boldsymbol{X}}}{\bar{\sigma}}\right] \\
& -\Lambda \tilde{\boldsymbol{Q}}:\left(\frac{1}{f} \tilde{\boldsymbol{X}}^{v}-\tilde{\boldsymbol{X}}\right)\left[2(g+1) \frac{\kappa}{\bar{\sigma}} \sinh \left(\kappa \frac{\tilde{\boldsymbol{\sigma}}: \tilde{\boldsymbol{X}}}{\bar{\sigma}}\right)(\boldsymbol{I}: \tilde{\boldsymbol{X}})\right] \\
& -\Lambda \tilde{\boldsymbol{Q}}:\left[3 C \frac{\tilde{\mathbb{H}}: \tilde{\boldsymbol{\sigma}}}{\bar{\sigma}^{2}}+2(g+1)(g+f) \kappa \sinh \left(\kappa \frac{\tilde{\boldsymbol{\sigma}}: \tilde{\boldsymbol{X}}}{\bar{\sigma}}\right) \frac{\tilde{\boldsymbol{X}}}{\bar{\sigma}}\right] \frac{\partial k}{\partial f}, \\
& \text { where } \frac{\partial k}{\partial f}=\frac{9}{2} \frac{\left(\alpha_{1}-\alpha_{1}^{\mathrm{Gar}}\right)}{\left(1-3 \alpha_{1}\right)} 2(1-\sqrt{f})(-1) \frac{1}{2} f^{-\frac{1}{2}} k_{\mathcal{T}} \\
& R_{S, S}=\frac{1}{\Delta t} \\
& R_{S, \bar{\epsilon}}=-3 k \Lambda C \tilde{\boldsymbol{Q}}: \tilde{\mathbb{H}}: \tilde{\boldsymbol{\sigma}}(-2) \bar{\sigma}^{-3} \frac{\partial \bar{\sigma}}{\partial \bar{\epsilon}} \\
& -2 k \Lambda(\tilde{\boldsymbol{Q}}: \tilde{\boldsymbol{X}})(g+1)(g+f) \kappa\left[\cosh \left(\kappa \frac{\tilde{\boldsymbol{\sigma}}: \tilde{\boldsymbol{X}}}{\bar{\sigma}}\right) \kappa(\tilde{\boldsymbol{\sigma}}: \tilde{\boldsymbol{X}})(-1) \bar{\sigma}^{-3} \frac{\partial \bar{\sigma}}{\partial \bar{\epsilon}}\right] \\
& -2 k \Lambda(\tilde{\boldsymbol{Q}}: \tilde{\boldsymbol{X}})(g+1)(g+f) \kappa\left[\sinh \left(\kappa \frac{\tilde{\boldsymbol{\sigma}}: \tilde{\boldsymbol{X}}}{\bar{\sigma}}\right)(-1) \bar{\sigma}^{-2} \frac{\partial \bar{\sigma}}{\partial \bar{\epsilon}}\right] \\
& -\Lambda \tilde{\boldsymbol{Q}}:\left(\frac{1}{f} \tilde{\boldsymbol{X}}^{v}-\tilde{\boldsymbol{X}}\right)\left[3 C(\boldsymbol{I}: \tilde{\mathbb{H}}: \tilde{\boldsymbol{\sigma}})(-2) \bar{\sigma}^{-3} \frac{\partial \bar{\sigma}}{\partial \bar{\epsilon}}\right] \\
& -\Lambda \tilde{\boldsymbol{Q}}:\left(\frac{1}{f} \tilde{\boldsymbol{X}}^{v}-\tilde{\boldsymbol{X}}\right)\left[2(g+1)(g+f)(-1) \frac{\kappa^{2}}{\bar{\sigma}^{3}} \cosh \left(\kappa \frac{\tilde{\boldsymbol{\sigma}}: \tilde{\boldsymbol{X}}}{\bar{\sigma}}\right)(\tilde{\boldsymbol{\sigma}}: \tilde{\boldsymbol{X}})(\boldsymbol{I}: \tilde{\boldsymbol{X}}) \frac{\partial \bar{\sigma}}{\partial \bar{\epsilon}}\right] \\
& -\Lambda \tilde{\boldsymbol{Q}}:\left(\frac{1}{f} \tilde{\boldsymbol{X}}^{v}-\tilde{\boldsymbol{X}}\right)\left[2(g+1)(g+f)(-1) \frac{\kappa}{\bar{\sigma}^{2}} \sinh \left(\kappa \frac{\tilde{\boldsymbol{\sigma}}: \tilde{\boldsymbol{X}}}{\bar{\sigma}}\right)(\boldsymbol{I}: \tilde{\boldsymbol{X}}) \frac{\partial \bar{\sigma}}{\partial \bar{\epsilon}}\right] \\
& R_{S, \Lambda}=-k \tilde{\boldsymbol{Q}}:\left[3 C \frac{\tilde{\mathbb{H}}: \tilde{\boldsymbol{\sigma}}}{\bar{\sigma}^{2}}+2(g+1)(g+f) \kappa \sinh \left(\kappa \frac{\tilde{\boldsymbol{\sigma}}: \tilde{\boldsymbol{X}}}{\bar{\sigma}}\right) \frac{\tilde{\boldsymbol{X}}}{\bar{\sigma}}\right] \\
& -\tilde{\boldsymbol{Q}}:\left(\frac{1}{f} \tilde{\boldsymbol{X}}^{v}-\tilde{\boldsymbol{X}}\right)\left[3 C \frac{\boldsymbol{I}: \tilde{\mathbb{H}}: \tilde{\boldsymbol{\sigma}}}{\bar{\sigma}^{2}}+2(g+1)(g+f) \kappa \sinh \left(\kappa \frac{\tilde{\boldsymbol{\sigma}}: \tilde{\boldsymbol{X}}}{\bar{\sigma}}\right) \frac{\boldsymbol{I}: \tilde{\boldsymbol{X}}}{\bar{\sigma}}\right]
\end{aligned}
$$




$$
\begin{aligned}
& R_{\bar{\epsilon}, \tilde{\boldsymbol{\sigma}}^{\prime}}=-\frac{1}{(1-f)} \Lambda\left[3 C \frac{2 \tilde{\boldsymbol{\sigma}}: \tilde{\mathbb{H}}: \mathbb{\mathbb { I }}}{\bar{\sigma}^{3}}+2(g+1)(g+f) \frac{\kappa^{2}}{\bar{\sigma}^{3}} \cosh \left(\kappa \frac{\tilde{\boldsymbol{\sigma}}: \tilde{\boldsymbol{X}}}{\bar{\sigma}}\right)(\tilde{\boldsymbol{\sigma}}: \tilde{\boldsymbol{X}})(\mathbb{\mathbb { J }}: \tilde{\boldsymbol{X}})\right] \\
& -\frac{1}{(1-f)} \Lambda\left[2(g+1)(g+f) \frac{\kappa}{\bar{\sigma}^{2}} \sinh \left(\kappa \frac{\tilde{\boldsymbol{\sigma}}: \tilde{\boldsymbol{X}}}{\bar{\sigma}}\right)(\mathbb{\mathbb { J }}: \tilde{\boldsymbol{X}})\right]
\end{aligned}
$$

$$
\begin{aligned}
& R_{\bar{\epsilon}, \tilde{\sigma}_{\mathrm{m}}}=-\frac{1}{(1-f)} \Lambda\left[3 C \frac{2 \tilde{\boldsymbol{\sigma}}: \tilde{\mathbb{H}}: \boldsymbol{I}}{\bar{\sigma}^{3}}+2(g+1)(g+f) \frac{\kappa^{2}}{\bar{\sigma}^{3}} \cosh \left(\kappa \frac{\tilde{\boldsymbol{\sigma}}: \tilde{\boldsymbol{X}}}{\bar{\sigma}}\right)(\tilde{\boldsymbol{\sigma}}: \tilde{\boldsymbol{X}})(\boldsymbol{I}: \tilde{\boldsymbol{X}})\right] \\
& -\frac{1}{(1-f)} \Lambda\left[2(g+1)(g+f) \frac{\kappa}{\bar{\sigma}^{2}} \sinh \left(\kappa \frac{\tilde{\boldsymbol{\sigma}}: \tilde{\boldsymbol{X}}}{\bar{\sigma}}\right)(\boldsymbol{I}: \tilde{\boldsymbol{X}})\right]
\end{aligned}
$$

$$
\begin{gathered}
R_{\bar{\epsilon}, f}=-\frac{1}{(1-f)^{2}} \Lambda\left[3 C \frac{\tilde{\boldsymbol{\sigma}}: \tilde{\mathbb{H}}: \tilde{\boldsymbol{\sigma}}}{\bar{\sigma}^{3}}+2(g+1)(g+f) \frac{\kappa}{\bar{\sigma}^{2}} \sinh \left(\kappa \frac{\tilde{\boldsymbol{\sigma}}: \tilde{\boldsymbol{X}}}{\bar{\sigma}}\right)(\tilde{\boldsymbol{\sigma}}: \tilde{\boldsymbol{X}})\right] \\
-\frac{1}{(1-f)} \Lambda\left[2(g+1) \frac{\kappa}{\bar{\sigma}^{2}} \sinh \left(\kappa \frac{\tilde{\boldsymbol{\sigma}}: \tilde{\boldsymbol{X}}}{\bar{\sigma}}\right)(\tilde{\boldsymbol{\sigma}}: \tilde{\boldsymbol{X}})\right] \\
R_{\bar{\epsilon}, S}=0
\end{gathered}
$$

$$
\begin{aligned}
R_{\bar{\epsilon}, \bar{\epsilon}}= & \frac{1}{\Delta t}-\frac{1}{(1-f)} \Lambda\left[3 C \tilde{\boldsymbol{\sigma}}: \tilde{\mathbb{H}}: \tilde{\boldsymbol{\sigma}}(-3) \bar{\sigma}^{-4} \frac{\partial \bar{\sigma}}{\partial \bar{\epsilon}}\right] \\
& +\frac{1}{(1-f)} \Lambda\left[2(g+1)(g+f) \frac{\kappa^{2}}{\bar{\sigma}^{4}} \cosh \left(\kappa \frac{\tilde{\boldsymbol{\sigma}}: \tilde{\boldsymbol{X}}}{\bar{\sigma}}\right)(\tilde{\boldsymbol{\sigma}}: \tilde{\boldsymbol{X}}) \frac{\partial \bar{\sigma}}{\partial \bar{\epsilon}}(\tilde{\boldsymbol{\sigma}}: \tilde{\boldsymbol{X}})\right] \\
& +\frac{1}{(1-f)} \Lambda\left[4(g+1)(g+f) \frac{\kappa}{\bar{\sigma}^{3}} \sinh \left(\kappa \frac{\tilde{\boldsymbol{\sigma}}: \tilde{\boldsymbol{X}}}{\bar{\sigma}}\right) \frac{\partial \bar{\sigma}}{\partial \bar{\epsilon}}(\tilde{\boldsymbol{\sigma}}: \tilde{\boldsymbol{X}})\right] \\
R_{\bar{\epsilon}, \Lambda}= & -\frac{1}{(1-f)}\left[3 C \frac{\tilde{\boldsymbol{\sigma}}: \tilde{\mathbb{H}}: \tilde{\boldsymbol{\sigma}}}{\bar{\sigma}^{3}}+2(g+1)(g+f) \kappa \sinh \left(\kappa \frac{\tilde{\boldsymbol{\sigma}}: \tilde{\boldsymbol{X}}}{\bar{\sigma}}\right) \frac{\tilde{\boldsymbol{\sigma}}: \tilde{\boldsymbol{X}}}{\bar{\sigma}^{2}}\right] \\
& R_{\Lambda, \tilde{\boldsymbol{\sigma}}^{\prime}=}=C \frac{3 \tilde{\boldsymbol{\sigma}}: \tilde{\mathbb{H}}: \mathbb{\mathbb { J }}}{\bar{\sigma}^{2}}+2(g+1)(g+f) \frac{\kappa}{\bar{\sigma}} \sinh \left(\kappa \frac{\tilde{\boldsymbol{\sigma}}: \tilde{\boldsymbol{X}}}{\bar{\sigma}}\right)(\mathbb{J}: \tilde{\boldsymbol{X}}) \\
& R_{\Lambda, \tilde{\sigma}_{\mathrm{m}}}=C \frac{3 \tilde{\boldsymbol{\sigma}}: \tilde{\mathbb{H}}: \boldsymbol{I}}{\bar{\sigma}^{2}}+2(g+1)(g+f) \frac{\kappa}{\bar{\sigma}} \sinh \left(\kappa \frac{\tilde{\boldsymbol{\sigma}}: \tilde{\boldsymbol{X}}}{\bar{\sigma}}\right)(\boldsymbol{I}: \tilde{\boldsymbol{X}})
\end{aligned}
$$




$$
\begin{aligned}
& R_{\Lambda, f}=2(g+1) \cosh \left(\kappa \frac{\tilde{\boldsymbol{\sigma}}: \tilde{\boldsymbol{X}}}{\bar{\sigma}}\right)-2(g+f) \\
& R_{\Lambda, S}=0 \\
& R_{\Lambda, \bar{\epsilon}}=C \frac{3}{2} \tilde{\boldsymbol{\sigma}}: \tilde{\mathbb{H}}: \tilde{\boldsymbol{\sigma}}(-2) \bar{\sigma}^{-3} \frac{\partial \bar{\sigma}}{\partial \bar{\epsilon}} \\
& -2(g+1)(g+f) \frac{\kappa}{\bar{\sigma}^{2}} \sinh \left(\kappa \frac{\tilde{\boldsymbol{\sigma}}: \tilde{\boldsymbol{X}}}{\bar{\sigma}}\right) \frac{\partial \bar{\sigma}}{\partial \bar{\epsilon}}(\tilde{\boldsymbol{\sigma}}: \tilde{\boldsymbol{X}}) \\
& R_{\Lambda, \Lambda}=0 \\
& \boldsymbol{R}_{\tilde{\boldsymbol{\sigma}}^{\prime}, \tilde{D}}=-\mathbb{J} \\
& R_{\tilde{\sigma}_{\mathrm{m}}, \tilde{\boldsymbol{D}}}=-\boldsymbol{I} \\
& R_{f, \tilde{D}}=R_{S, \tilde{D}}=R_{\bar{\epsilon}, \tilde{D}}=R_{\Lambda, \tilde{D}}=\mathbf{0}
\end{aligned}
$$


Table 1: The matrix anisotropy coefficients, $h_{i}$, used in the calculations, expressed in the basis of material orthotropy $\left(\underline{e}_{\mathrm{L}}, \underline{e}_{\mathrm{T}}, \underline{e}_{\mathrm{S}}\right)$.

\begin{tabular}{lcccccc}
\hline & $h_{\mathrm{L}}$ & $h_{\mathrm{T}}$ & $h_{\mathrm{S}}$ & $h_{\mathrm{TS}}$ & $h_{\mathrm{SL}}$ & $h_{\mathrm{LT}}$ \\
\hline Isotropic & 1.000 & 1.000 & 1.000 & 1.000 & 1.000 & 1.000 \\
\hline MAT1 & 1.000 & 1.000 & 1.000 & 2.333 & 2.333 & 1.000 \\
\hline MAT2 & 1.000 & 1.000 & 1.000 & 0.500 & 0.500 & 1.000 \\
\hline MAT3 & 1.650 & 0.778 & 0.893 & 1.378 & 0.943 & 1.627 \\
\hline
\end{tabular}




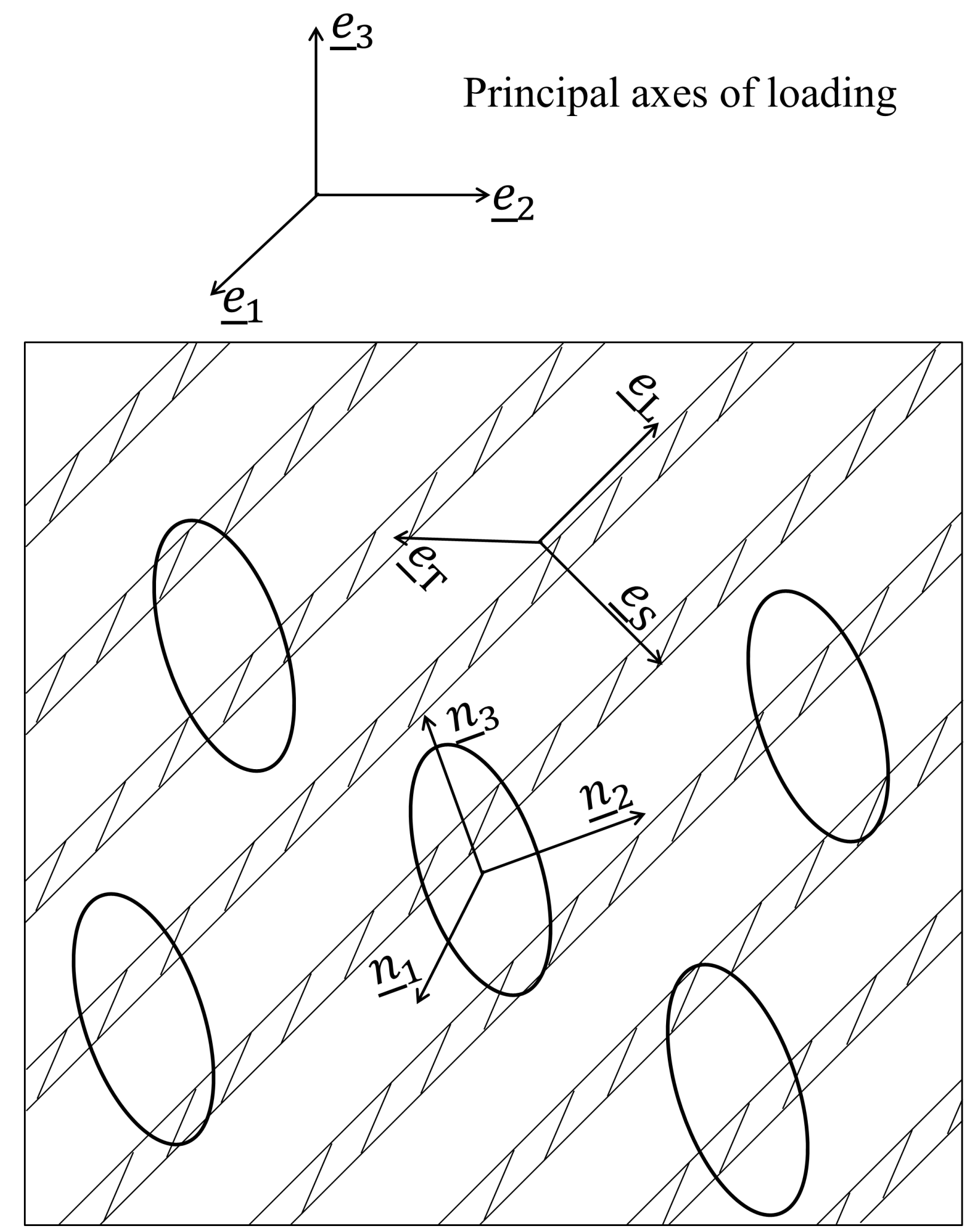

Figure 1: Schematic diagram of microstructure that consists of an aggregate of aligned spheroidal voids surrounded by the anisotropic matrix. 


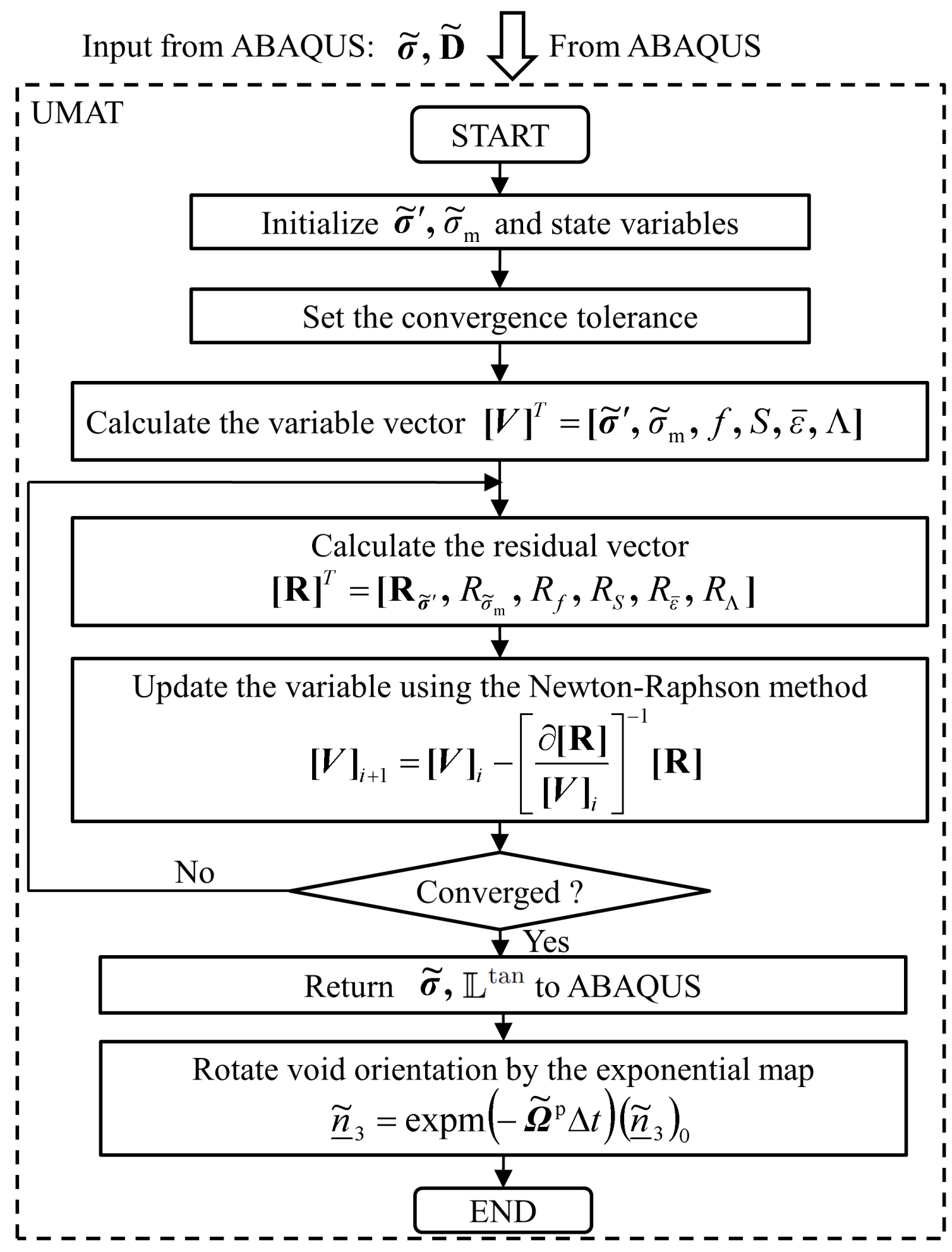

Output to ABAQUS: $\widetilde{\boldsymbol{\sigma}}, \mathbb{L}^{\tan } \square$ To ABAQUS

Figure 2: Flowchart of the integration algorithm in the user-defined material subroutine (UMAT). 
(a)
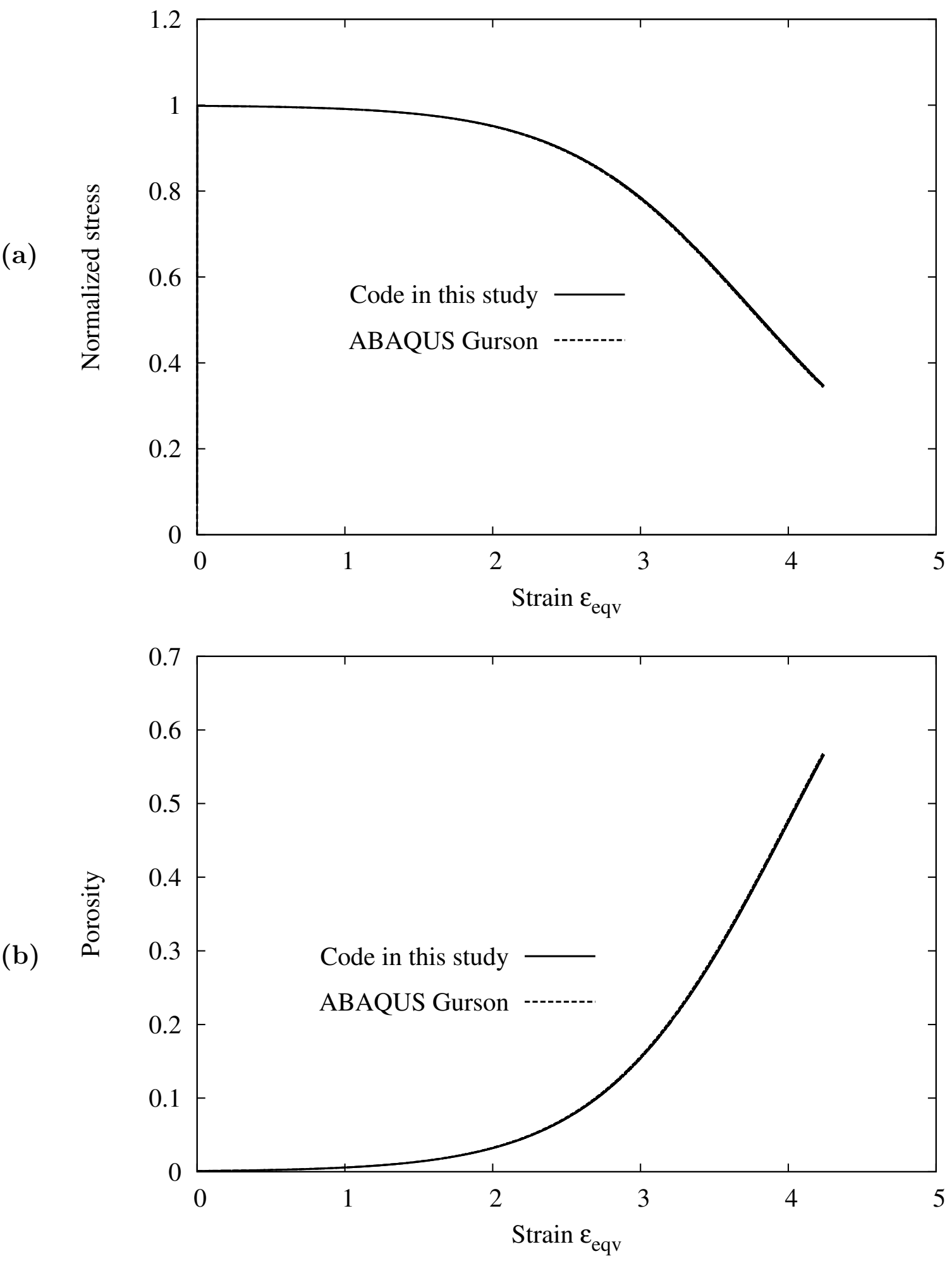

Figure 3: Illustration of code verification against the Gurson model from the ABAQUS standard library for biaxial tension of isotropic nonhardening matrix containing spherical voids $\left(f_{0}=0.0001, w=1\right)$. (a) Normalized stress $\sigma_{\text {eqv }} / \bar{\sigma}$ versus equivalent strain $\varepsilon_{\text {eqv }}$ in (49). (b) Porosity versus $\varepsilon_{\text {eqv }}$. 


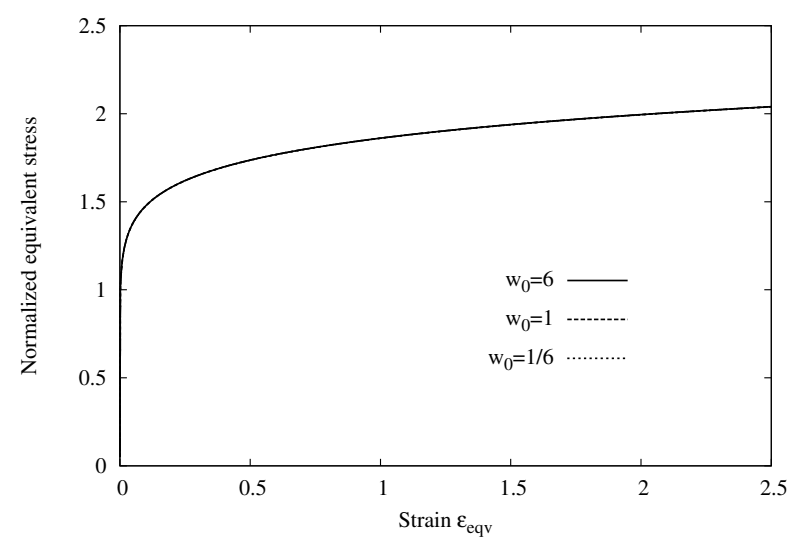

(a)

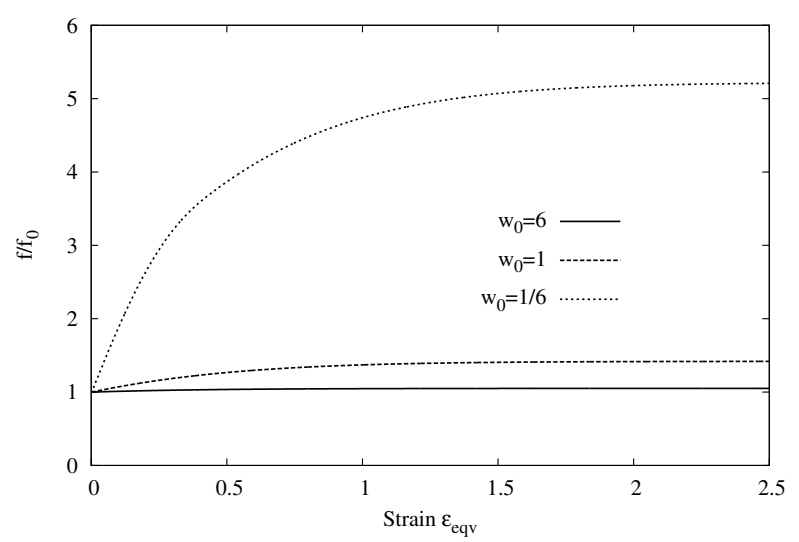

(c)

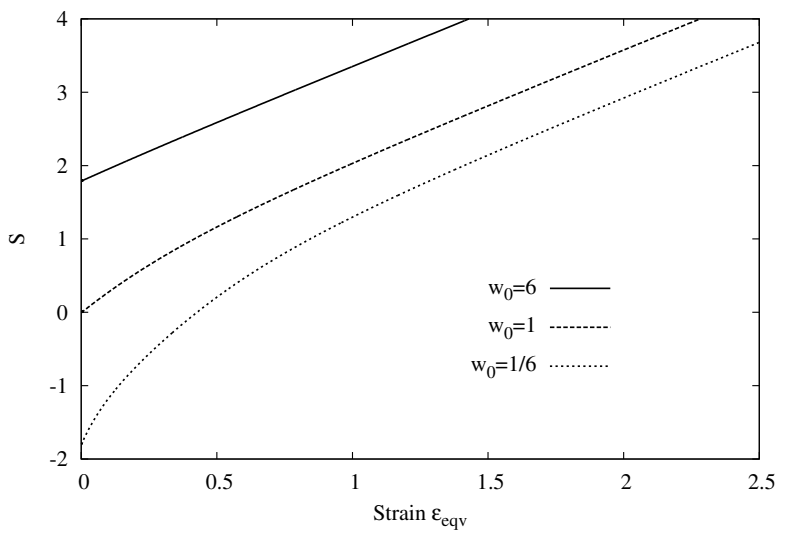

(e)

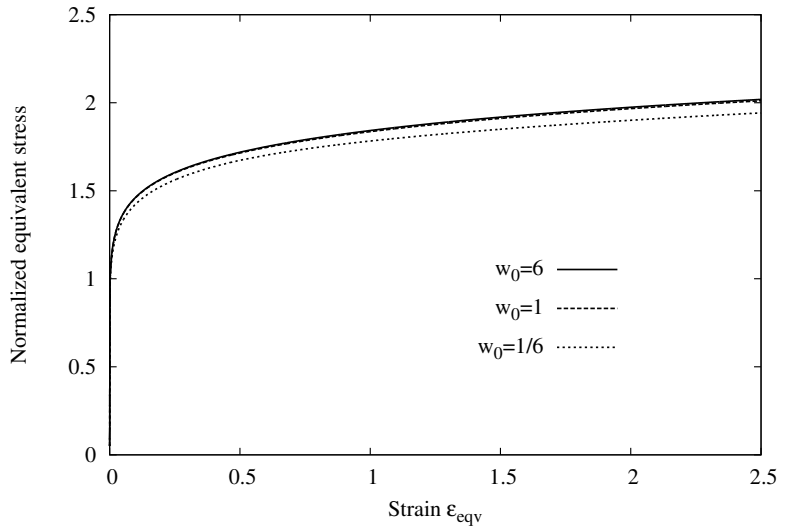

(b)

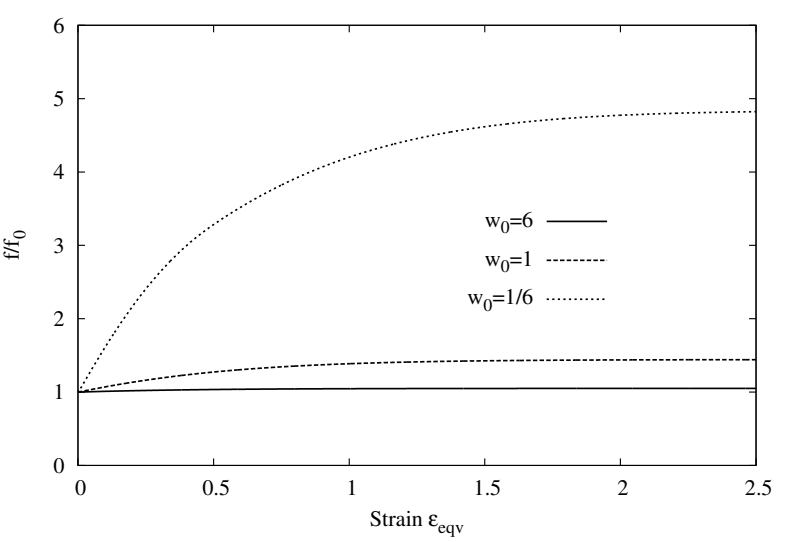

(d)

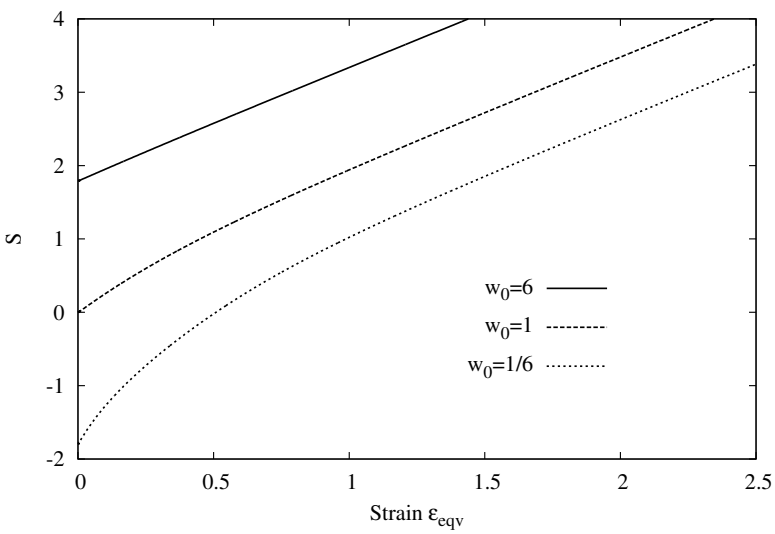

(f)

Figure 4: Parallel loading to the void main axis for $\mathcal{T}=1 / 3$. Spheroidal void in the isotropic matrix. Left plots (a), (c) and (e) are for $f_{0}=0.0001$ and right plots (b), (d) and (f) are for $f_{0}=0.01$. (a)(b) Normalized stress, (c)(d) normalized porosity $f / f_{0}$, (e)(f) void aspect ratio $S=\ln w$. 


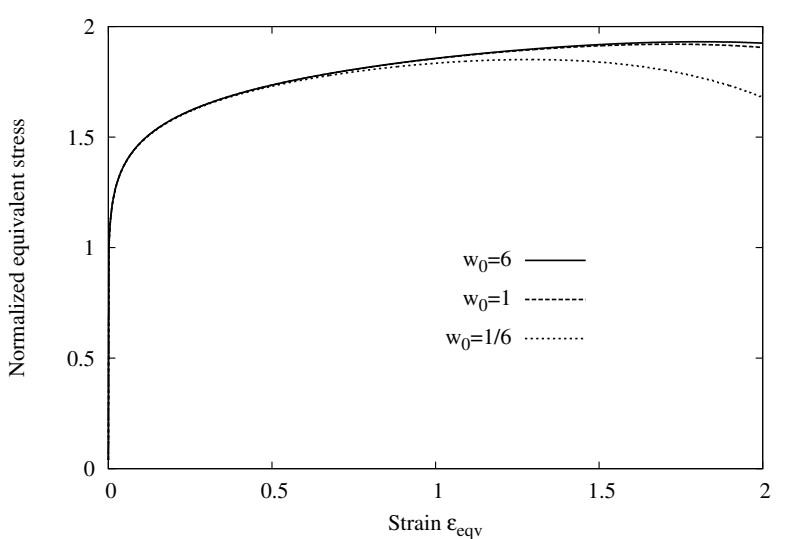

(a)

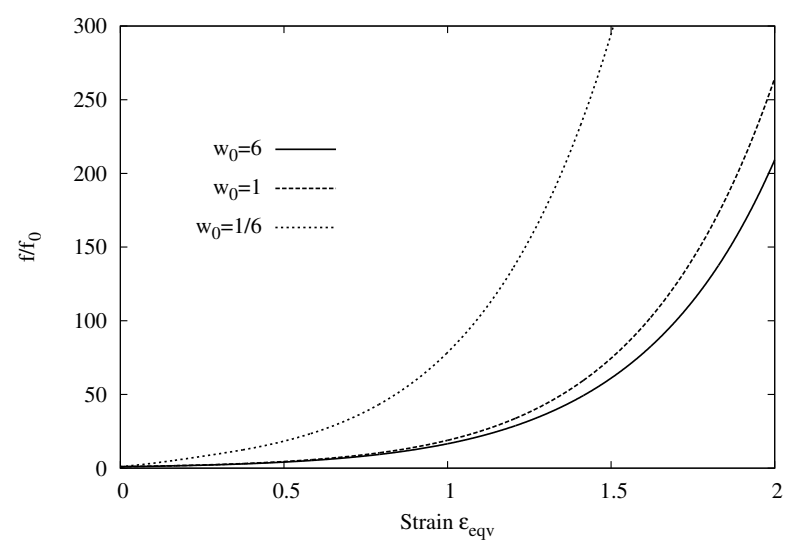

(c)

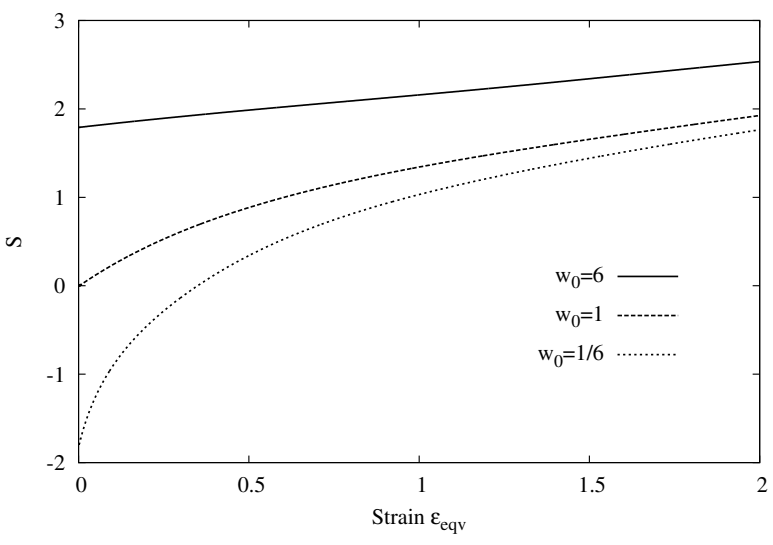

(e)

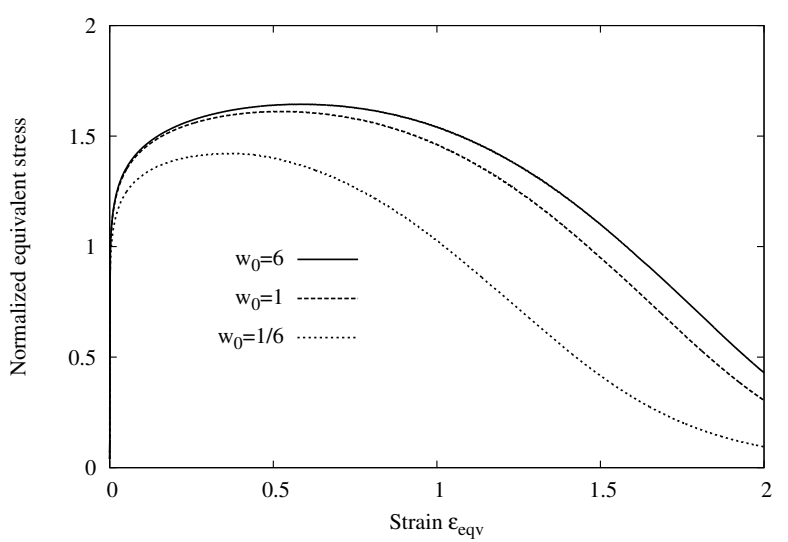

(b)

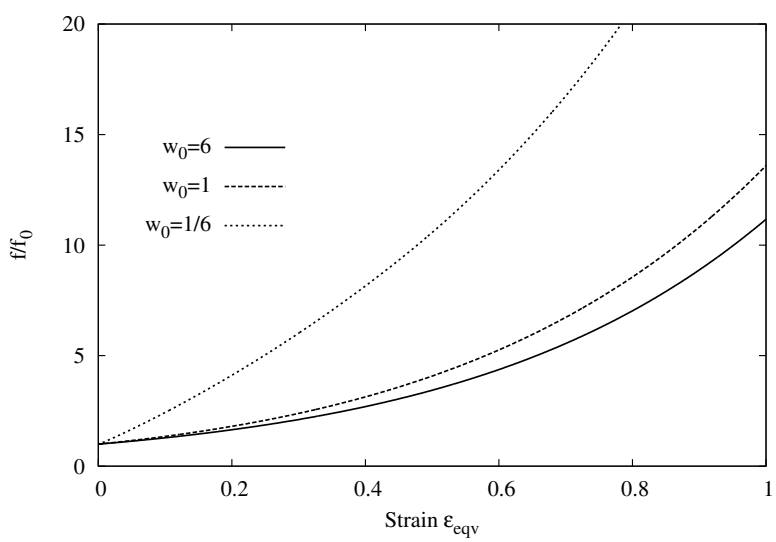

(d)

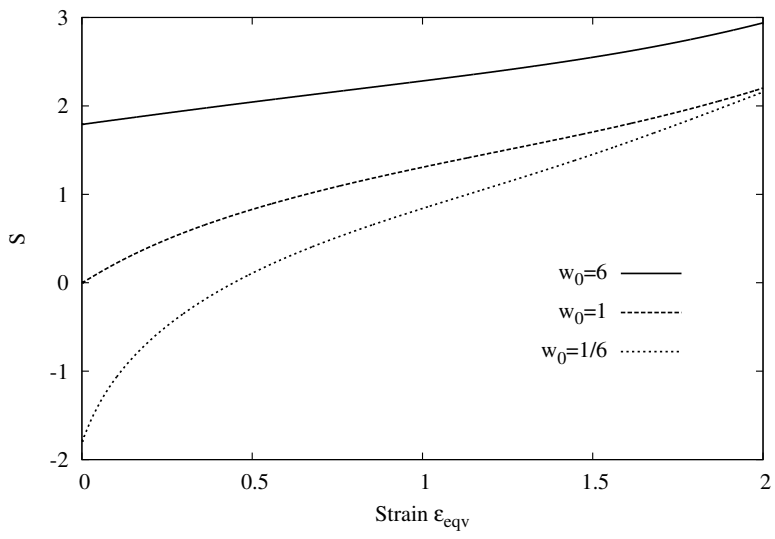

(f)

Figure 5: Parallel loading to the void main axis for $\mathcal{T}=1$. Spheroidal void in the isotropic matrix. Left plots (a), (c) and (e) are for $f_{0}=0.0001$ and right plots (b), (d) and (f) are for $f_{0}=0.01$. (a)(b) Normalized stress, (c)(d) normalized porosity $f / f_{0}$, (e)(f) void aspect ratio $S=\ln w$. 


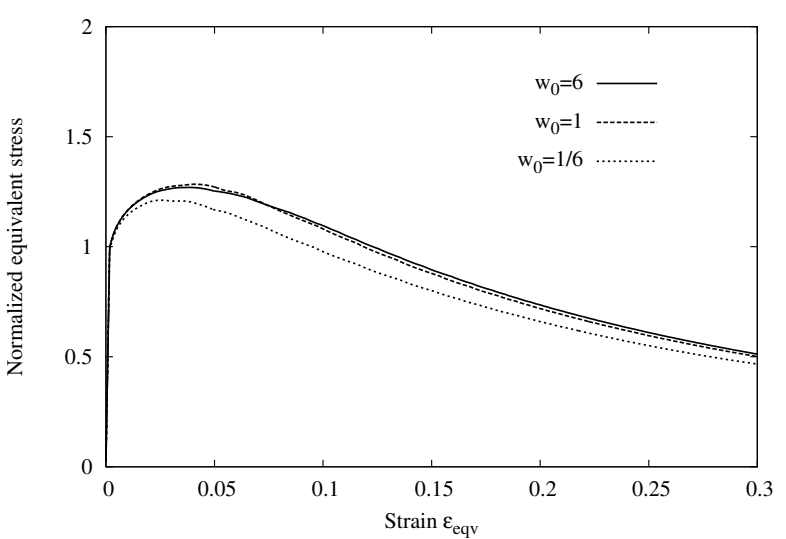

(a)

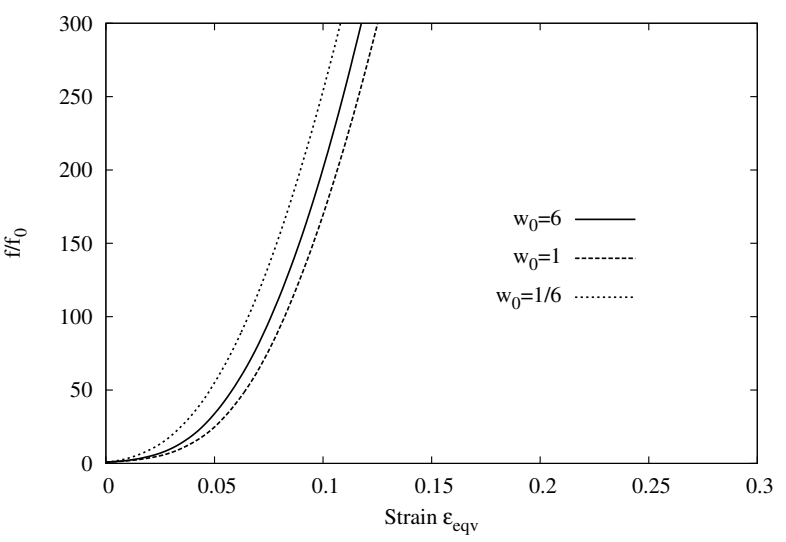

(c)

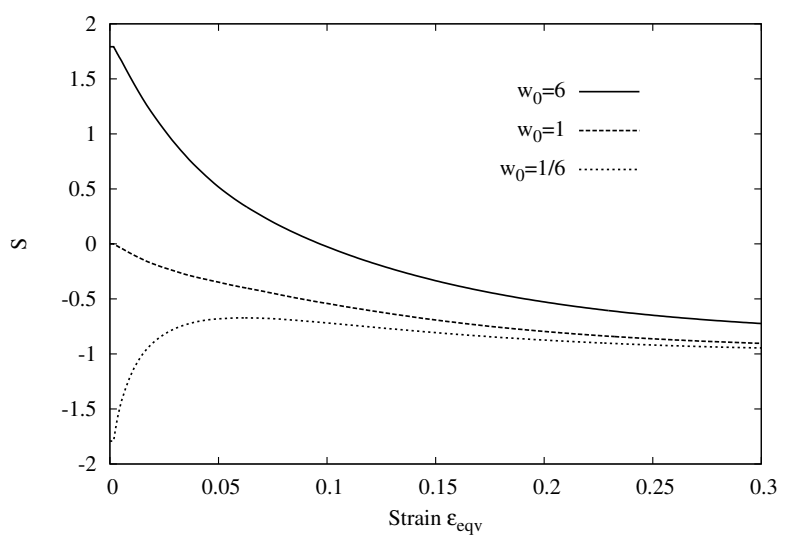

(e)

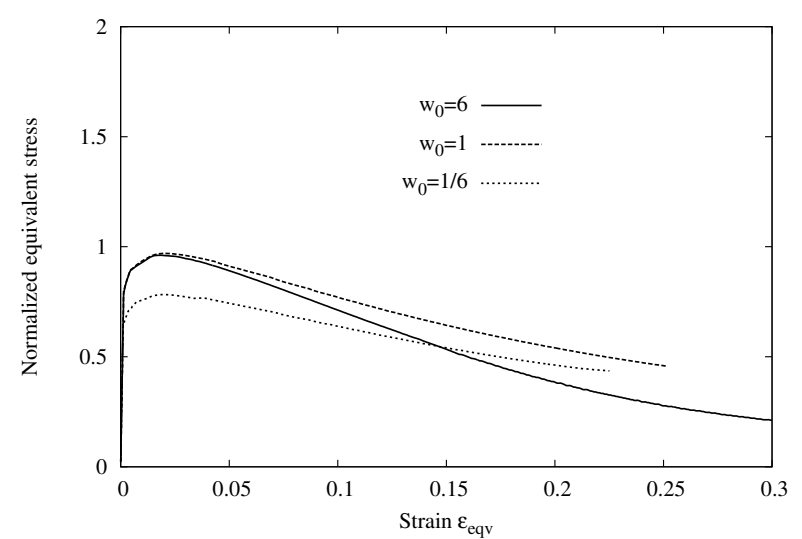

(b)

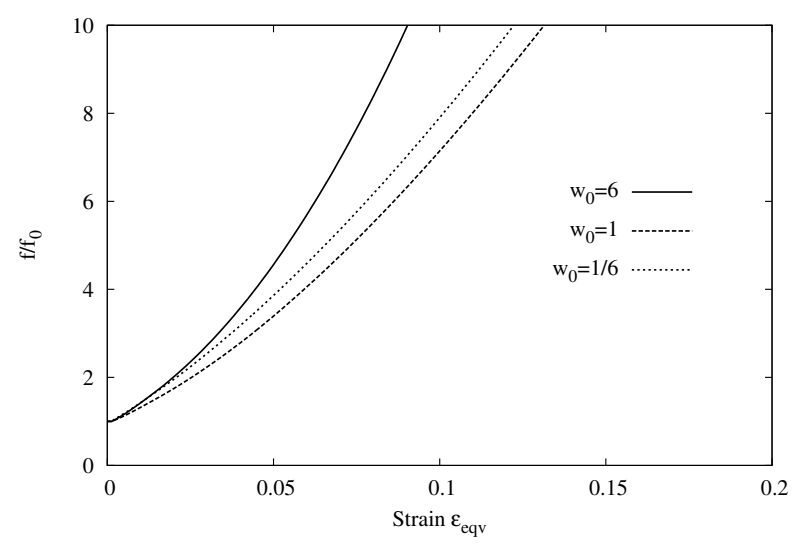

(d)

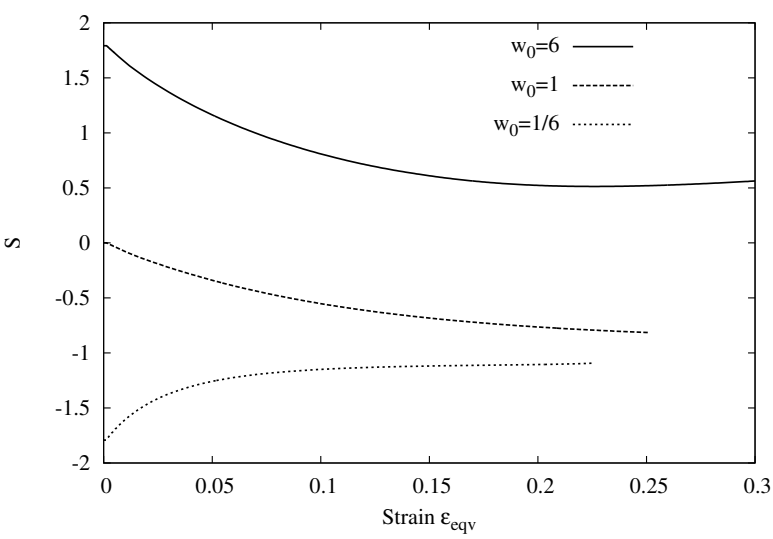

(f)

Figure 6: Parallel loading to the void main axis for $\mathcal{T}=3$. Spheroidal void in the isotropic matrix. The left plots (a), (c) and (e) are for $f_{0}=0.0001$ and the right plots (b), (d) and (f) are for $f_{0}=0.01$. (a)(b) Normalized stress, (c)(d) normalized porosity $f / f_{0}$, (e)(f) void aspect ratio $S=\ln w$. 

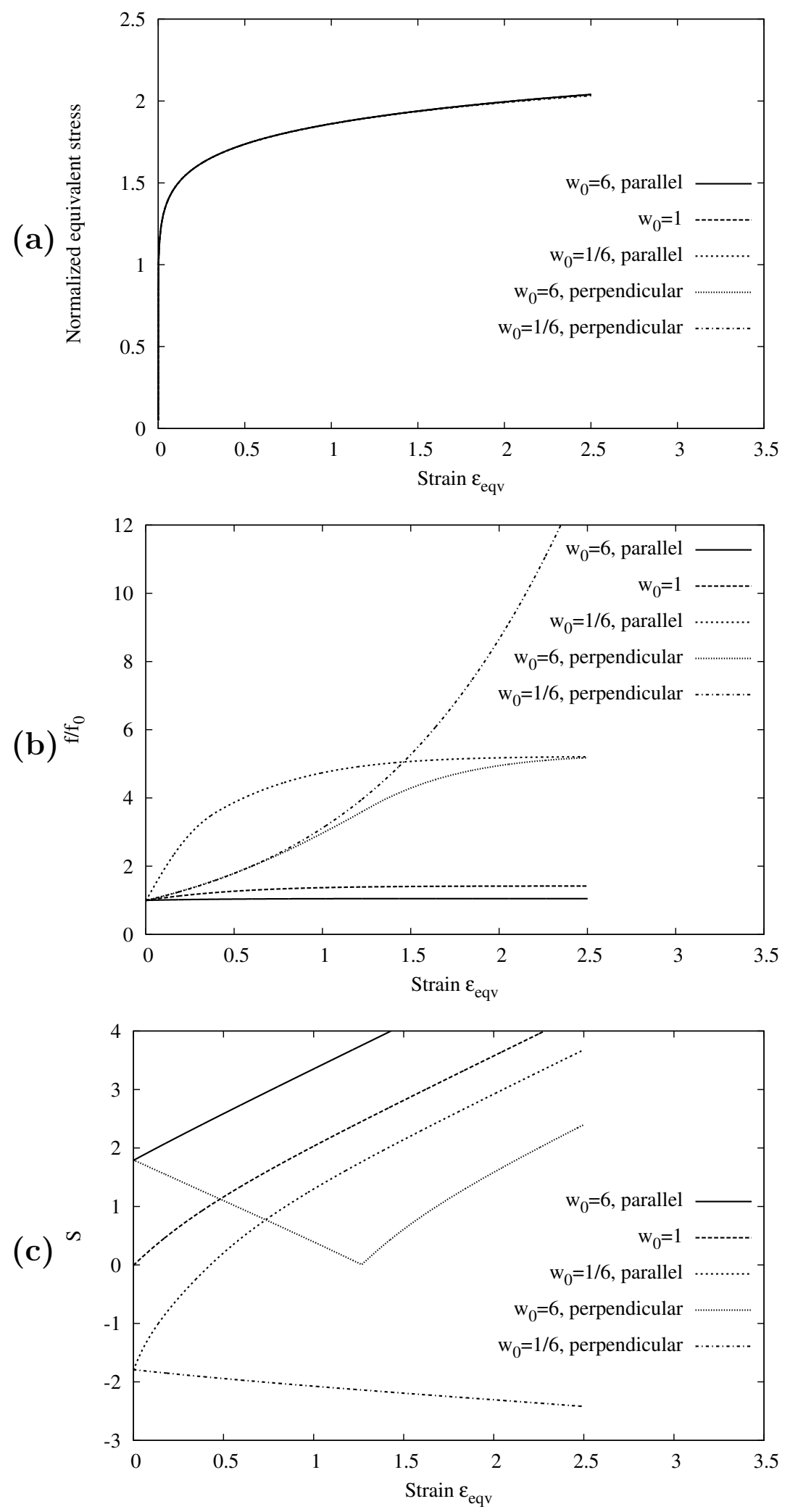

Figure 7: Parallel and perpendicular loadings to the void main axis for $\mathcal{T}=1 / 3$ and $f_{0}=$ 0.0001. Spheroidal void in the isotropic matrix. (a) Normalized stress, (b) normalized porosity $f / f_{0}$, (c) void aspect ratio $S=\ln w$. 

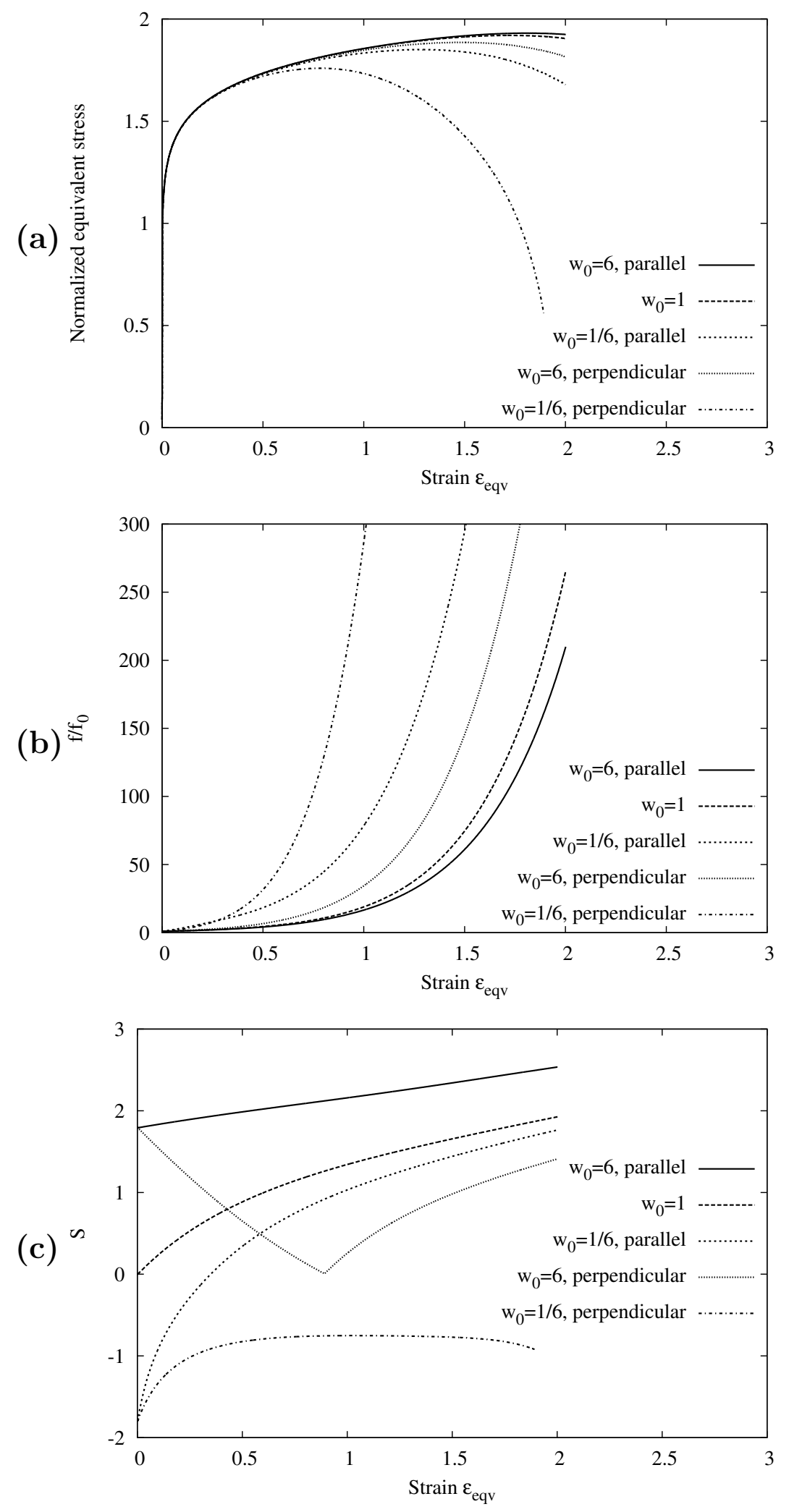

Figure 8: Parallel and perpendicular loadings to the void main axis for $\mathcal{T}=1$ and $f_{0}=$ 0.0001. Spheroidal void in the isotropic matrix. (a) Normalized stress, (b) normalized porosity $f / f_{0}$, (c) void aspect ratio $S=\ln w$. 


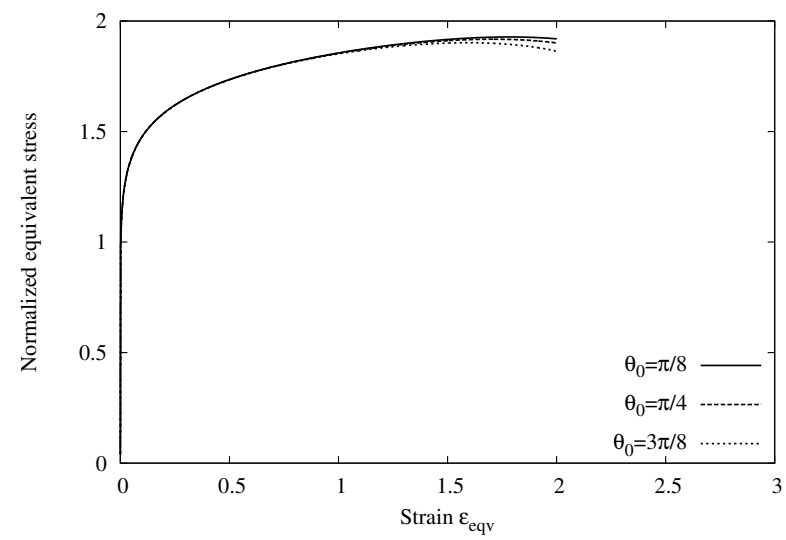

(a)

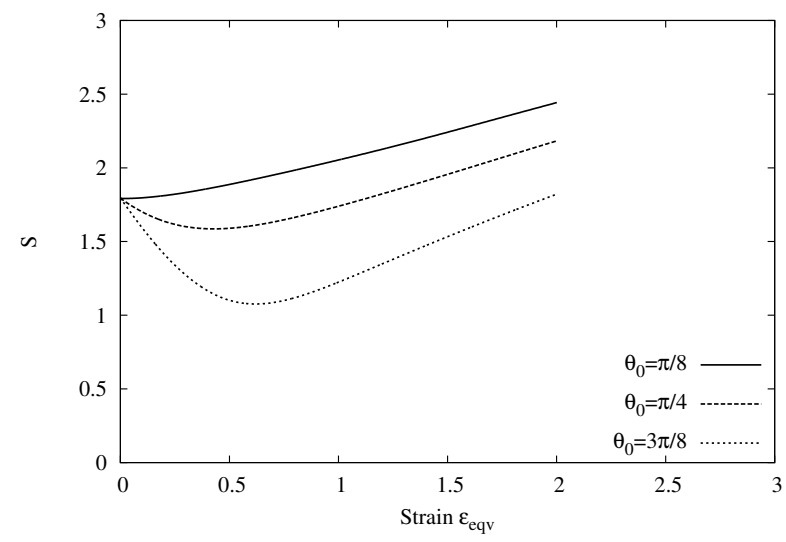

(c)

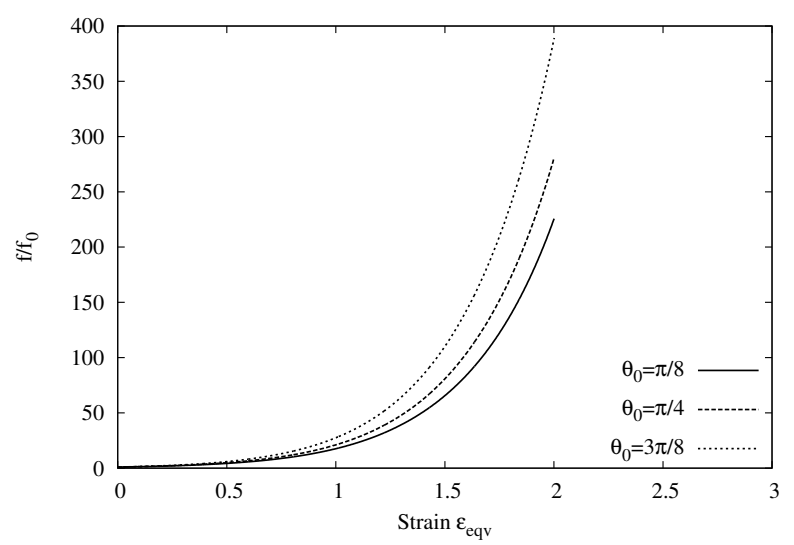

(b)

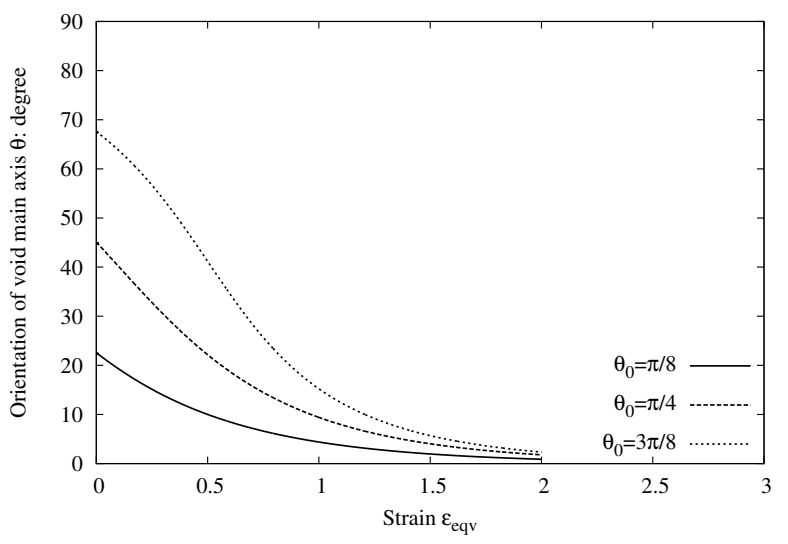

(d)

Figure 9: Misaligned loading for $\mathcal{T}=1, f_{0}=0.0001$ and $w_{0}=6$. Spheroidal void in the isotropic matrix. (a) Normalized stress, (b) normalized porosity $f / f_{0}$, (c) void aspect ratio $S=\ln w,(\mathrm{~d})$ orientation angle, $\theta$, of the void axis from the loading direction. 


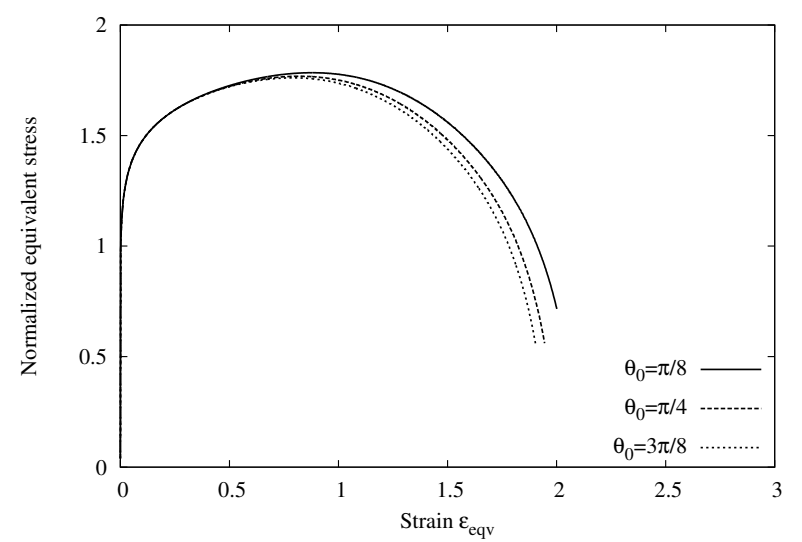

(a)

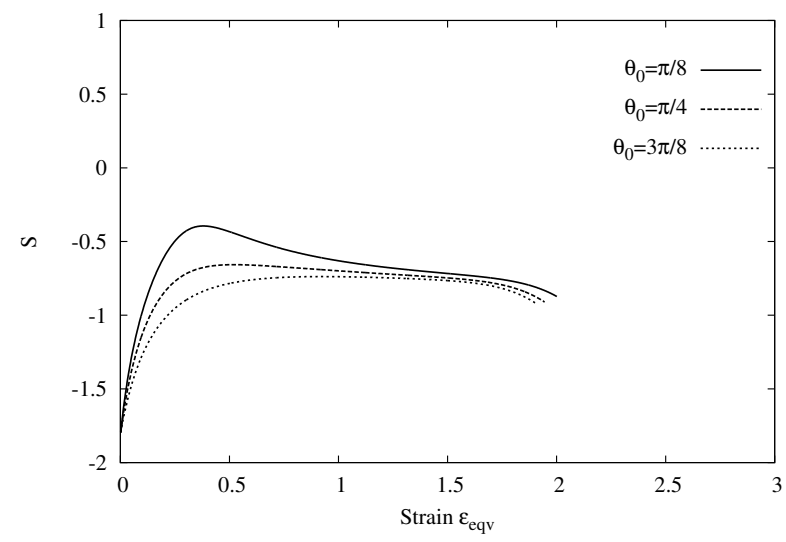

(c)

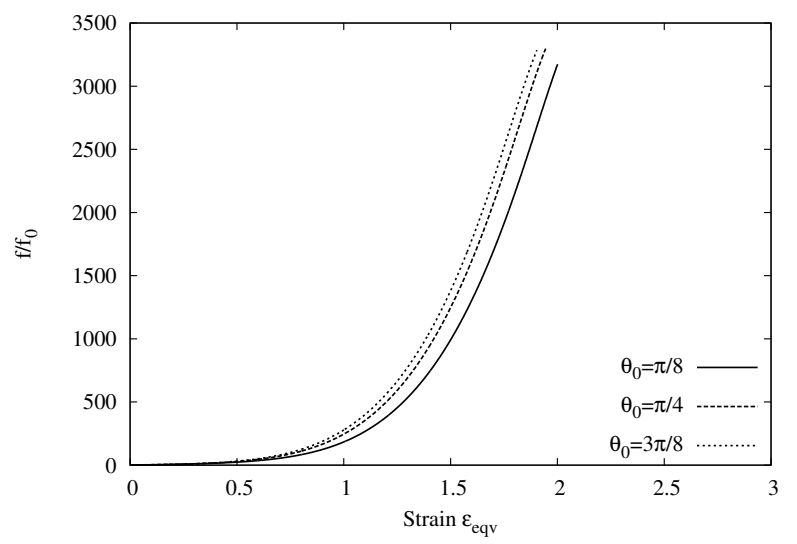

(b)

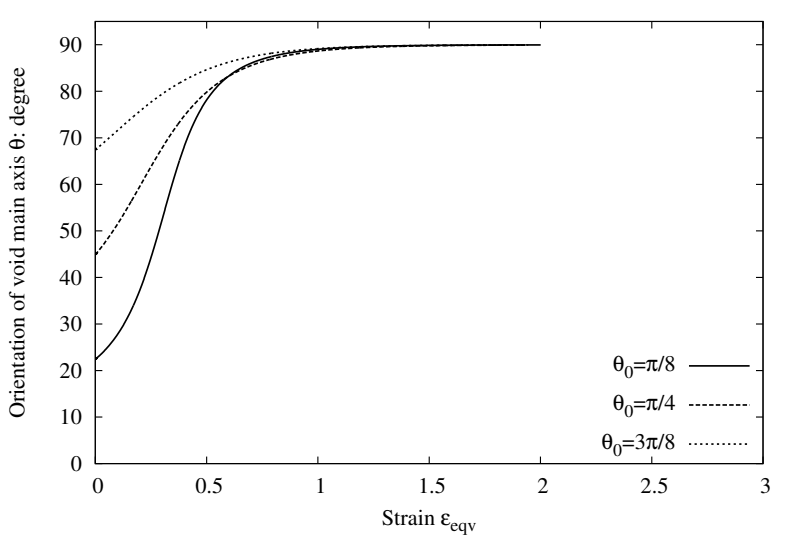

(d)

Figure 10: Misaligned loading for $\mathcal{T}=1, f_{0}=0.0001$ and $w_{0}=1 / 6$. Spheroidal void in the isotropic matrix. (a) Normalized stress, (b) normalized porosity $f / f_{0}$, (c) void aspect ratio $S=\ln w,(\mathrm{~d})$ orientation angle, $\theta$, of the void axis from the loading direction. 
(a)
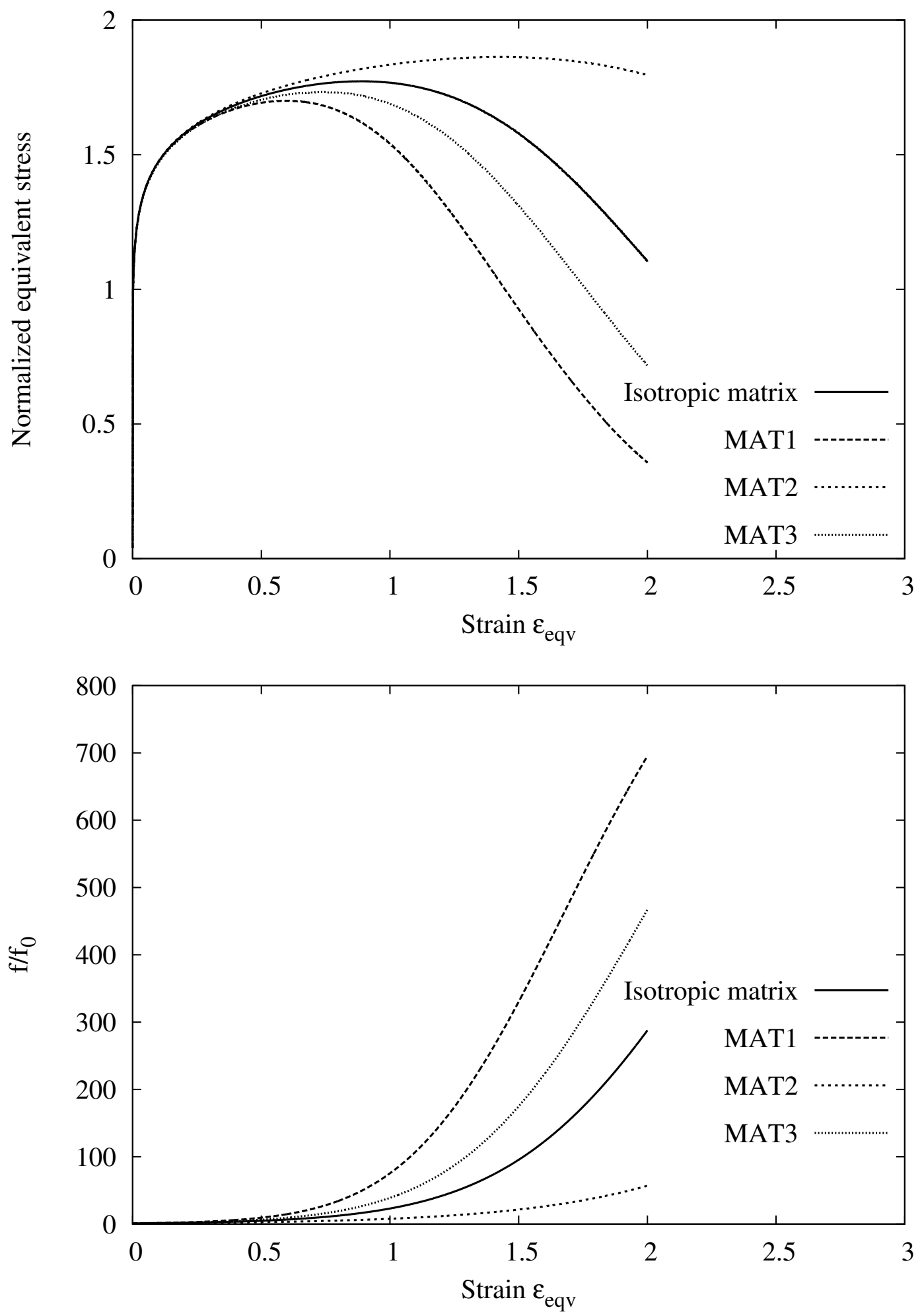

Figure 11: Spherical void in anisotropic matrices for $\mathcal{T}=1$ and $f_{0}=0.001$. (a) Normalized stress, (b) normalized porosity $f / f_{0}$. 

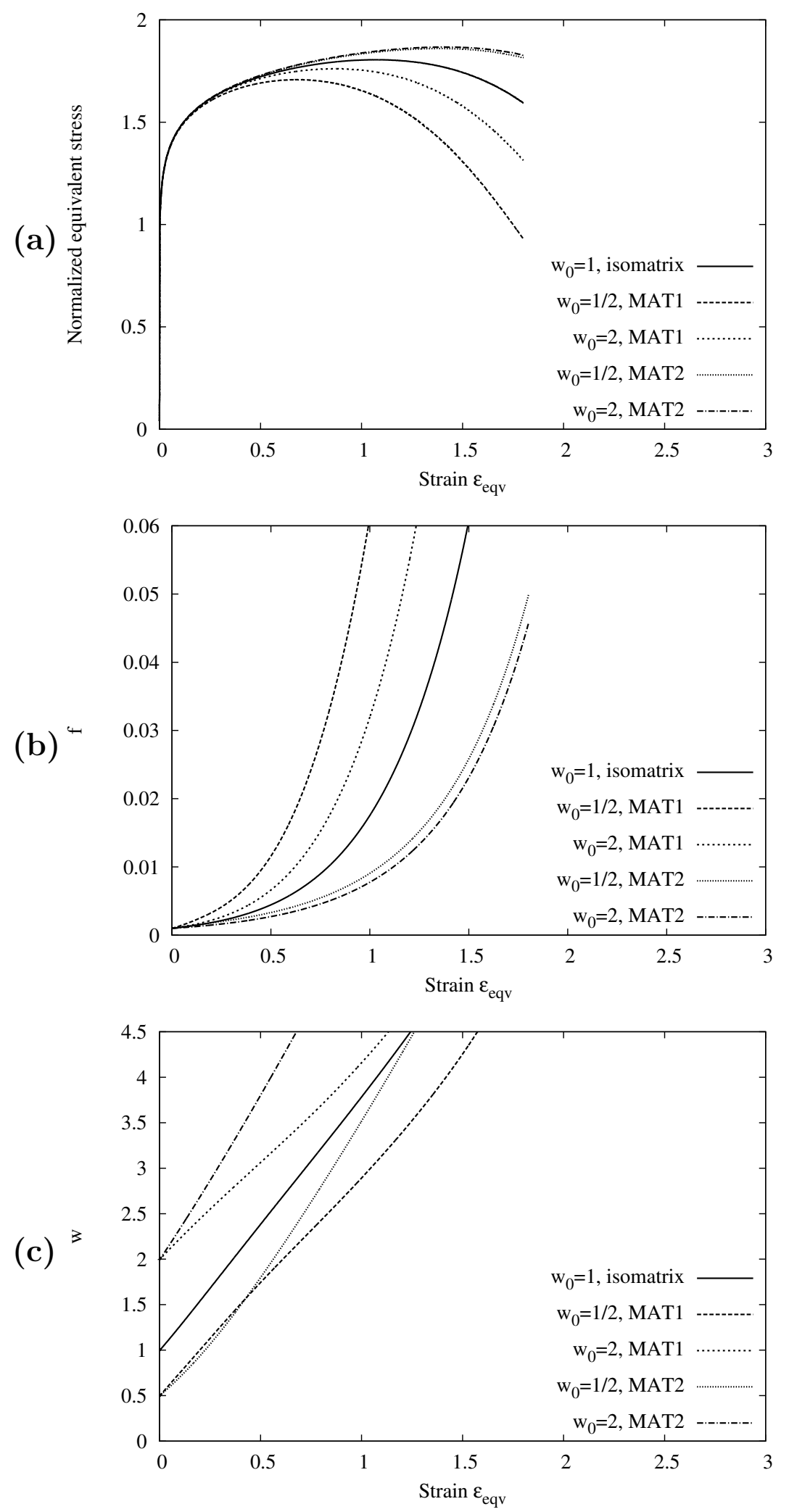

Figure 12: Parallel loading to the void main axis for $\mathcal{T}=1$ and $f_{0}=0.001$. Spheroidal void in the transversely isotropic matrices MAT1 and MAT2. (a) Normalized stress, (b) porosity $f,(\mathrm{c})$ void aspect ratio $w$. 

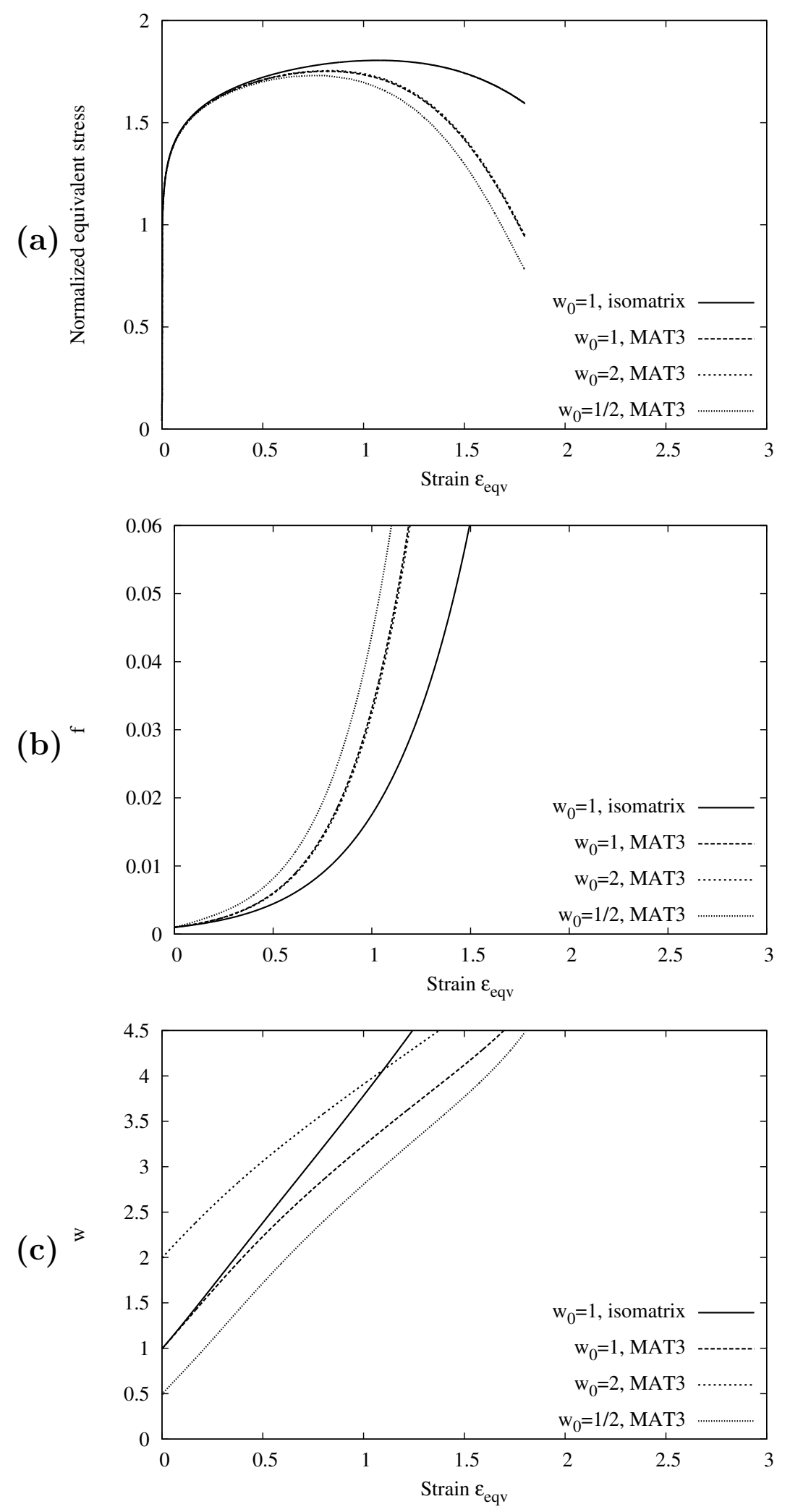

Figure 13: Parallel loading to the void main axis for $\mathcal{T}=1$ and $f_{0}=0.001$. Spheroidal void in the orthotropic matrix MAT3. (a) Normalized stress, (b) porosity $f$, (c) void aspect ratio $w$. 


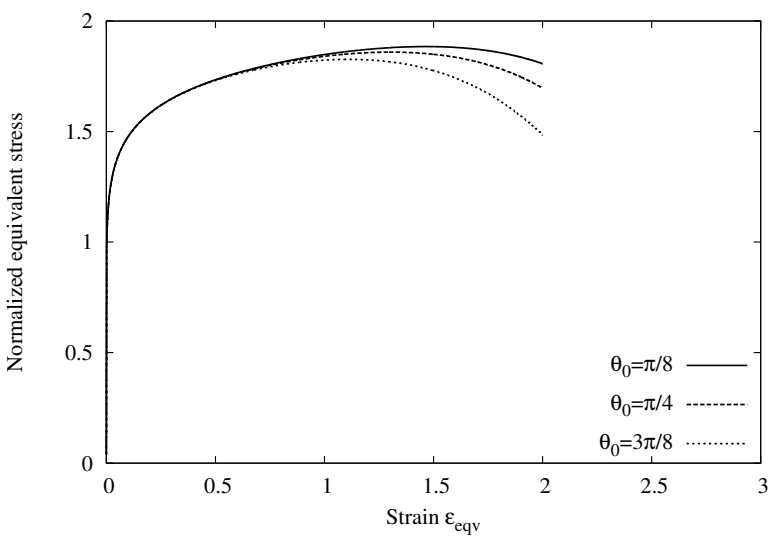

(a)

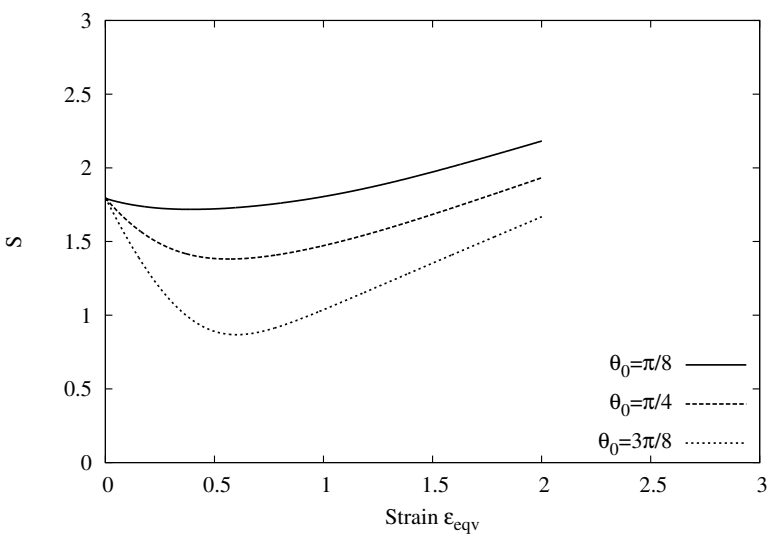

(c)

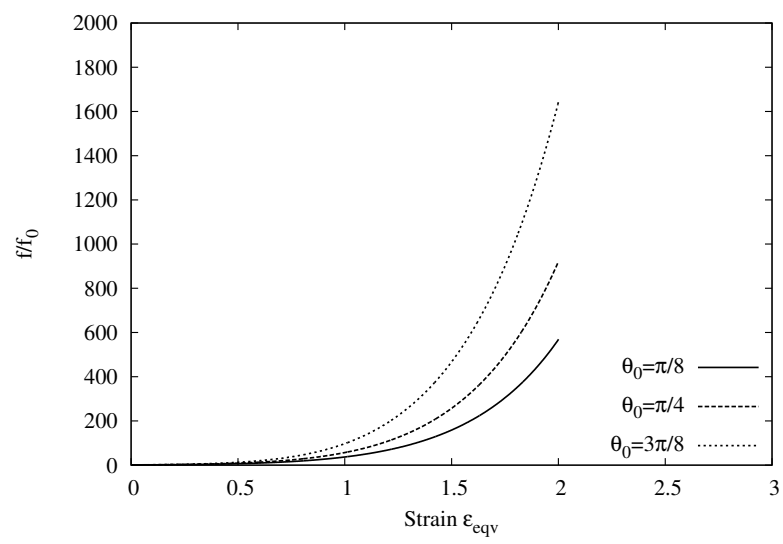

(b)

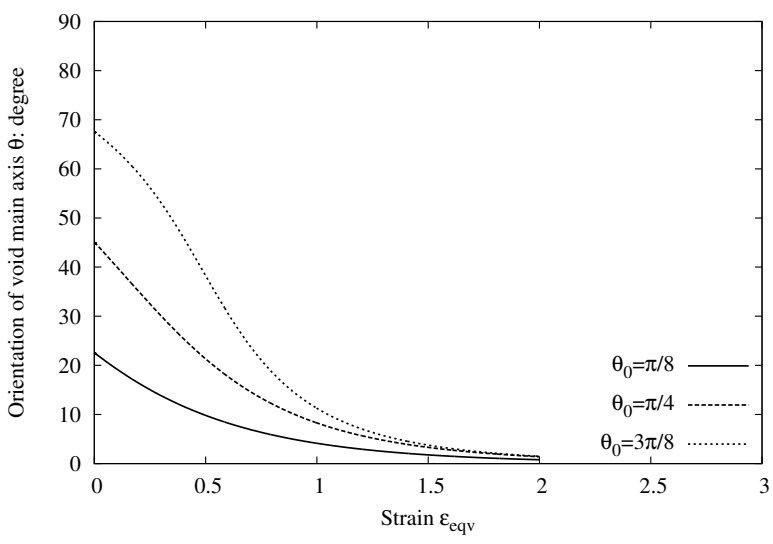

(d)

Figure 14: Misaligned loading for $\mathcal{T}=1, f_{0}=0.0001$ and $w_{0}=6$. Spheroidal void in matrix MAT1. (a) Normalized stress, (b) normalized porosity $f / f_{0}$, (c) void aspect ratio $S=\ln w$, (d) orientation angle, $\theta$, of the void axis from the loading direction. 


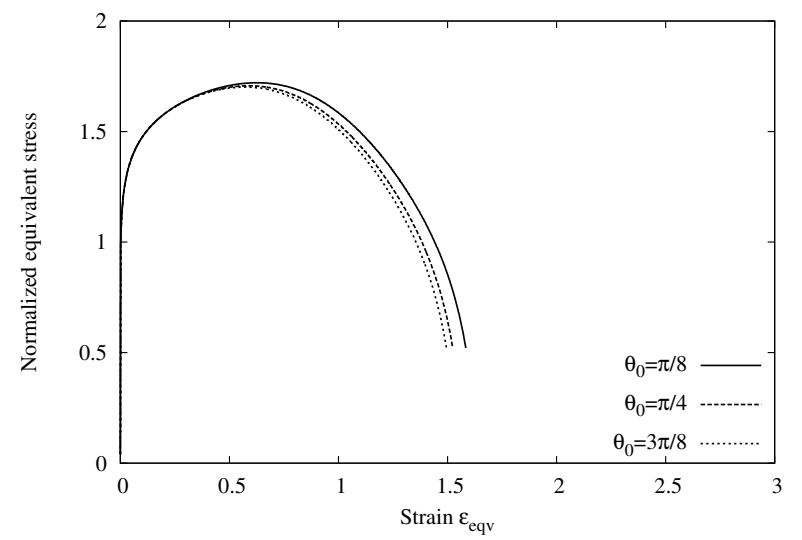

(a)

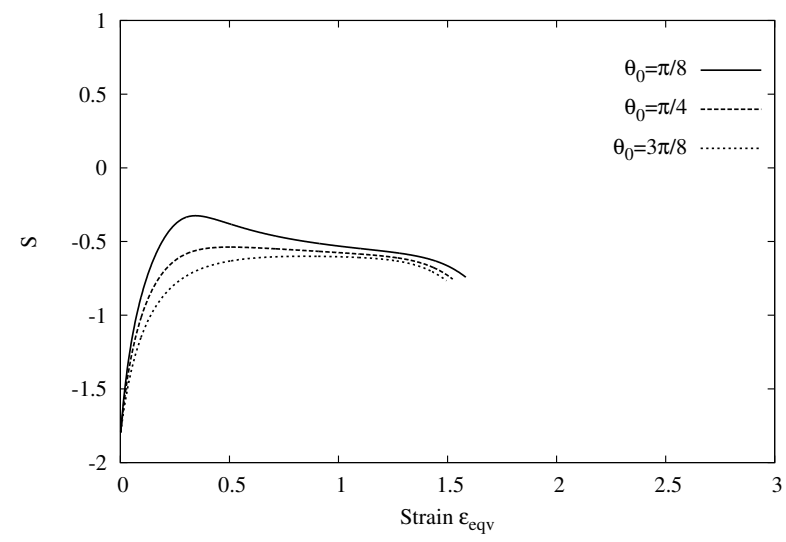

(c)

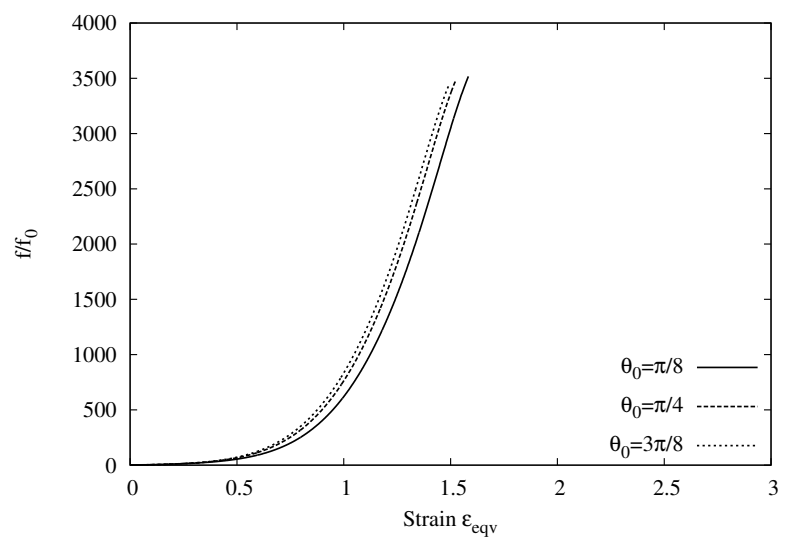

(b)

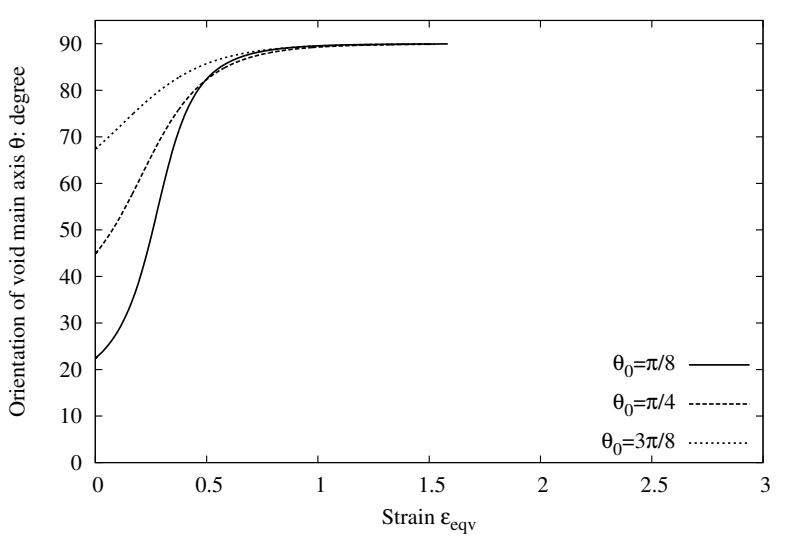

(d)

Figure 15: Misaligned loading for $\mathcal{T}=1, f_{0}=0.0001$ and $w_{0}=1 / 6$. Spheroidal void in matrix MAT1. (a) Normalized stress, (b) normalized porosity $f / f_{0}$, (c) void aspect ratio $S=\ln w$, (d) orientation angle, $\theta$, of the void axis from the loading direction. 


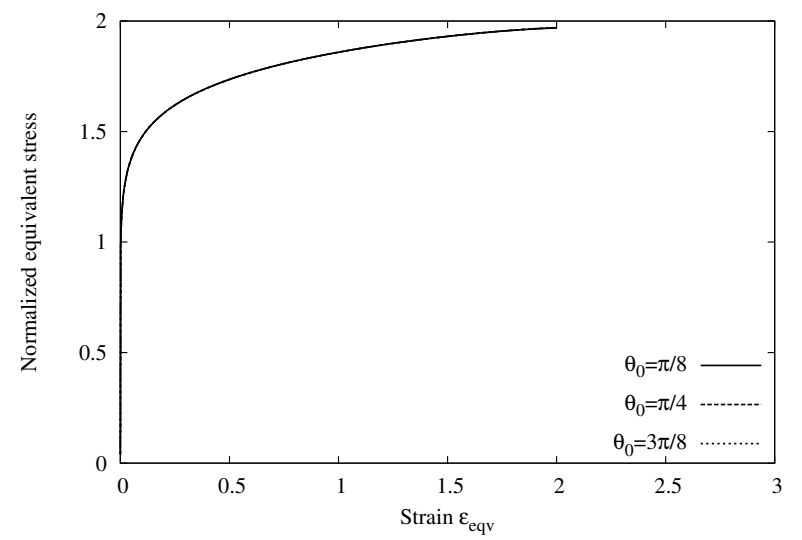

(a)

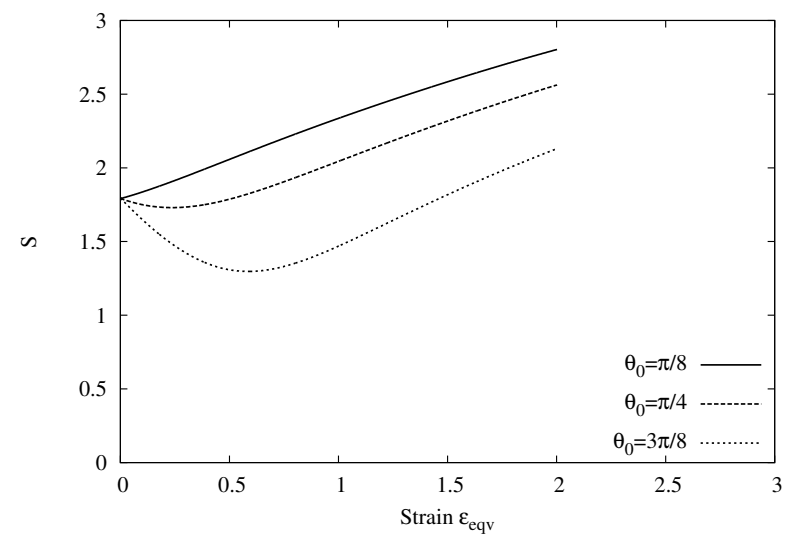

(c)

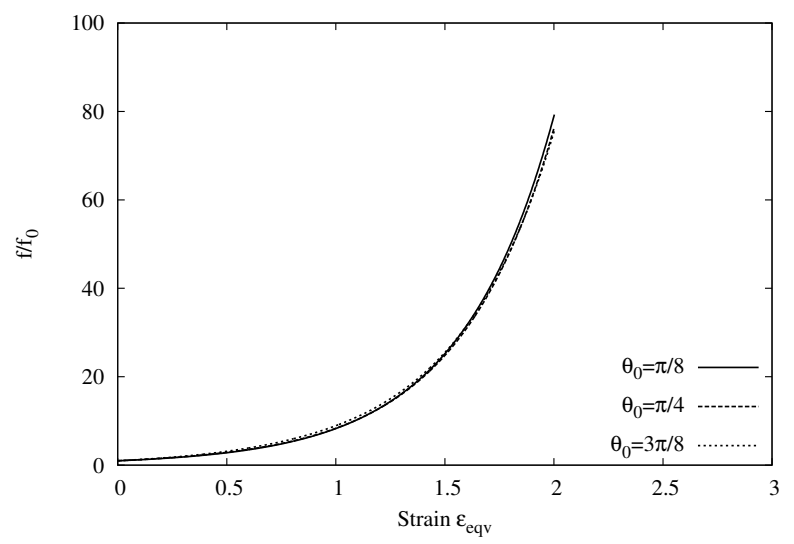

(b)

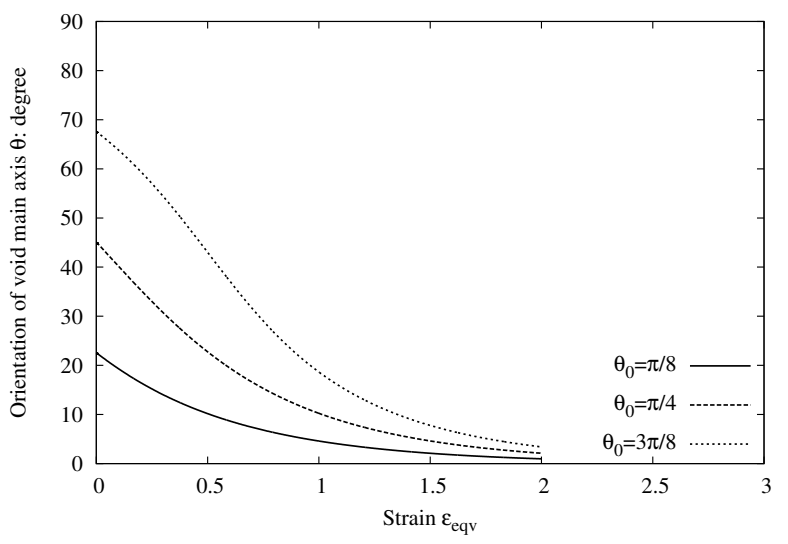

(d)

Figure 16: Misaligned loading for $\mathcal{T}=1, f_{0}=0.0001$ and $w_{0}=6$. Spheroidal void in matrix MAT2. (a) Normalized stress, (b) normalized porosity $f / f_{0}$, (c) void aspect ratio $S=\ln w$, (d) orientation angle, $\theta$, of the void axis from the loading direction. 


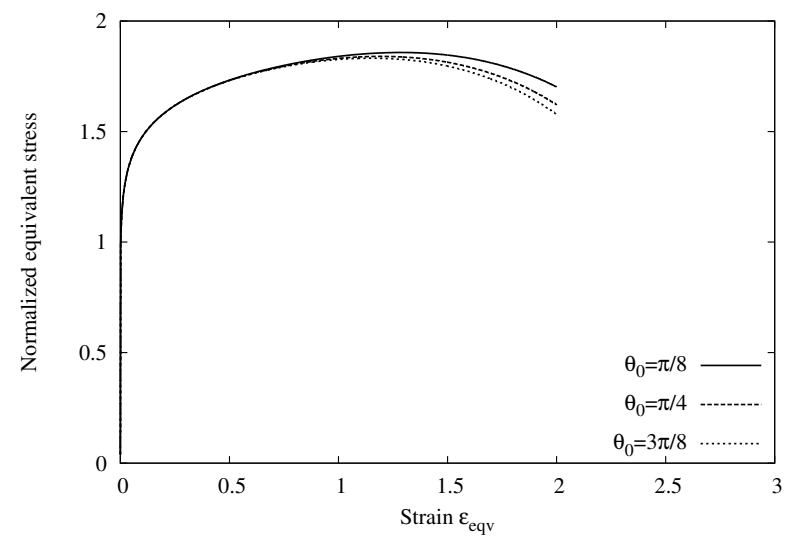

(a)

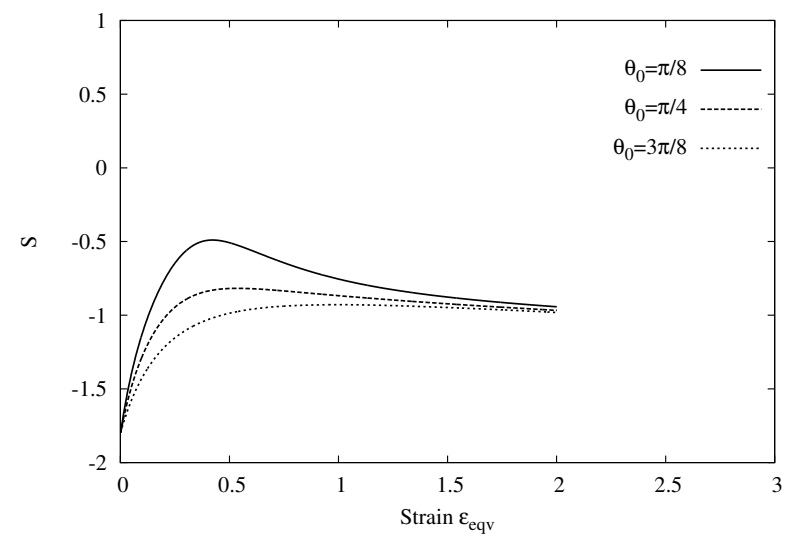

(c)

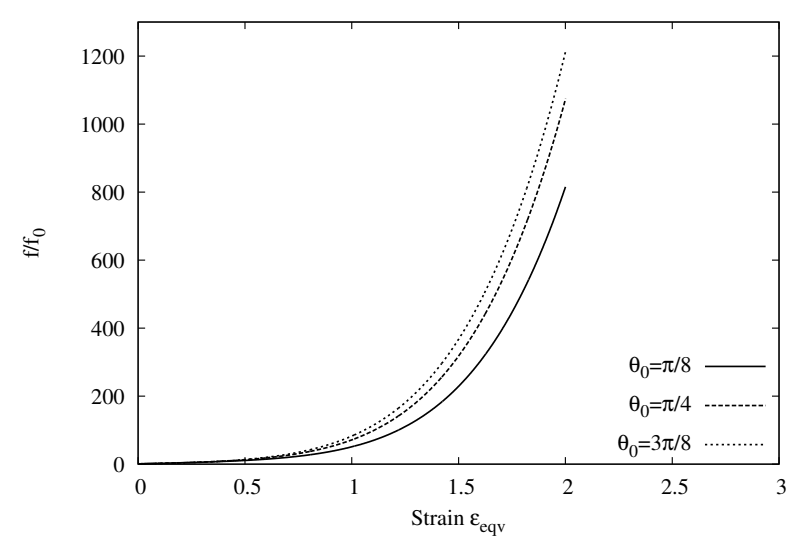

(b)

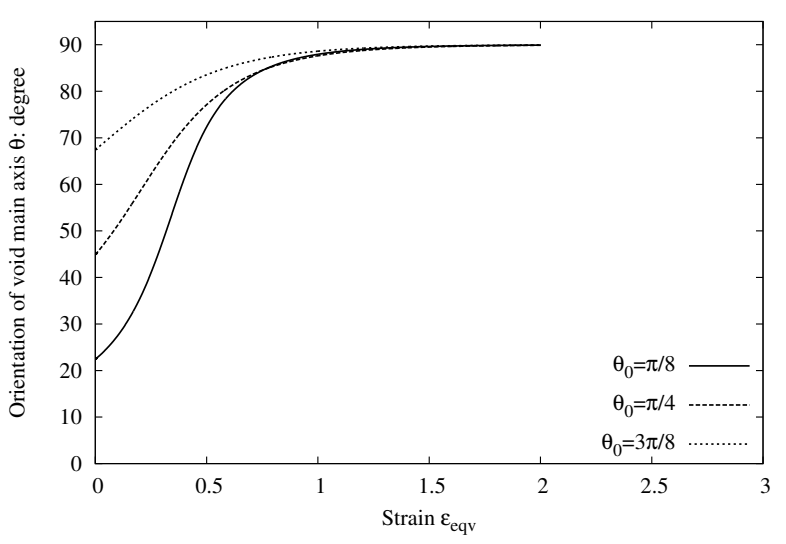

(d)

Figure 17: Misaligned loading for $\mathcal{T}=1, f_{0}=0.0001$ and $w_{0}=1 / 6$. Spheroidal void in matrix MAT2. (a) Normalized stress, (b) normalized porosity $f / f_{0}$, (c) void aspect ratio $S=\ln w$, (d) orientation angle, $\theta$, of the void axis from the loading direction. 


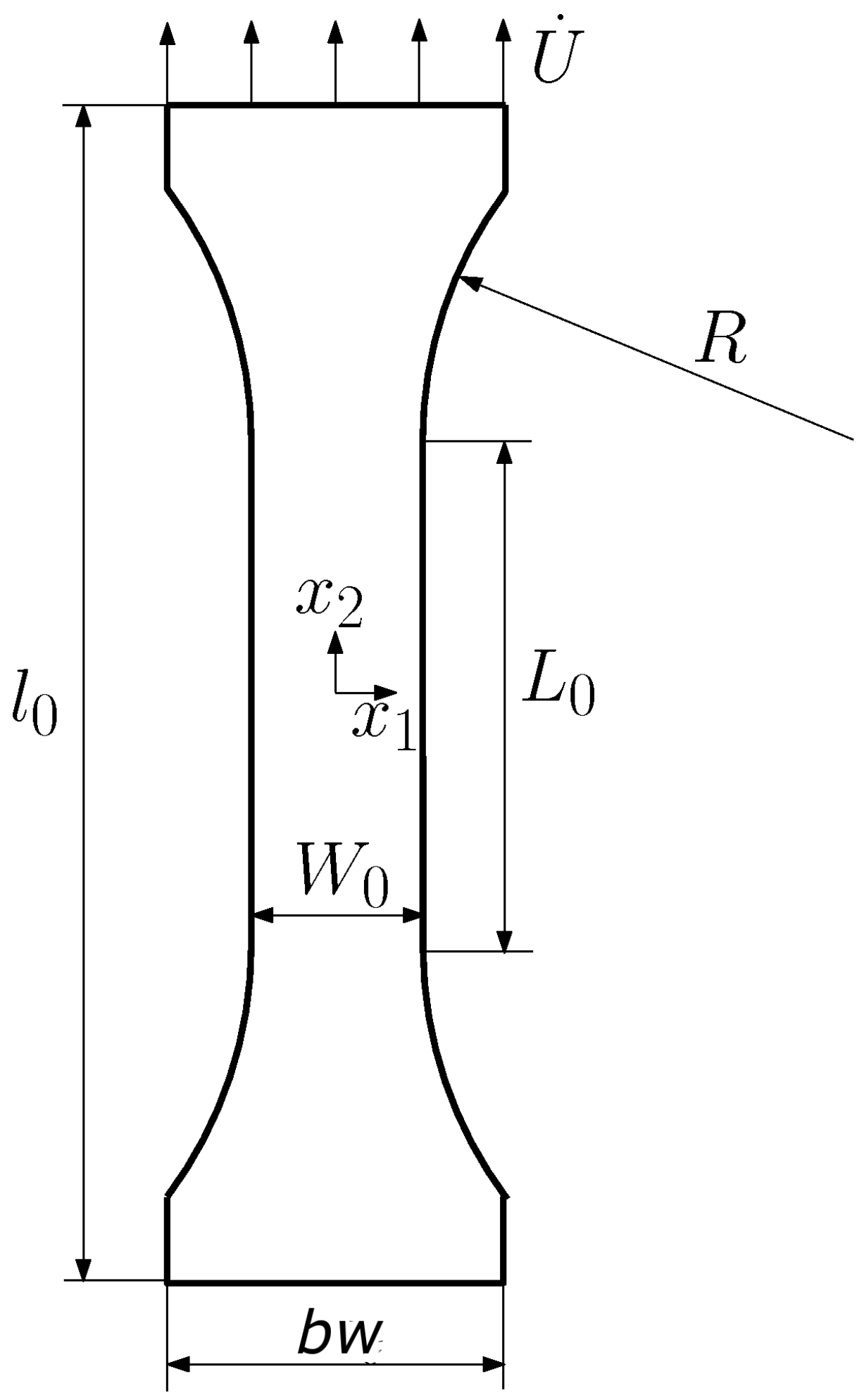

Figure 18: Geometry of tensile specimen and other definitions. 

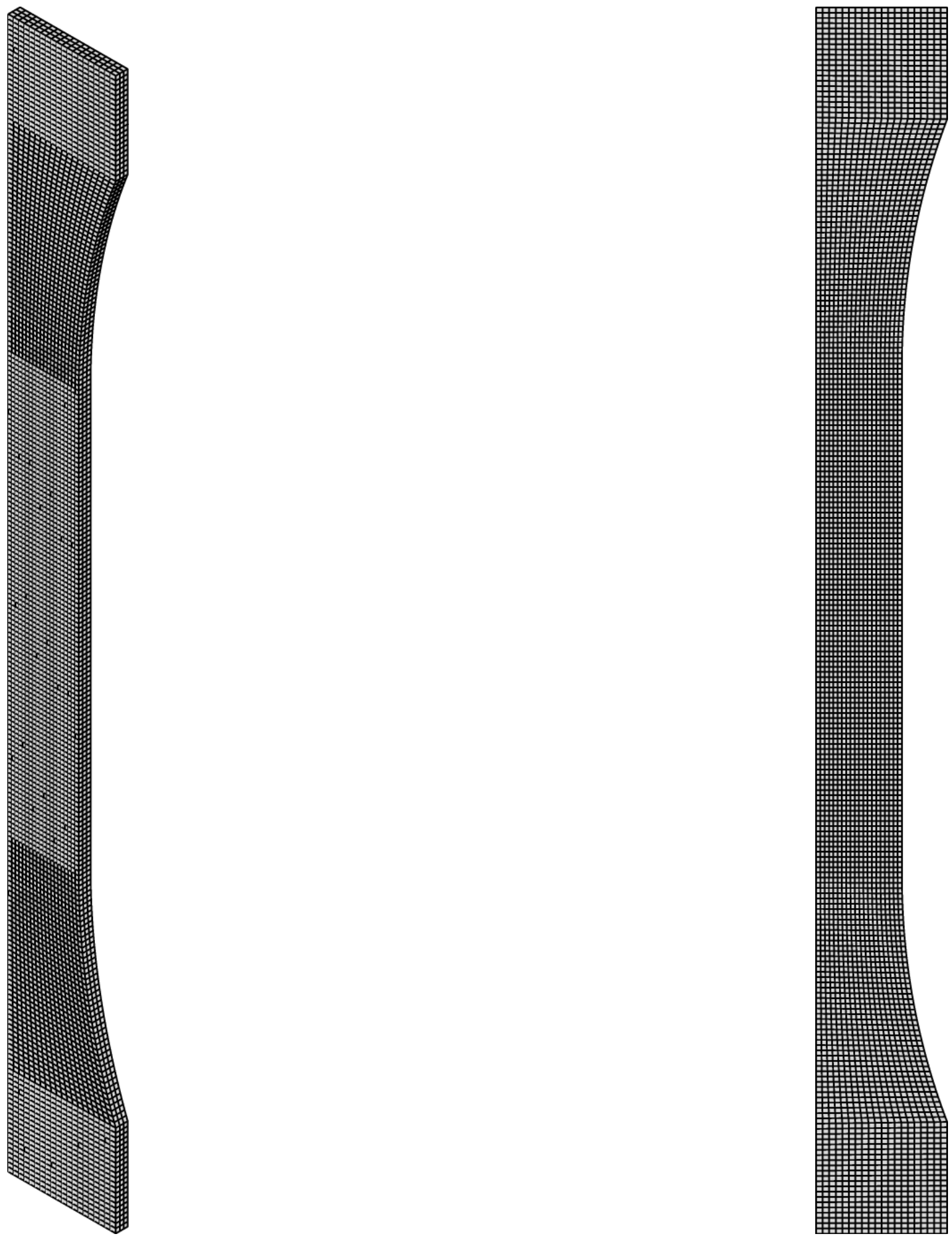

(a)

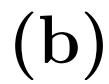

Figure 19: Finite element meshes used for (a) the 3D tensile specimen and (b) the 2D tensile specimen, i.e., axi-symmetric and plane strain. 
(a)

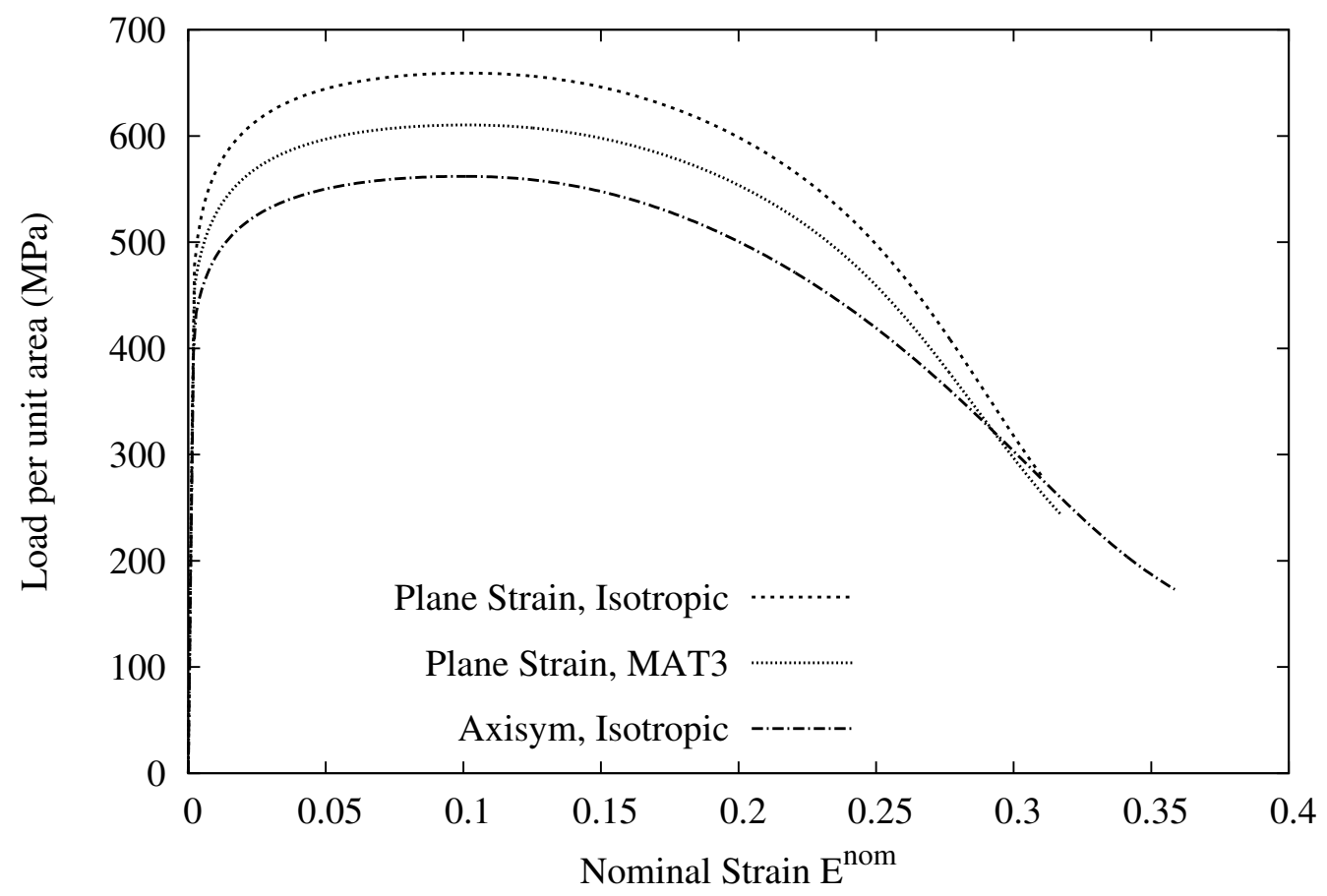

(b)

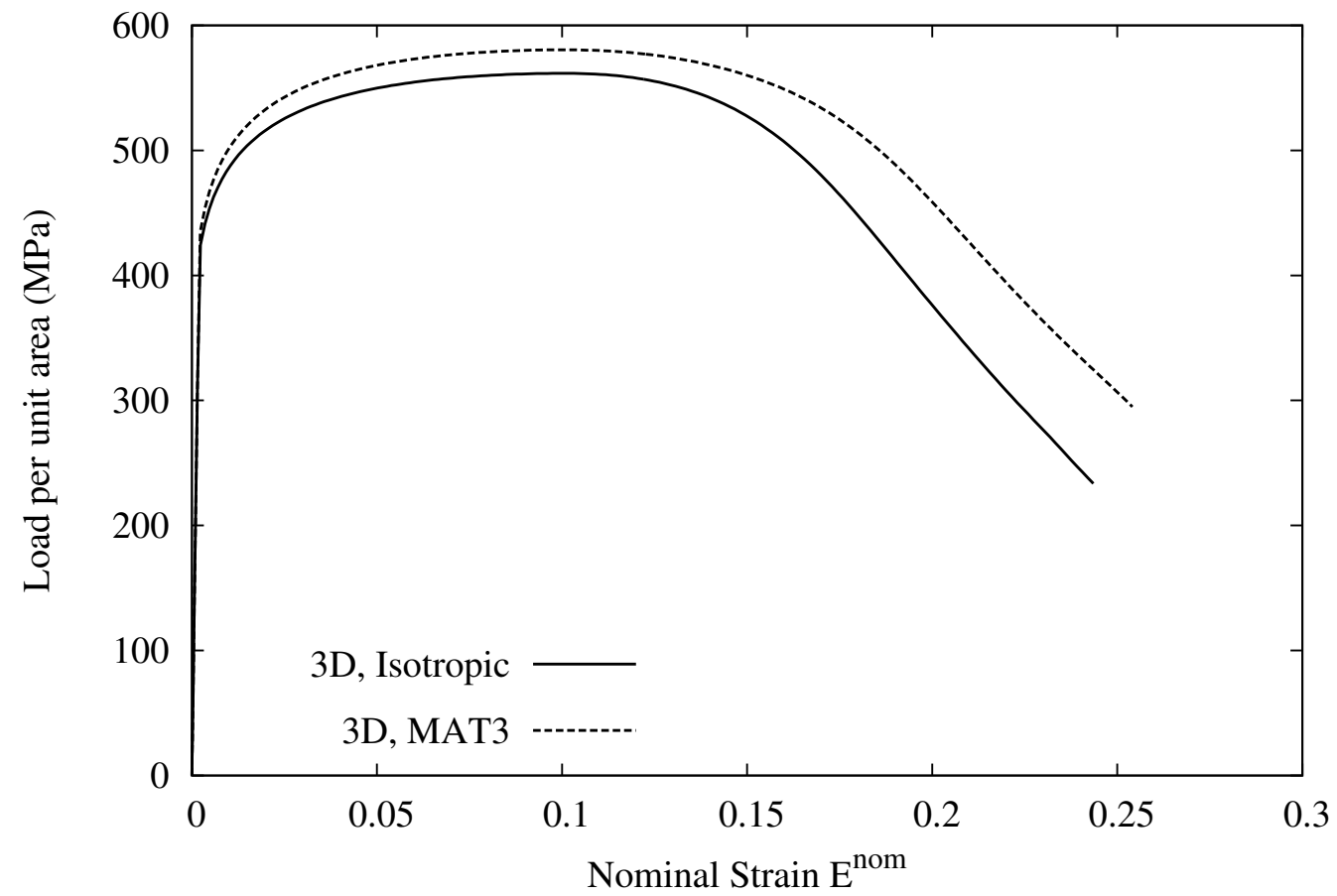

Figure 20: Load normalized by the initial area versus nominal strain for (a) the 2D and (b) 3D calculations. 


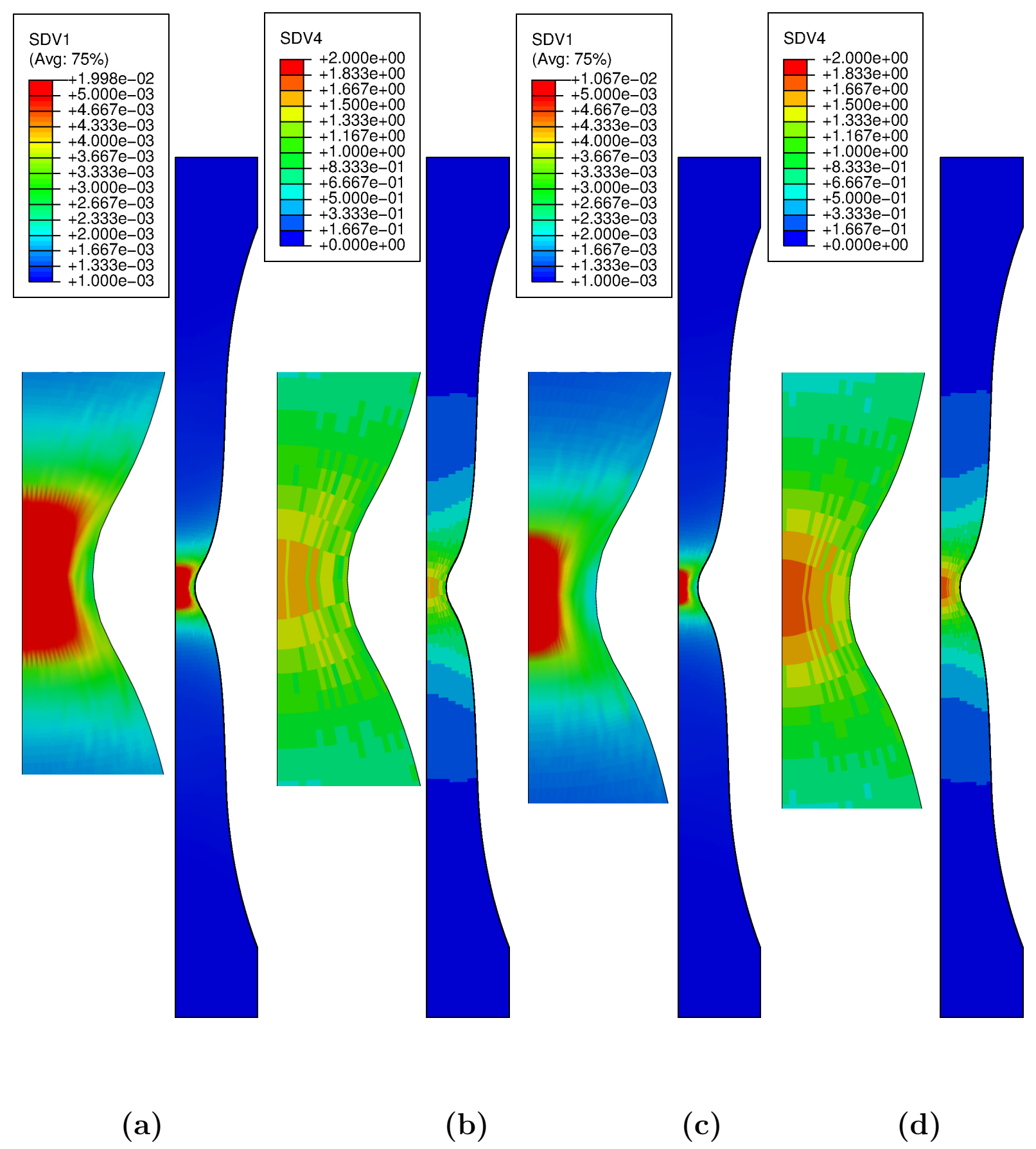

Figure 21: Contours of porosity $f$ (interpolated at nodes) and void aspect ratio $S=\ln w$ (element averaged) for the plane strain tensile specimen simulations at $E=1.0$. (a) $f$, isotropic matrix; (b) $S$, isotropic matrix; (c) $f$, MAT3, (d) $S$, MAT3. Insets show enlarged views of necks. 


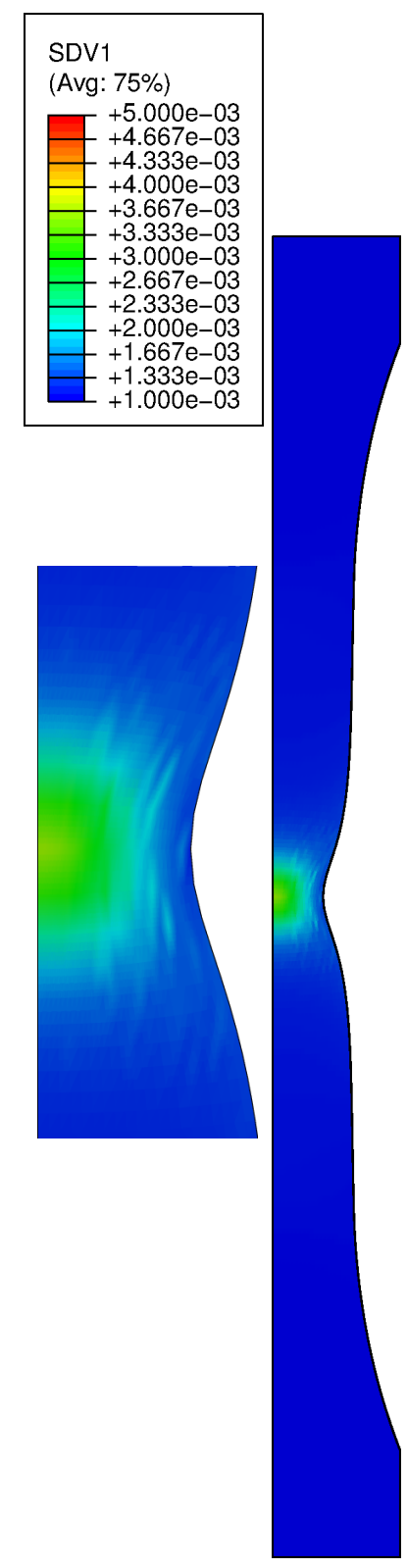

(a)

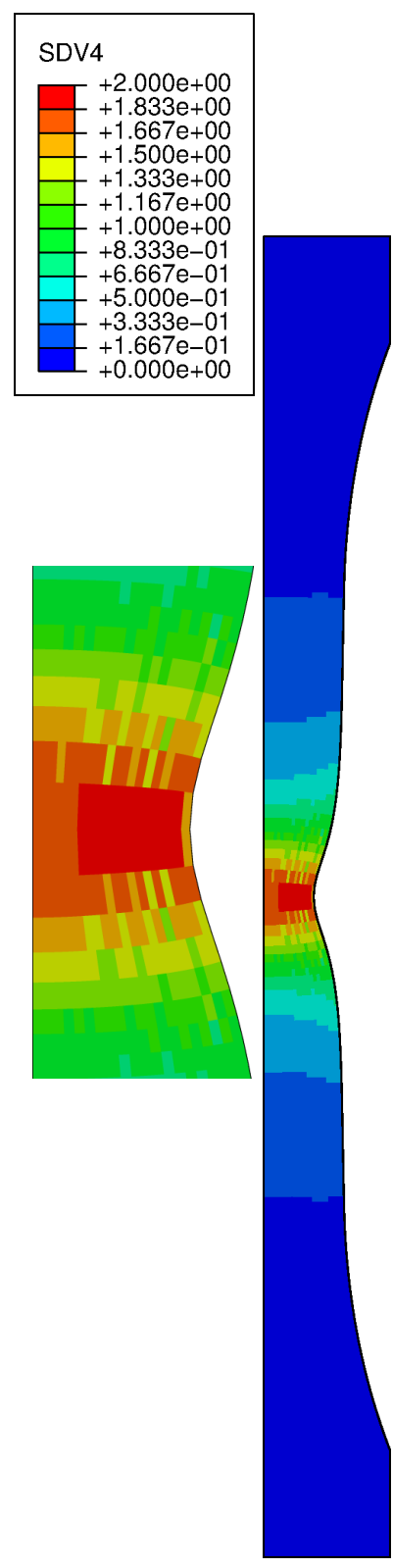

(b)

Figure 22: Contours of porosity $f$ and void aspect ratio $S=\ln w$ for the axisymmetric tensile specimen simulation at nominal strain $E=1.0$. Only the isotropic matrix is used. (a) $f$ (nodal), (b) $S=\ln w$ (element averaged). 


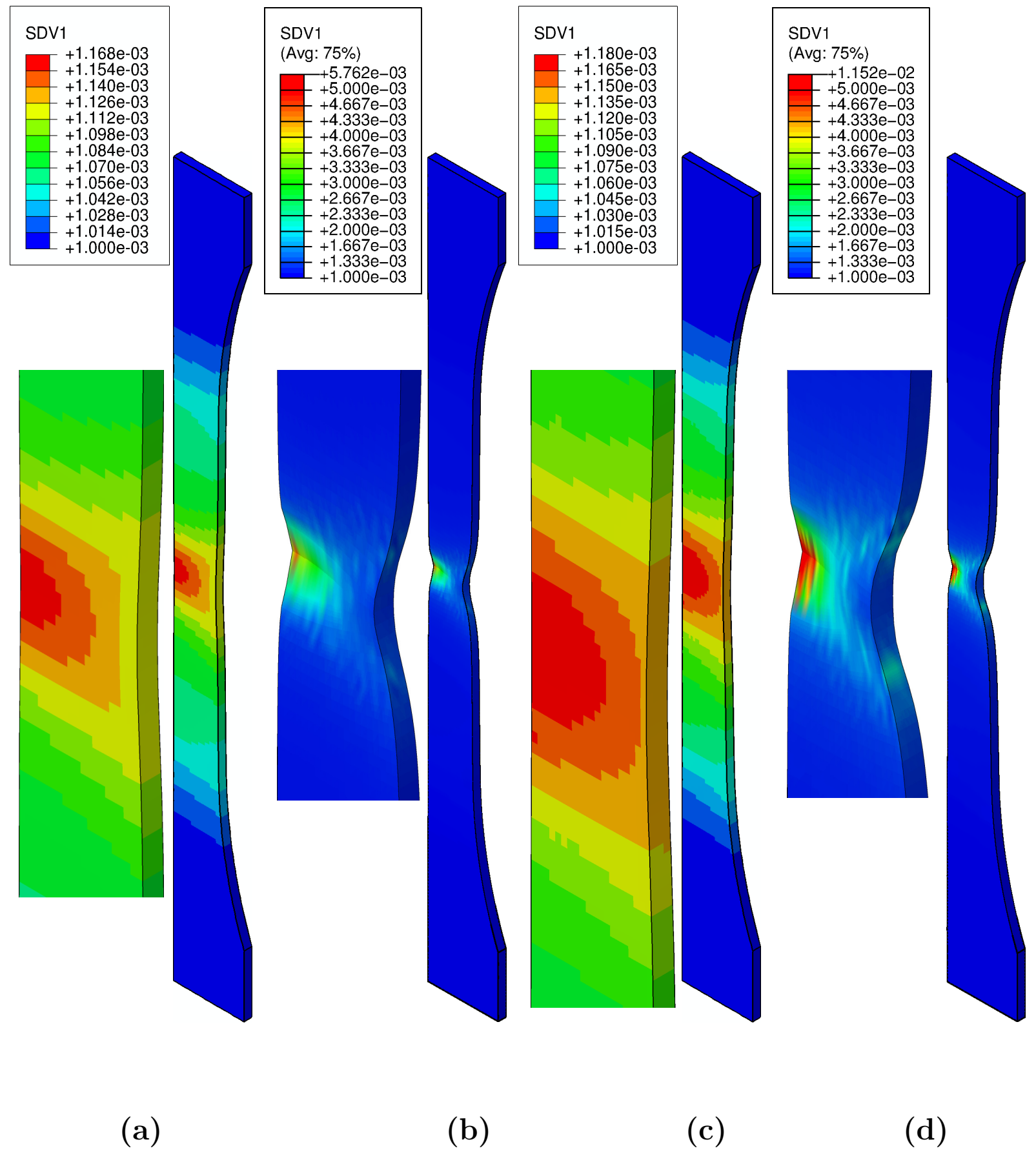

Figure 23: Contours of porosity $f$ for the 3D tensile specimen (a),(c) at nominal strain $E^{\text {nom }}=0.15$ (element averaged) and (b),(d) at $E=1.0$ (interpolated at nodes). (a),(b) Isotropic matrix, (c),(d) MAT3. 

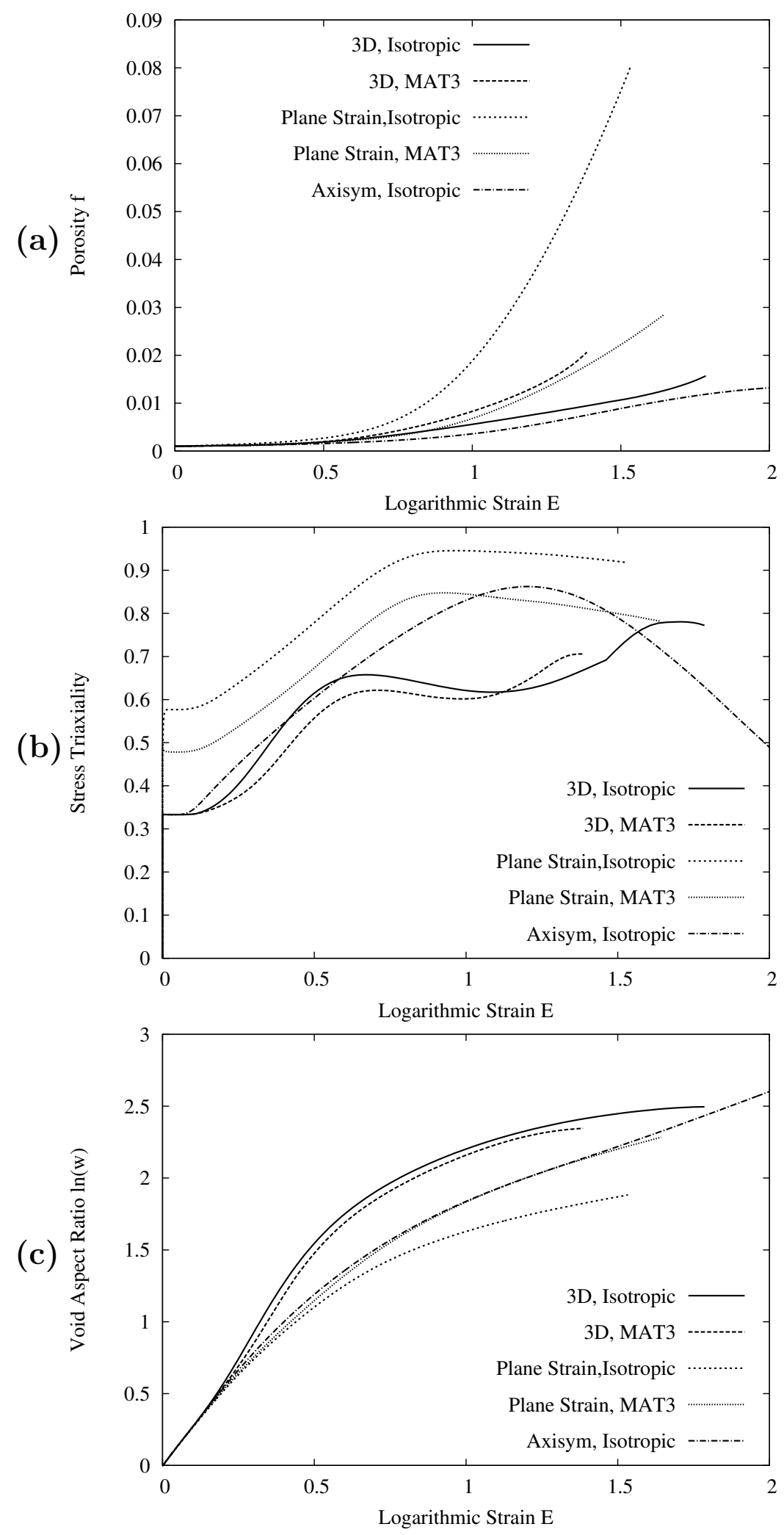

Figure 24: Evolution with the logarithmic strain $E$ of (a) porosity $f$, (b) stress triaxiality $\mathcal{T}$, (c) void aspect ratio $S=\ln w$ at the center of the specimens. The line type is the same as in Fig. 20. 


\section{References}

[1] A. L. Gurson. Continuum theory of ductile rupture by void nucleation and growth: Part iyield criteria and flow rules for porous ductile media. J. Engrg. Mat. Tech., 99:2-15, 1977.

[2] V. Tvergaard and A. Needleman. Analysis of the cup-cone fracture in a round tensile bar. Acta metall., 32:157-169, 1984.

[3] A. A. Benzerga and J.-B. Leblond. Ductile fracture by void growth to coalescence. Adv. Appl. Mech., 44:169-305, 2010.

[4] G. Rousselier. Ductile fracture models and their potential in local approach of fracture. Nucl. Eng. Design, 105:97-111, 1987.

[5] J. Lemaitre. A Continuous Damage Mechanics Model for Ductile Fracture. J. Engrg. Mat. Tech., 107:83-89, 1985.

[6] R. K. Abu Al-Rub and G. Z. Voyiadjis. On the coupling of anisotropic damage and plasticity models for ductile materials. Int. J. Solids Struct., 40(11):2611 - 2643, 2003.

[7] J. Besson. Continuum Models of Ductile Fracture: A Review. Int. J. Damage Mech., 19:3-52, 2010.

[8] M. Garajeu, J. C. Michel, and P. Suquet. A micromechanical approach of damage in viscoplastic materials by evolution in size, shape and distribution of voids. Comput. Methods Appl. Mech. Engrg., 183:223-246, 2000.

[9] S. Hao, W. K. Liu, and C. T. Chang. Computer implementation of damage models by finite element and mesh free methods. Comput. Methods Appl. Mech. Engrg., 187:401$440,2000$.

[10] K. Danas and P. Ponte Castañeda. A finite-strain model for anisotropic viscoplastic porous media: I-Theory. Eur. J. Mech., 28:387-401, 2009.

[11] S. Ghosh, J. Bai, and D. Paquet. Homogenization-based continuum plasticity-damage model for ductile failure of materials containing heterogeneities. J. Mech. Phys. Solids, 57:1017-1044, 2009.

[12] S. Ganapathysubramanian and N. Zabaras. Computational design of deformation processes for materials with ductile damage. Comput. Methods Appl. Mech. Engrg., 192:147-183, 2003.

[13] K. Danas and N. Aravas. Numerical modeling of elasto-plastic porous materials with void shape effects at finite deformations. Composites Part B: Engineering, 43:2544-2559, 2012.

[14] W. Rajhi, K. Saanouni, and H. Sidhom. Anisotropic ductile damage fully coupled with anisotropic plastic flow: Modeling, experimental validation, and application to metal forming simulation. Int. J. Damage Mech., 23:1211-1256, 2014. 
[15] J.-B. Leblond and G. Perrin. Bifurcation effects in ductile metals with damage delocalization. J. Appl. Mech., 61:236-242, 1994.

[16] V. Tvergaard and A. Needleman. Effects of nonlocal damage in porous plastic solids. Int. J. Solids Struct., 32:1063-1077, 1995.

[17] K. Enakoutsa, J.-B. Leblond, and G. Perrin. Numerical implementation and assessment of a phenomenological nonlocal model of ductile rupture. Comput. Methods Appl. Mech. Engrg., 196:1946-1957, 2007.

[18] H. Baaser and V. Tvergaard. A new algorithmic approach treating nonlocal effects at finite rate-independent deformation using the Rousselier damage model. Comput. Methods Appl. Mech. Engrg., 192:107-124, 2003.

[19] J. Mediavilla, R. H. J. Peerlings, and M. G. D. Geers. A nonlocal triaxiality-dependent ductile damage model for finite strain plasticity. Comput. Methods Appl. Mech. Engrg., 195:4617-4634, 2006.

[20] J.-M. Bergheau, J.-B. Leblond, and P. Perrin. A new numerical implementation of a second-gradient model for plastic porous solids, with an application to the simulation of ductile rupture tests. Comput. Methods Appl. Mech. Engrg., 268:105-125, 2014.

[21] E. Lorentz, J. Besson, and V. Cano. Numerical simulation of ductile fracture with the rousselier constitutive law. Comput. Methods Appl. Mech. Engrg., 197:1965-1982, 2008.

[22] J. P. Crété, P. Longère, and J. M. Cadou. Numerical modelling of crack propagation in ductile materials combining the gtn model and x-fem. Comput. Methods Appl. Mech. Engrg., 275:204-233, 2014.

[23] A.E. Huespe, A. Needleman, J. Oliver, and P.J. Sanchez. A finite strain, finite band method for modeling ductile fracture. Int. J. Plasticity, 28:53-69, 2012.

[24] F. Barlat, H. Aretz, J. W. Yoon, M. E. Karabin, J. C. Brem, and R. E. Dick. Linear transfomation-based anisotropic yield functions. Int. J. Plasticity, 21(5):1009-1039, 2005.

[25] S. Oller, E. Car, and J. Lubliner. Definition of a general implicit orthotropic yield criterion. Comput. Methods Appl. Mech. Engrg., 192:895-912, 2003.

[26] F. Bron and J. Besson. A yield function for anisotropic materials Application to aluminum alloys. Int. J. Plasticity, 20:937-963, 2004.

[27] A. Pineau, A. A. Benzerga, and T. Pardoen. Failure of metals I. Brittle and Ductile Fracture. Acta Mater., 107:424-483, 2016.

[28] D. Mohr and S.J. Marcadet. Micromechanically-motivated phenomenological HosfordCoulomb model for predicting ductile fracture initiation at low stress triaxialities. Int. J. Solids Struct., 67-68:40-55, 2015. 
[29] J.W. Ju. Isotropic and anisotropic damage variables in continuum damage mechanics. Journal of Engineering Mechanics, 116:2764-2770, 1990.

[30] A. Menzel, M. Ekh, P. Steinmann, and K. Runesson. Anisotropic damage coupled to plasticity: Modelling based on the effective configuration concept. Int. J. Numer. Meths. Engrg., 54:1409-1430, 2002.

[31] M. Brünig. Numerical analysis of anisotropic ductile continuum damage. Comput. Methods Appl. Mech. Engrg., 192:2949-2976, 2003.

[32] M. Gologanu, J.-B. Leblond, and J. Devaux. Approximate models for ductile metals containing non-spherical voids - case of axisymmetric prolate ellipsoidal cavities. $J$. Mech. Phys. Solids, 41(11):1723-1754, 1993.

[33] P. Ponte Castañeda and M. Zaidman. Constitutive models for porous materials with evolving microstructure. J. Mech. Phys. Solids, 42:1459-1495, 1994.

[34] M. Gologanu, J-B. Leblond, G. Perrin, and J. Devaux. Recent extensions of gurson's model for porous ductile metals. pages 61-130, 1997. in: P. Suquet (Ed.), Continuum micromechanics, CISM Courses and Lectures, vol. 377, Berlin:Springer-Verlag.

[35] A. A. Benzerga and J. Besson. Plastic potentials for anisotropic porous solids. Eur. J. Mech., 20(3):397-434, 2001.

[36] J. B. Stewart and O. Cazacu. Analytical yield criterion for an anisotropic material containing spherical voids and exhibiting tension-compression asymmetry. Int. J. Solids Struct., 48:357-373, 2011.

[37] R. A. Lebensohn, M. I. Idiart, P. Ponte Castaneda, and P.-G. Vincent. Dilatational viscoplasticity of polycrystalline solids with intergranular cavities. Philos. Mag., 91:30383067, 2011.

[38] S. M. Keralavarma and A. A. Benzerga. An approximate yield criterion for anisotropic porous media. C. R. Mecanique, 336:685-692, 2008.

[39] S. M. Keralavarma and A. A. Benzerga. A constitutive model for plastically anisotropic solids with non-spherical voids. J. Mech. Phys. Solids, 58:874-901, 2010.

[40] V. Monchiet, O. Cazacu, E. Charkaluk, and D. Kondo. Macroscopic yield criteria for plastic anisotropic materials containing spheroidal voids. Int. J. Plasticity, 24:1158$1189,2008$.

[41] D. Peirce, C. F. Shih, and A. Needleman. A tangent modulus method for rate dependent solids. Computers and Structures, 18:875-887, 1984.

[42] A. A. Benzerga, J. Besson, R. Batisse, and A. Pineau. Synergistic effects of plastic anisotropy and void coalescence on fracture mode in plane strain. Modell. Simul. Mater. Sci. Engrg., 10:73-102, 2002. 
[43] A. A. Benzerga, J. Besson, and A. Pineau. Anisotropic ductile fracture. Part II: theory. Acta Mater., 52:4639-4650, 2004.

[44] N. Aravas and P. Ponte Castaneda. Numerical methods for porous metals with deformation-induced anisotropy. Comput. Methods Appl. Mech. Engrg., 193:3767-3805, 2004 .

[45] R. Hill. A theory of yielding and plastic flow of anisotropic solids. Proc. Roy. Soc. London A, 193(1033):281-297, 1948.

[46] M. Kailasam and P. Ponte Castaneda. A general constitutive theory for linear and nonlinear particulate media with microstructure evolution. J. Mech. Phys. Solids, 46(3):427-465, 1998.

[47] J. Eshelby. The determination of the elastic field of an ellipsoidal inclusion, and related problems. Proc. Roy. Soc. London A, 241:357-396, 1957.

[48] K. Madou, J.-B. Leblond, and L. Morin. Numerical studies of porous ductile materials containing arbitrary ellipsoidal voids - II: Evolution of the length and orientation of the void axes. Eur. J. Mech., 42:490-507, 2013.

[49] M. Agoras and P. Ponte Castaeda. Iterated linear comparison bounds for viscoplastic porous materials with ellipsoidal microstructures. J. Mech. Phys. Solids, 61:701-725, 2013.

[50] A. C. Gavazzi and D. C. Lagoudas. On the numerical evaluation of Eshelby's tensor and its application to elastoplastic fibrous composites. Comput. Mech., 7:13-19, 1990.

[51] T. J. R. Hughes and J. Winget. Finite rotation effects in numerical integration of rate constitutive equations arising in large-deformation analysis. Int. J. Numer. Meths. Engrg., 15:1862-1867, 1980.

[52] S. Kweon and A. A. Benzerga. Finite element implementation of a macromolecular viscoplastic polymer model. Int. J. Numer. Meths. Engrg., 94:895-919, 2013.

[53] F. Scheyvaerts, P. R. Onck, C. Tekoglu, and T. Pardoen. The growth and coalescence of ellipsoidal voids in plane strain under combined shear and tension. J. Mech. Phys. Solids, 59:373-397, 2011.

[54] V. Tvergaard. Effect of stress-state and spacing on voids in a shear-field. Int. J. Solids Struct., 49:3047-3054, 2012.

[55] K. L. Nielsen, J. Dahl, and V. Tvergaard. Collapse and coalescence of spherical voids subject to intense shearing: studied in full 3D. Int. J. Frac., 177:97-108, 2012.

[56] T. Pardoen and J. W. Hutchinson. An extended model for void growth and coalescence. J. Mech. Phys. Solids, 48:2467-2512, 2000.

[57] S. M. Keralavarma, S. Hoelscher, and A. A. Benzerga. Void growth and coalescence in anisotropic plastic solids. Int. J. Solids Struct., 48:1696-1710, 2011. 
[58] K. Madou and J.-B. Leblond. A Gurson-type criterion for porous ductile solids containing arbitrary ellipsoidal voids - I: Limit-analysis of some representative cell. J. Mech. Phys. Solids, 60:1020-1036, 2012.

[59] K. Madou and J.-B. Leblond. A Gurson-type criterion for porous ductile solids containing arbitrary ellipsoidal voids - II: Determination of yield criterion parameters. $J$. Mech. Phys. Solids, 60:1037-1058, 2012.

[60] D. Song, M. Agoras, and P. Ponte Castaneda. The evolution of pore shape and orientation in plastically deforming metals: Implications for macroscopic response and shear localization. Mech. Mater., 90:47-68, 2015.

[61] B. Selvarajou, B. Kondori, A. A. Benzerga, and S. P. Joshi. On Plastic Flow in Notched Hexagonal Close Packed Single Crystals. J. Mech. Phys. Solids, 94:273-297, 2016.

[62] S. Kweon. Investigation of shear damage considering the evolution of anisotropy. $J$. Mech. Phys. Solids, 61:2605 - 2624, 2013.

[63] S. M. Keralavarma and A. A. Benzerga. Numerical assessment of an anisotropic porous metal plasticity model. Mech. Mater., 90:212-228, 2015.

[64] M. Gologanu. Etude de quelques problèmes de rupture ductile des métaux. PhD thesis, Université Paris 6, 1997.

[65] A. A. Benzerga, J. Besson, and A. Pineau. Anisotropic ductile fracture. Part I: experiments. Acta Mater., 52:4623-4638, 2004.

[66] C. Chu and A. Needleman. Void nucleation effects in biaxially stretched sheets. J. Engrg. Mat. Tech., 102:249-256, 1980.

[67] A. A. Benzerga. Micromechanics of coalescence in ductile fracture. J. Mech. Phys. Solids, 50:1331-1362, 2002.

[68] A. A. Benzerga and J.-B. Leblond. Effective Yield Criterion Accounting for Microvoid Coalescence. J. Appl. Mech., 81:031009, 2014.

[69] L. Morin, J.-B. Leblond, and A. A. Benzerga. Coalescence of voids by internal necking: theoretical estimates and numerical results. J. Mech. Phys. Solids, 75:140-158, 2015.

[70] M. E. Torki, A. A. Benzerga, and J.-B. Leblond. On Void Coalescence under Combined Tension and Shear. J. Appl. Mech., 82(7):071005, 2015. 